\title{
Predicting a detailed permeability profile from minipermeameter measurements and well log data
}

\author{
Shawn D. Nines \\ West Virginia University
}

Follow this and additional works at: https://researchrepository.wvu.edu/etd

\section{Recommended Citation}

Nines, Shawn D., "Predicting a detailed permeability profile from minipermeameter measurements and well log data" (2000). Graduate Theses, Dissertations, and Problem Reports. 1084.

https://researchrepository.wvu.edu/etd/1084

This Thesis is protected by copyright and/or related rights. It has been brought to you by the The Research Repository @ WVU with permission from the rights-holder(s). You are free to use this Thesis in any way that is permitted by the copyright and related rights legislation that applies to your use. For other uses you must obtain permission from the rights-holder(s) directly, unless additional rights are indicated by a Creative Commons license in the record and/ or on the work itself. This Thesis has been accepted for inclusion in WVU Graduate Theses, Dissertations, and Problem Reports collection by an authorized administrator of The Research Repository @ WVU. For more information, please contact researchrepository@mail.wvu.edu. 
Predicting a Detailed Permeability Profile from

Minipermeameter Measurements and Well Log Data

\author{
Shawn D. Nines
}

Thesis Submitted to the Faculty of the College of Engineering and Mineral Resources

West Virginia University

In Partial Fulfillment of the

Requirements for the Degree of

Master of Science

In

Petroleum and Natural Gas Engineering

Khashayar Aminian, Ph.D., PNGE, Chair

Sam Ameri, M.S., PNGE

H. Ilkin Bilgesu, Ph.D., PNGE

Daniel Della-Giustina, Ph.D., IMSE

Department of Petroleum and Natural Gas Engineering

Morgantown, West Virginia

2000 


\title{
Abstract \\ Predicting a Detailed Permeability Profile from Minipermeameter Measurements and Well Log Data
}

\author{
Shawn D. Nines
}

Permeability, along with the porosity, comprises one of the two most important properties in petroleum engineering with respect producing hydrocarbon fluids.

It is standard practice in the petroleum industry to determine permeability in one of two ways. These are pressure transient testing and core analysis. Both methods are expensive in their own ways. This research focuses on a way to minimize or limit the need for both of these testing procedures.

The purpose of this research was to utilize well log data, mainly gamma ray and density, Minipermeameter values, and basic information such as depth and spatial coordinates to predict permeability in the selected pilot area of the Stringtown field. This differed from previous research in that the known permeabilities of the cored wells were obtained using a Minipermeameter versus using only traditional core analysis.

This problem's solution may be in the utilization of Artificial Neural Networks. Recent studies have shown that permeability may be determined using ANNs and data obtained from wells logs, regardless of the heterogeneity of the reservoir. Log data, which has been shown to be prudent includes, gamma ray, density, and spontaneous potential. 


\section{Dedication:}

-This thesis is dedicated to my family, and especially to my late grandfather Hoza Bolyard, and late grandmother Gladys Nines.

\section{Acknowledgements:}

-Khashayar Aminian, Ph.D., PNGE, Chair

For his guidance and help throughout my graduate and undergraduate careers, especially with this thesis.

\section{-H. Ilkin Bilgesu, Ph.D., PNGE}

For his help with this thesis and for the knowledge I have gained in assisting him with various labs.

\section{-Sam Ameri, M.S., PNGE}

For his invaluable help and support from my first semester as a freshman to my last as a graduate student.

-Daniel Della-Giustina, Ph.D., IMSE

For his insight and help toward the completion of this thesis.

\section{-Beverly Matheny}

For her many hours of assistance and help for the past several years.

\section{-David and Mary Nines}

For the constant love, support, and encouragement that they have shown me throughout my entire life. 
Chapter One: Introduction 1

Chapter Two: Background and Theory 2

2.1 Stringtown Field 2

2.2 Permeability 5

2.3 A Brief Log Data Description $\quad 8$

$\begin{array}{ll}2.4 \text { Artificial Neural Networks } & 9\end{array}$

2.4.1 General Information 9

$\begin{array}{ll}\text { 2.4.2 Components } & 10\end{array}$

$\begin{array}{ll}\text { 2.4.3 Architectures } & 11\end{array}$

2.4.4 Activation Functions $\quad 15$

$\begin{array}{ll}\text { 2.4.5 Training } & 17\end{array}$

$\begin{array}{ll}\text { 2.4.6 Testing (Verification) } & 19\end{array}$

$\begin{array}{ll}\text { Chapter Three: Methodology } & 21\end{array}$

$\begin{array}{ll}\text { 3.1 Data Collection } & 21\end{array}$

3.1.1 Core and Well Log Data $\quad 21$

3.1.2 Minipermeameter Values 23

3.2 Overview of Neural Network Developments 27

3.3 Neural Network One Development 29

3.4 Application of Neural Network One 35

3.5 Neural Network Two Development 36

Chapter Four: Results and Discussion 41

4.1 Results of Neural Network One 41

4.2 Neural Network One Additional Verification (Production Sets) 46

4.3 Results of Neural Network Two 49

4.4 Neural Network Two Additional Verification 53 
Chapter Five: Conclusions and Recommendations

5.2 Recommendations

Appendix I - Log Comparisons for Cored Wells

Appendix II - Minipermeameter Averages for each cored well

Appendix III - Patterns for NN One, NN Predicted and Actual

Appendix IV - NN One Verification Network with Cw1 as Production Set 79

Appendix V - NN One Verification Network with Cw2 as Production Set 81

Appendix VI - NN One Verification Network with Cw3 as Production Set 83

Appendix VII - NN One Verification Network with Cw4 as Production Set 85

Appendix VIII - NN One Verification Network with Cw5 as Production Set 87

Appendix IX - Patterns for NN Two, NN Predicted and Actual 89

Appendix X - NN Two Verification Network with Cw1 as Production Set 91

Appendix XI - NN Two Verification Network with Cw2 as Production Set 93

Appendix XII - NN Two Verification Network with Cw3 as Production Set 95

Appendix XIII - NN Two Verification Network with Cw4 as Production Set 97

Appendix XIV - NN Two Verification Network with Cw5 as Production Set 99

Appendix XV - Pilot Area Predictions of Permeability 101

References 


\section{List of Figures:}

Figure 1. The Location of the Springtown Field 2

Figure 2. Positions of Cored and Pilot Wells 4

$\begin{array}{lll}\text { Figure 3. } & \text { Schematic of Minipermeameter } & 7\end{array}$

$\begin{array}{lll}\text { Figure 4. A simple artificial neural network } & 11\end{array}$

$\begin{array}{lll}\text { Figure 5. Standard networks } & 12\end{array}$

Figure 6. Jump Connection Networks 12

$\begin{array}{llr}\text { Figure } 7 . & \text { Recurrent Network } & 13\end{array}$

$\begin{array}{lll}\text { Figure 8. Ward Network } & 13\end{array}$

$\begin{array}{lll}\text { Figure 9. Kohonen Network } & 14\end{array}$

$\begin{array}{lll}\text { Figure 10. General Regression Neural Network } & 14\end{array}$

Figure 11. Group Method of Data Handling or Polynomial Network 15

Figure 12. Schematic of the training of a Backpropagation neural network 18

Figure 13. Illustration of a Generic Function for Slope Determination 22

Figure 14. Minipermeameter Values vs. Core Permeability in Cw 1

Figure 15. Illustration of measuring procedure of Minipermeameter 25

Figure 16. Logging Tool Illustration 25

Figure 17. Actual Minipermeameter Values for Cw 1

Figure 18. Average Minipermeameter Values for Cw 1

Figure 19. Flow Chart of Thought Process in Solution 28

Figure 20. Preliminary Structure of Neural Network One 32

$\begin{array}{lll}\text { Figure 21. Final Structure of NN One } & 34\end{array}$

Figure 22. Spreadsheet used to generate core permeabilities from log data and $\begin{array}{ll}\text { Minipermeameter values } & 35\end{array}$

Figure 23. Preliminary Structure of NN Two 38

Figure 24. Final Structure of NN Two 40 
Figure 25. Actual \& NN Predicted for NN One Applied to Pattern Set 43

Figure 26. Actual vs. NN Predicted for NN One Applied to Pattern Set 43

Figure 27. Actual vs. NN Predicted for NN One Applied to Test Set 44

Figure 28. Actual vs. NN Predicted for NN One Applied to Training Set 44

Figure 29. Cw1 used as a Production Set, Pattern Set 48

Figure 30. Cw1 used as a Production Set, Test Set 48

Figure 31. Cw1 used as a Production Set, Production Set $\quad 49$

Figure 32. Actual \& NN Predicted for NN Two Applied to Cw 1 Pattern Set 50

Figure 33. Actual vs. NN Predicted for NN Two Applied to Cw 1 Pattern Set 51

Figure 34. Actual vs. NN Predicted for NN Two Applied to Cw 1 Test Set 51

Figure 35. Actual vs. NN Predicted for NN Two Applied to Cw 1 Training Set 52

Figure 36. Cw1 used as a Production Set, Pattern Set 56

Figure 37. Cw1 used as a Production Set, Test Set 56

Figure 38. Cw1 used as a Production Set, Production Set $\quad 57$

$\begin{array}{lll}\text { Figure } 39 . \quad \text { Location of the Pilot Wells } & 58\end{array}$

Figure 40. NN Predicted Permeability of Pilot Well 1

Figure 41. NN Predicted Permeability of Pilot Well 2

Figure 42. NN Predicted Permeability of Pilot Well $3 \quad 60$

Figure 43. NN Predicted Permeability of Pilot Well 4

Figure 44. NN Predicted Permeability of Pilot Well 5

Figure 45. NN Predicted Permeability of Pilot Well 6

Figure 46. NN Predicted Permeability of Pilot Well 7 62 


\section{List of Tables:}

Table 1. Contribution values of inputs in NN One

Table 2. R Squared Values for the Verification Networks of NN One

Table 3. Contribution Values of Inputs in NN Two 


\section{Nomenclature}

$\begin{array}{ll}\text { ANN: } & \text { Artificial Neural Networks } \\ \text { Cw1: } & \text { Cored Well \# } 1 \\ \text { Cw2: } & \text { Cored Well \# } 2 \\ \text { Cw3: } & \text { Cored Well \# } 3 \\ \text { Cw4: } & \text { Cored Well \# } 4 \\ \text { Cw5: } & \text { Cored Well \# } 5 \\ \text { GR: } & \text { Gamma Ray Log } \\ \mathrm{ft}: & \text { feet (length) } \\ \text { k: } & \text { Permeability } \\ \text { mD: } & \text { millidarcies }\end{array}$

mini-permeability: permeability as seen by the Minipermeameter

PI1: $\quad$ Pilot Well \# 1

PI2: $\quad$ Pilot Well \# 2

PI3: $\quad$ Pilot Well \# 3

PI4: $\quad$ Pilot Well \# 4

Pl5: $\quad$ Pilot Well \# 5

PI6: $\quad$ Pilot Well \# 6

PI7: $\quad$ Pilot Well \# 7

$\mathrm{R}^{2}: \quad$ Correlation Coefficient

RHOB: $\quad$ Density Log 


\section{Chapter One Introduction:}

Permeability and porosity are two of the most important properties in petroleum engineering when attempting to produce hydrocarbon fluids in an effective and efficient way. Low values in permeability and/or porosity can be the lone deciding factor(s) in the method or lack of exploitation in a field. Permeability is defined as the interconnection between pores and determines how fluid travels throughout a rock. In general, the greater the permeability, the greater the possible fluid flow throughout the rock.

Commonly, permeability is determined in one of two ways, either pressure transient testing or core analysis. Both methods are expensive in their own ways. This research focuses on a way to minimize or limit the need for both of these testing procedures.

The purpose of this research was to utilize well log data, mainly gamma ray and density, minipermeameter values, and basic information such as depth and spatial coordinates to predict permeability in the selected pilot area of the Stringtown field. The results of this study can be used with a reservoir simulator to help map reservoir permeability for the entire field. This differed from previous research in that the known permeabilities of the cored wells were obtained using a minipermeameter versus using only traditional core analysis.

This problem's solution may be in the utilization of Artificial Neural Networks. Recent studies have shown that permeability may be determined using ANNs and data obtained from wells logs, regardless of the heterogeneity of the reservoir. Log data, which has been shown to be prudent includes, gamma ray, density, and spontaneous potential. ${ }^{1,2,3,4}$ 


\section{Chapter 2 Background and Theory}

\subsection{The Stringtown field:}

Located in northwestern West Virginia, the Stringtown oilfield is situated on the tri-county borders of Tyler, Wetzel, and Doddridge counties. The producing formation has historically, and continues to be the Upper Devonian Gordon Sandstone. Geological studies of this formation have concluded its birth to be consistent with deposits from a shallow marine shoreline. The Upper Devonian Gordon Sandstone is comprised of a sandstone with relatively thin shale and conglomerate layers. The thickness generally ranges from a minimum of 10 feet to the maximum of 25 feet, occurring in the Northeast and Southwest trends of the field. The formation is usually encountered at a depth of approximately 2950 feet. With a length of about five miles $(8.05 \mathrm{~km})$ and width of $2 \frac{1}{2}$ miles $(4.02 \mathrm{~km})$, the production area is estimated at 8900 acres.

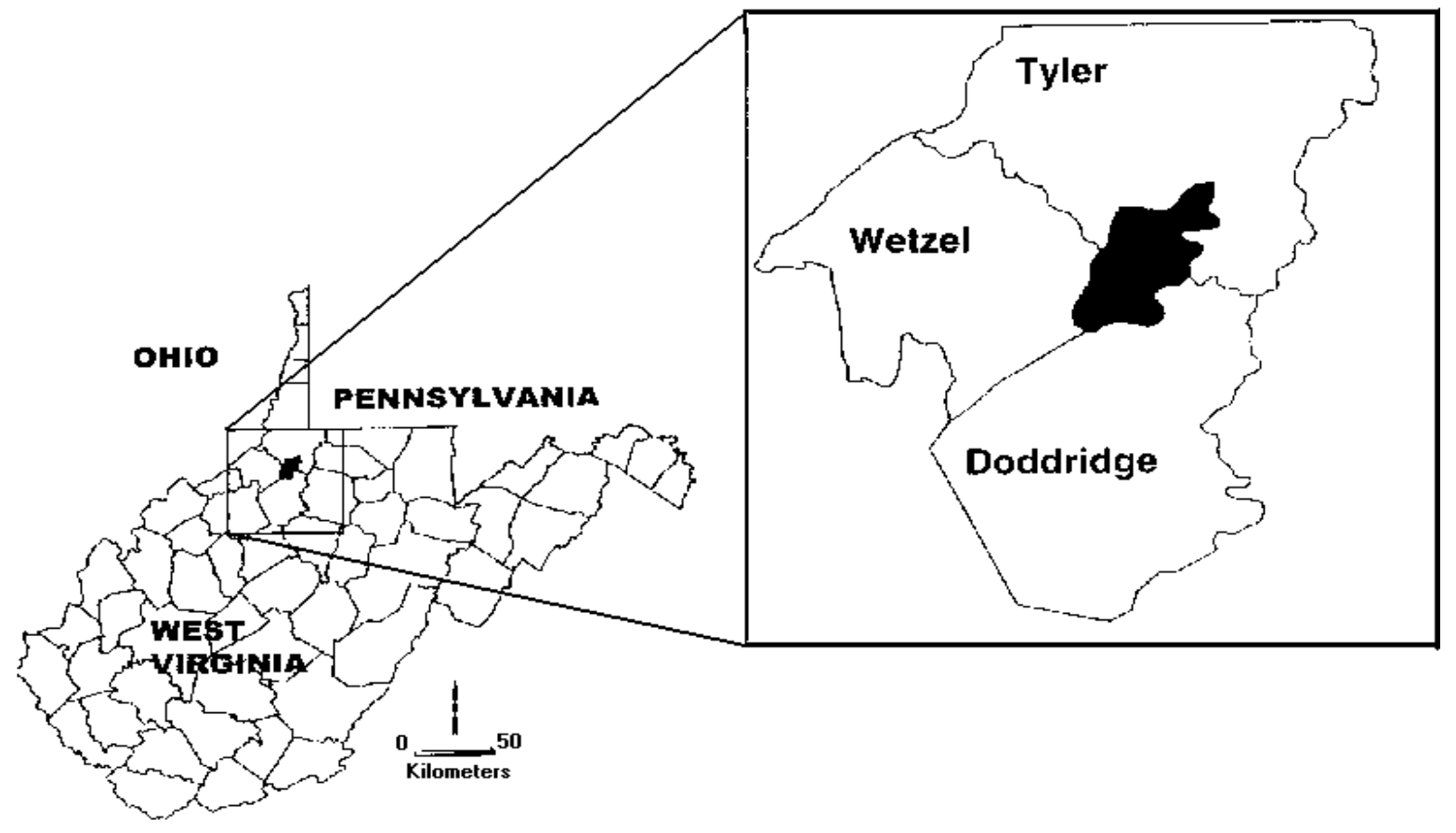

Figure 1. The Location of the Stringtown Field 
Cumulative oil production throughout the life of the field has been around 13 million barrels, of the estimated 88.5 million barrels of oil initially in place. Oil produced from the reservoir has an API gravity of 44 degrees (@66 degrees Fahrenheit) and a viscosity of $3.5 \mathrm{cp}$. at atmospheric pressure (14.7 psi) and a temperature of 75 degrees Fahrenheit.

The Stringtown field began its production history around 1890. From that point and until 1901, around five hundred wells were drilled. This period of exploitation only progressed to 1910 . By 1910 , most of the original 500 wells had been plugged due to low primary recovery and paraffin problems. In the 1940s, the field was chosen for a gas recycling project, again the results were less than satisfactory.

Beginning in 1980, the Stringtown field was the location of a waterflooding trial project. This dual-five-spot waterflood, covering 33 acres, continued with successful results until 1985. Based on these results, a complete waterflooding project was initiated, with the drilling of one hundred water injection wells and 40 production wells. The field was divided into three units of development. Unit 1 is located in the middle of the field and covers 1815 acres. This unit is also the location of the original waterflood trial. Unit 2 covers 5723 acres and is located in the north of the development area. Unit 3 covers 1360 acres and is located in the south of the development area. Several wells in these units have been cored and are indicated on figure 2 . It is important to note that an estimated 1.8 million barrels of oil have been produced due to this secondary recovery. ${ }^{5,6,7}$ 


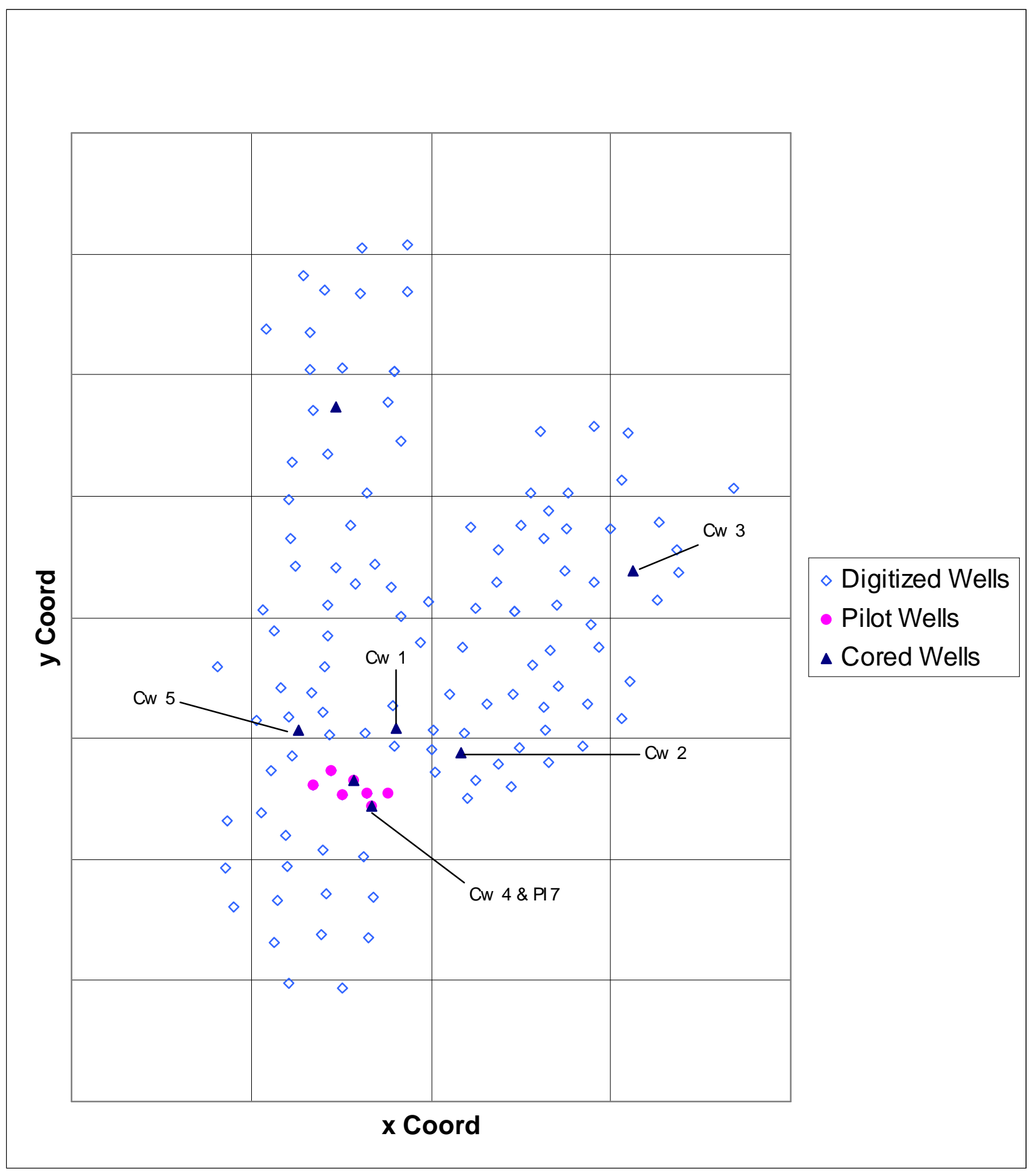

Figure 2. Positions of Cored and Pilot Wells 


\subsection{Permeability:}

Permeability, along with the porosity, comprises one of the two most important properties in petroleum engineering with respect to effectively and efficiently producing hydrocarbon fluids. Lack a permeability and/or porosity may be the lone deciding factor(s) in the method or lack of exploitation in the field. Permeability, usually depicted by the variable"k" and measured in millidarcies $(\mathrm{mD})$, is generally defined as the interconnection between pores within a hydrocarbon bearing rock. This rock property was originally defined in 1856, by Darcey and expressed as an independent variable in his equation:

$$
\mathrm{V}=\mathrm{k} / \mu(\mathrm{dp} / \mathrm{dx})
$$

Permeability determines how fluid travels from pore to pore in the rock, and finally from pore to wellbore. The greater the permeability the greater the possible fluid flow throughout the rock. Factors which affect permeability, include grain size, grain shape, grain distribution, grain packing and cementation.

It is standard practice in the petroleum industry to determine permeability in one of two ways. These are pressure transient testing and core analysis. Each method has within it certain advantages and disadvantages.

Pressure transient testing has become an important tool for diagnostic purposes in the petroleum industry. This testing is done by gathering pressure data recorded at specific times and specific flow rates. The pressure data is analyzed using specialized plots and/or matching the resulting data to type curves. These tests can provide valuable information such as average permeability, wellbore damage (skin + or -), drainage volume and natural reservoir pressure. The two most commonly used forms of 
pressure transient tests are drawdown tests, and build-up tests. Unfortunately, both require that the well be shut-in for an extended period of time, which results in a loss of production and revenue. Also, a well test may yield results which can lead to conflicting conclusions.

Core analysis allows the engineer a unique view into the formation of concern by allowing the actual rock itself to be tested for properties such as permeability and porosity. Coring is done by using a hollowed bit to drill through the zone of interest. This bit cuts a core from the formation, and this core is returned to the surface. The core may then be tested in a laboratory under reservoir conditions. Because of its excessive price, coring can only be performed only a few select wells in a reservoir. In order to effectively model the reservoir, these cored wells must be distributed in an orientation which will be representative of the reservoir and its major heterogeneous components.

In this study, a device called a minipermeameter was also used to measure permeability values. This device is an electro-pneumatic mechanism that is made up of a pressured tank of nitrogen, a flow meter, a permeability probe and finally a pressure sensor. The flow meter and pressure sensor are directly connected to a computer equipped with specialized software. The computer also serves to record the readings. The pressure sensor is designed to detect any incremental pressure changes and the flow velocity, while the probe is in contact with the core surface. When the pressure becomes stable and the flow becomes constant, the data is converted into permeability values and recorded by the computer. A schematic of this is in figure 3 . 


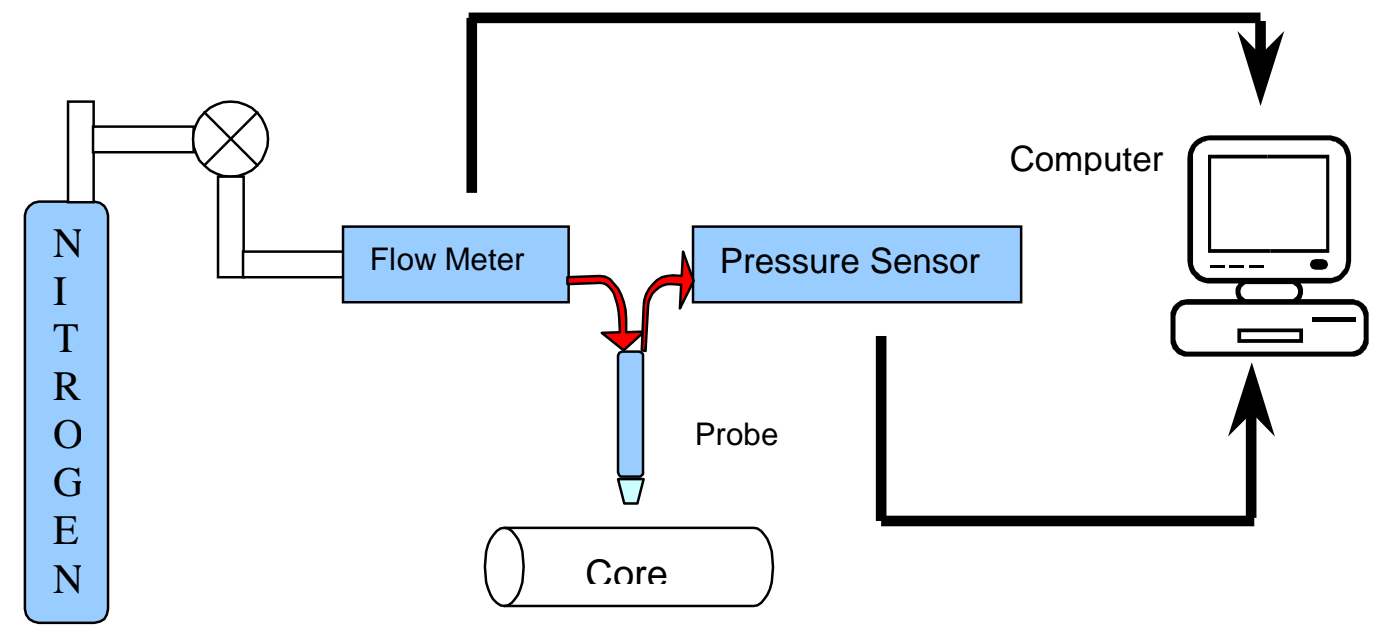

Figure 3. Schematic of Minipermeameter

For many years engineers have tried to determine a relationship between porosity, density, and permeability, although a general relationship has proven to be elusive. This would be very beneficial since porosity can easily be determined with the utilization of well logs, which are run on the majority of wells in any given in reservoir.

This problem's solution may be in the utilization of Artificial Neural Networks. Recent studies have shown that permeability may be determined using ANNs and data obtained from well logs, regardless of the heterogeneity of the reservoir. 


\subsection{A Brief Log Data Description:}

Two main logs were used in this study, Gamma Ray (ohm-m) and Density (gm/cc), sometimes called Bulk Density(RHOB). This was done for two reasons, first because of the theoretical relationships between them and permeability, and also these logs are available on most of the wells in any particular reservoir. The Gamma Ray measures the natural radioactivity of a formation, and thus can help to determine the lithology, especially with respect to shaleiness. Gamma rays themselves are electromagnetic radiation with wavelengths between $1.0 \times 10^{-9}$ and $1.0 \times 10^{-11} \mathrm{~cm}$. The Density Logging tool measures electron density, which is generally the same as bulk density, and its traditional usage is for the determination of porosity. Low densities correlate closely to high porosities. ${ }^{8}$ 


\subsection{Artificial Neural Networks:}

\subsubsection{General Information:}

Artificial neural networks have recently shown success in the solutions of problems and predictions of data to have a wide range of disciplines. Financial analysis and forecasting, medical research, help with managerial decisions, engineering problems with connected data, and even predictions about human behavior have benefited from artificial neural networks. These networks have additional advantages over direct problem solving and forecasting techniques (e.g. traditional algorithms and equations) in their ability to discover and utilize patterns and relationships within the known data set. Artificial neural networks can uncover patterns and/or relationships which have been previously unknown or that change from data set to data set. ${ }^{9,10,11}$

The origins of these networks have been drawn for the biology which precipitates learning in the human brain. An artificial neural network attempts to mimic this biology and process. The great advantage of this biology are its qualities of unrigidness and the ability to disseminate important data from unimportant data. The foundation of their

unique computing ability is the concept of pattern recognition. Just as our brains learn to solve problems by recognizing specific patterns and how they relate to specific outcomes, so do artificial neural networks. In general, artificial neural networks are parallel distributed information systems that utilize knowledge about how the human learning process works to recognize patterns, learn from those patterns, and memorize patterns which produce the desired outcomes.

Pattern recognition is explained as the mapping within a pattern category into a class category. In other words pattern recognition is the process in which unique classes are identified for sets of features, or inputs, which contribute significantly to the 
output. This is done without confusion from variations, omissions, or occasionally unrepresentative data. The overall purpose is to define meaningful relationships between the information available and the output of concern. Artificial neural networks attempt to manipulate raw data into useful representative symbols from which knowledge can be obtained. This procedure often produces connections which are too obscured within the data to be obvious. It is these characteristics that make these networks promising in petroleum engineering. ${ }^{5}$

\subsubsection{Components ${ }^{10}$ :}

Artificial neural networks can be divided into several different components. The first and most basic is the neuron. Neurons are the processors that are allocated to execute the most simple tasks. When information is passed to the neuron, the neuron processes this information by applying a mathematical activation function to it and then producing a new output signal. These activation functions are generally non-linear in nature. Each neuron is connected to other neurons by connection links that allow for the transfer of data. Connection links have an associated weight that determines how influential their data is to the network.

Neurons are generally grouped together to create slabs, and slabs are grouped together to create layers. A general artificial neural network has three layers of slabs, one input layer, one middle layer, and one output layer. The input layer is used to present data to the network, as its purpose is to retrieve information and send that information to the middle layer. Every neuron in the input layer is connected to every neuron in the middle layer. This allows the data to be processed simultaneously. Also, the number of neurons in this layer is generally determined by the number of inputs. 
The middle layer is considered to be the actual computational hub of the artificial neural network. It is responsible for the analysis of information presented and is where the pattern recognition takes place. This layer receives data, organizes and transforms it into a simple symbolic form from which the network can more readily learn. Figure 4 is an illustration of a simple artificial neural network which demonstrates these connections.

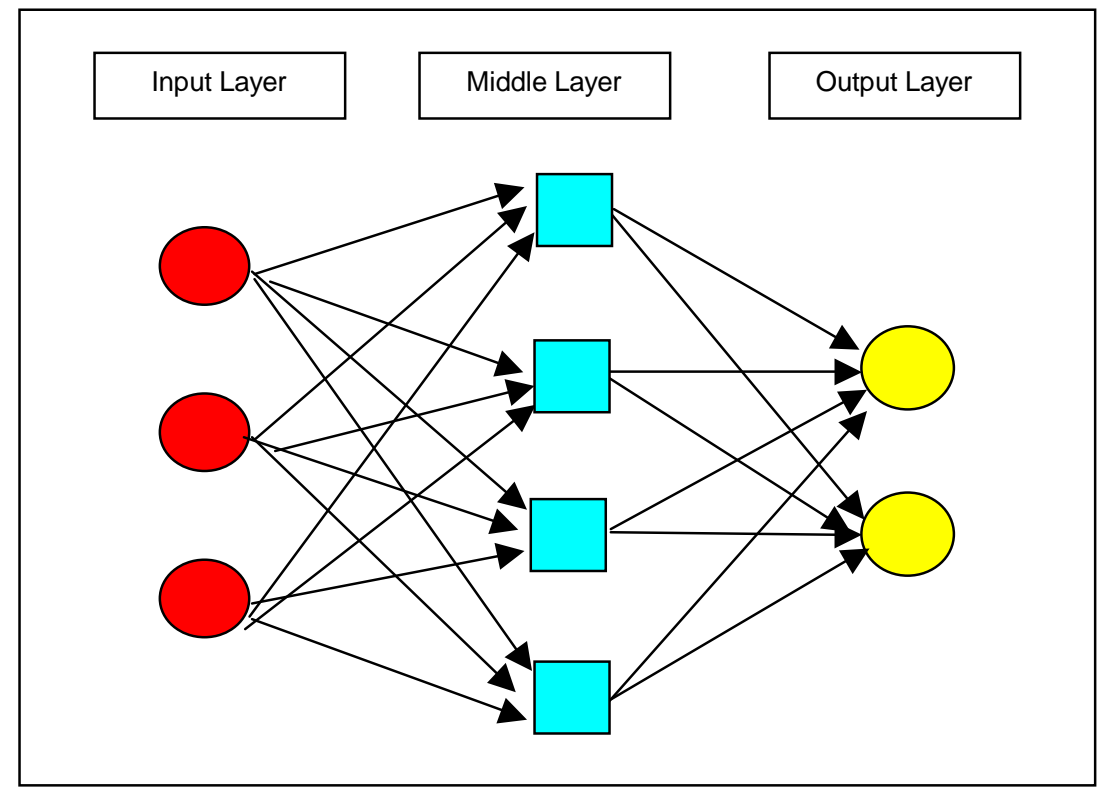

Figure 4. A simple artificial neural network

\subsubsection{Architecture:}

The architecture of an artificial neural network is defined as the order, number, and direction of its connections. Architecture determines the amount of knowledge a network can store, and the algorithms used in the weight updates that are the backbone of learning. Many architectures are available and each one provides its own benefits. 
Back-propagation models seem to stand out as the most widely used networks in recent years. These architectures are known for their prediction abilities, and good generalization on an extended variety of problems. Back-propagation nets are considered supervised, which means they are trained by being exposed to both inputs and target outputs. Within this category there are several variations, and they are as follows:

Standard Networks are characterized as having each layer connected to the immediately preceding layer.

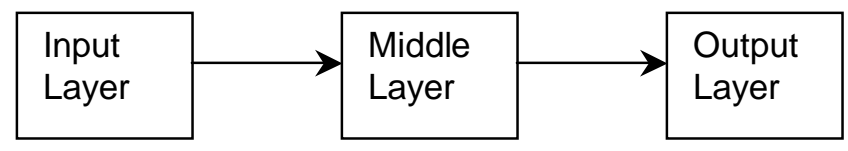

Figure 5. Standard networks

Jump Connection networks are characterized as having each layer connected to every previous layer.

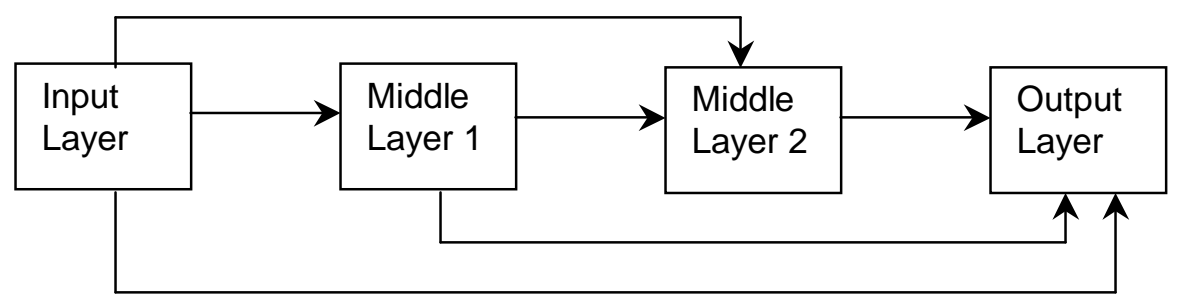

Figure 6. Jump Connection Networks 
Recurrent networks are characterized with dampened feedback from any layer, input, middle, or output.

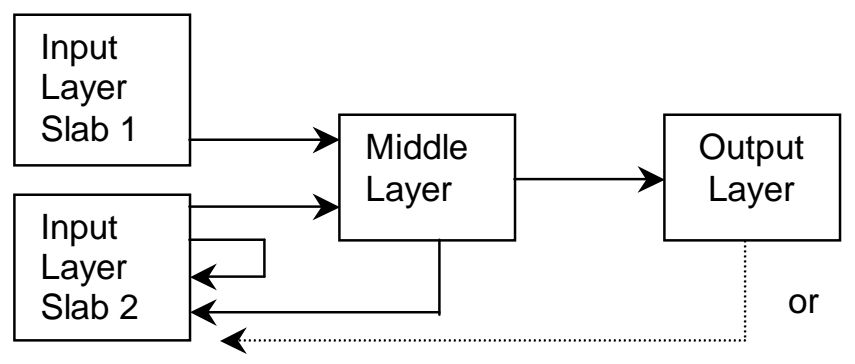

Figure 7. Recurrent Network

Ward networks are characterized by multiple slabs in the middle layer. Ward networks are very useful in their computing capacities when each Middle slab is given a different activation function. Ward networks are the default chosen by the neural network construction program used.

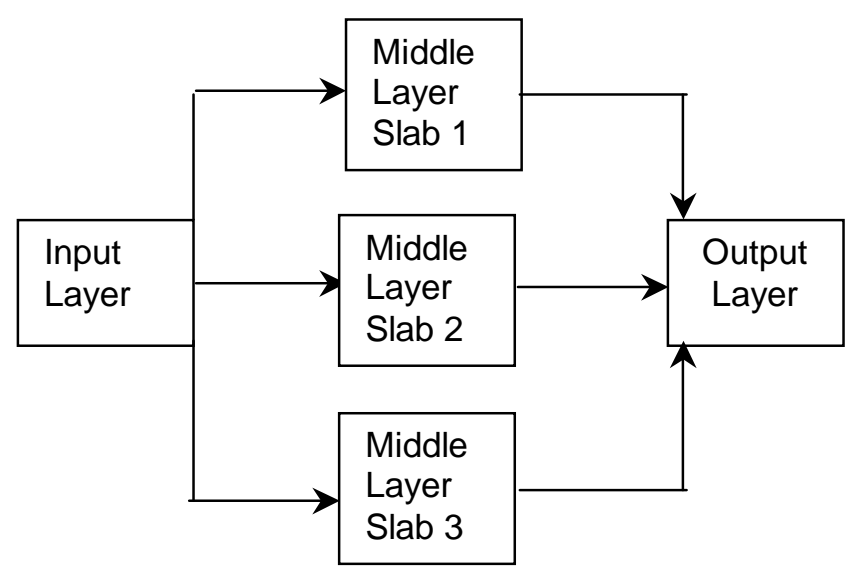

Figure 8. Ward Network 
The Kohonen Self Organizing Map is characterized as an unsupervised network. The architecture only has two layers, input and output. This network can learn without being exposed to any selected target outputs. They are used mainly to store data into specific classes.

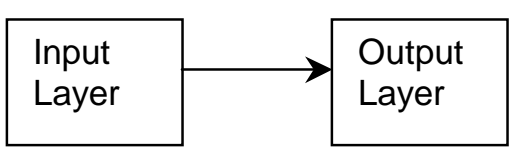

Figure 9. Kohonen Network

Probabilistic Neural Networks are also a supervised net known for their prediction capabilities. They have the additional benefit of organizing data into a specified number of output categories.

General Regression Neural Networks can train quickly on sparse data but, instead of categorizing that data, General Regression Networks produce continuous valued outputs. These networks evaluate each output independently of all other outputs, this may make them useful when multiple outputs are desired.

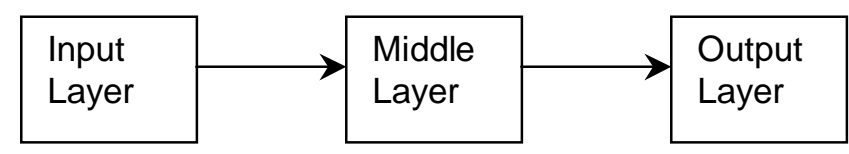

Figure 10. General Regression Neural Network 
Group Method of Data Handling or Polynomial Networks work by building successive layers connected by lengths that are simple polynomial terms. The beginning layer is the input layer. The first layer to be created is made by computing the regressions of the input variables and choosing the best ones. The second layer is created by finding the same regressions from the first layer and the input variables at which time the network chooses the best with the survivors. This process continues until the network stops discovering any substantial improvement. These networks are known for their abilities to prevent over learning problems.

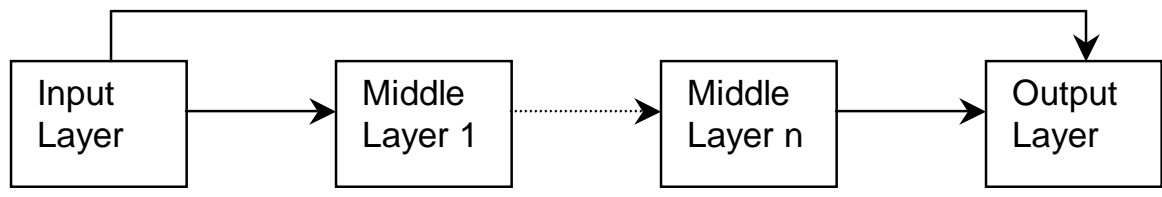

Figure 11. .Group Method of Data Handling or Polynomial Network

\subsubsection{Activation Functions}

Activation functions are the basic operation of the neuron as it receives a weighted input signal and produces an output signal to be sent to the next layer. Each activation function has a potential of viewing the data set in different ways. Linear, Binary, Sigmoid, and Probabilistic are the four categories in which each activation function may be placed. A brief description of some of the functions follows. 
Linear functions are used primarily in the input layer causing the input data to be sent directly to the middle layer.

Identity: $f(x)=x$

Linear Scaled: $f(x)=m x+b$

Binary functions are used to convert continuous data into a more useful binary unit. This is beneficial in classification networks.

Step: $f(x)=1$ if $x \geq b$ or $f(x)=0$ if $x<b$

Sigmoid functions are generally 'S" shaped curves and are very useful in the use of back propagation networks. This is because of the simple relationship between the value of the function at any point and the value of the derivative at any point, this characteristic reduces computational overburden during the training process.

Logistic: $f(x)=\frac{1}{\left(1+e^{-\sigma x}\right)}$

Hyperbolic tangent: $f(x)=\tanh (x)$

Hyperbolic tangent 1.5: $f(x)=\tanh (1.5 x)$

Symmetric logistic: $f(x)=\frac{2}{\left(1+e^{-\sigma x}\right)^{-1}}$ 
Probabilistic functions have the uniqueness of being non-increasing. The Gaussian function has the appearance of the classic bell shaped curve. It maps high values into low values, helping to bring out meaningful features within the mid ranged values. The Gaussian complement function does just the opposite bring out meaningful features at the extreme ends of the data set.

\subsubsection{Training:}

Artificial neural networks must undergo a training process in order to learn to solve the problem at hand. Training for each network depends on its architecture, but most have similarities to the back propagation model. Back propagation uses the generalized Delta rule. This is utilized in order to reduce the total squared error with respect to the output. Back propagation essentially means propagating or sending back error to the previous connection links between layers and adjusting the corresponding weights as needed. The optimum goal of this is to have a training that balances its ability to identify correctly the input patterns used in training, and also to give reasonable responses to similar input not used in the training.

There are three steps involved in the training of a back propagation network. These are the feedforward of the input training pattern, the calculation and back propagation of the error, and the adjustment of the weights.

The feedforward process begins with all weights associated to the connection links initialized. Information is provided to the network through the input layer. Input data is multiplied by the initial weights. The sum product of all input neurons and their corresponding weights are then transmitted to the middle layer and each neuron therein. Each of the middle neurons maps the sum to a new output signal using its specified 
activation function. This result is multiplied by the weights of the connection links between the middle at output neurons/neuron. The output neuron calculates the sum of the middle layer's weighted inputs to determine the actual output.

Once this has been done each output unit compares its predicted output with its target output, determining the associated error for that pattern with that specific unit. With this information, the network is able to back propagate this error. A correction factor is calculated with the generalized Delta Rule. These correction factors are sent back from each output neuron to all middle neurons. A similar process takes place as a second correction factor is sent from the middle neurons to the input neurons. Once all of the conversion factors have been determined the weights for all layers are adjusted at the same time. This adjustment is a function of the correspondent correction factor and activation function of the previous neuron. Figure 12 shows an illustration of this process.

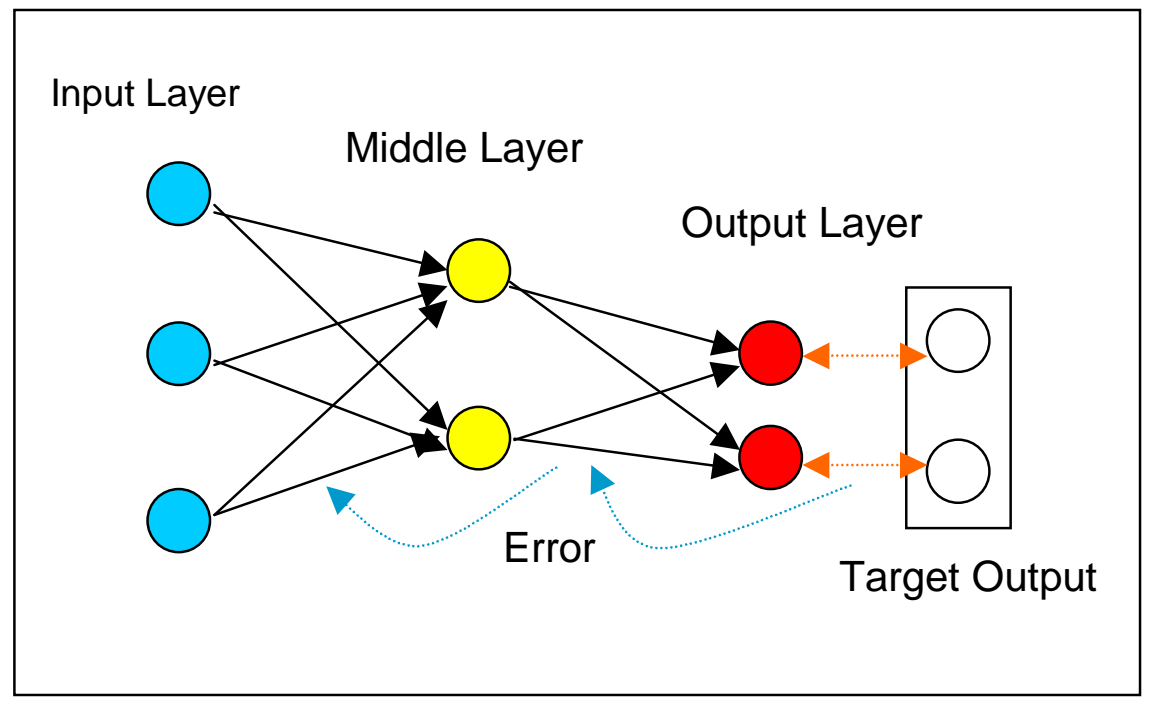

Figure 12. Schematic of the training of a Backpropagation neural network 
There are several values which must be set in order to control the training. The two major settings are learning rate and momentum. Together these two parameters determine the speed and stability of the learning.

Every time a pattern is exposed to the network, the weights preceding the output neuron are modified in the direction which appears to produce a smaller error. The property of learning rate controls the amount of change in the weights leading toward that smaller error. If the learning rate is high, the weight changes will be large, and learning will occur faster. Large learning rates have the disadvantage of often causing an oscillation of the weight changes. This will cause the model to diverge from the optimum conclusion. The property of momentum helps to prevent oscillation of weight changes and force the convergence by making the weight change a function of the previous weight change. This action provides a smoothing effect. The momentum factor determines what portion of the last weight change that is added in the new calculation.

\subsubsection{Testing (Verification):}

Is important for an artificial neural network to have a good balance between memorization and generalization. By allowing an artificial neural network to train too long, the balance is tipped toward the memorization side. The solution to this problem is introduction of two separate sets, a training set and a test set. The pattern set consists of both the test and training. Weight adjustments are applied based on the training set, but to prevent memorization, the network does not constantly see the test set. During predefined intervals during training, the error is computed using the test set. Calibration is the way these predefined intervals are selected. The calibration interval is the number of training events which take place before the network is compared to the test set. These 
provide a way of preventing memorization by the network. The network is usually saved on the minimum error of this test set, rather than the training set.

The artificial neural network is then considered to have reliable results. This allows for the application of the network to new never before seen data sets. These data sets must consist of the same inputs, but the output values will determined based on the previously saved minimum test set error network. 


\section{Chapter Three Methodology:}

\subsection{Data Collection:}

\subsubsection{Core and Well Log Data:}

The initial step in the solution to this problem was to collect the appropriate data. For the study to be credible, data must be available in or very near the area of concern, which in this case was the original pilot injection area of the West Virginia Stringtown Field. The results of this research would only be representative of that specific location, because of the constantly changing reservoir properties. These properties become more pronounced as one moves farther away from the pilot area. Core data for several wells which met the required criteria were available, and also minipermeameter values for as many wells was accessible. The usable data although, was severely limited because, as previously stated, only wells with both core analysis and minipermeameter readings would be beneficial. The requirement to have both of these qualities reduced this set to six cored wells. Also, the extreme differences in properties of Cored Well Number Six excluded one more well, leaving only five.

The next essential data required and gathered were the actual logs of these wells. To use this log data within the confines of artificial neural networks it also had to be digitized and adjusted to correspond correctly, with respect to depth, to the minipermeameter values and core data. Both of these concerns were addressed and corrections were made in previous studies. ${ }^{3}$

It is important to note that the actual digitized logs only had readings for gamma ray(ohm-m) and density(g/cc) not the slopes(first derivatives). These values were calculated. The slopes would prove to be useful to the networks by indicating rates of change with respect to depth in the log data. The slopes were calculated using a threepoint method. This method computes the derivative at a specific point as a function of 
the weighted average of the preceding and following slopes relative to that point. An illustration of this is shown in Figure 13.

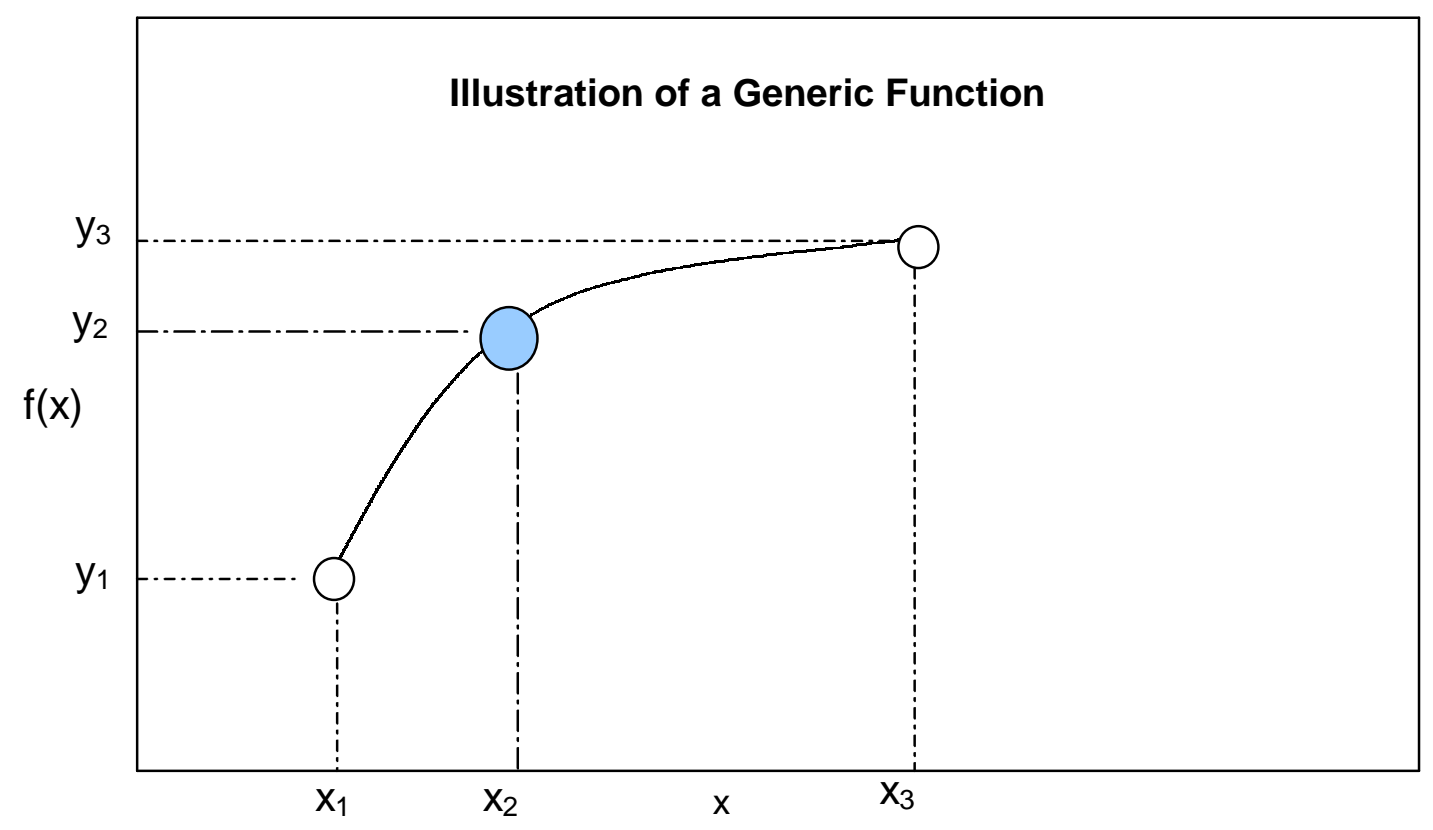

Figure 13. Illustration of a Generic Function for Slope Determination

Referring to Figure 13 the slope of point two, denoted as $m_{2}$ is calculated by:

$$
\begin{aligned}
& \mathrm{m}_{\mathrm{l}}=\frac{\mathrm{y}_{2}-\mathrm{y}_{1}}{\mathrm{x}_{2}-\mathrm{x}_{1}} \\
& \mathrm{~m}_{\mathrm{r}}=\frac{\mathrm{y}_{3}-\mathrm{y}_{2}}{\mathrm{x}_{3}-\mathrm{x}_{2}} \\
& \mathrm{~m}_{2}=\frac{\left(\mathrm{x}_{3}-\mathrm{x}_{2}\right) \mathrm{m}_{1}+\left(\mathrm{x}_{2}-\mathrm{x}_{1}\right) \mathrm{m}_{\mathrm{r}}}{\left(\mathrm{x}_{3}-\mathrm{x}_{2}\right)+\left(\mathrm{x}_{2}-\mathrm{x}_{1}\right)}
\end{aligned}
$$

The final two data values in the log information were the gamma ray and density baselines. The importance of these values are in their ability to define information to the 
networks on tool calibration during the logging procedure. Additionally, the gamma ray baseline provides a view of the shaleiness of the surrounding formations. These values were determined through the trending of each log.

The core data collected included basic data such as spatial coordinates and depth, which provide information about how the reservoir changes based on location. The remaining core data collected was the permeability value, in millidarcies, for each tested depth.

The total log and core data collected is as follows:

$\begin{array}{ll}\text {-Gamma Ray(GR) } & \text {-Gamma Ray Baseline(GR BL) } \\ \text {-Density(RHOB) } & \text {-Density Baseline(RHOB BL) } \\ \text {-Gamma Ray Slope(GR Slope) } & - \text { X coordinate } \\ \text {-Density Slope(D Slope) } & - \text { Y coordinate } \\ \text {-Depth } & \end{array}$

\subsubsection{Minipermeameter Values:}

The last data collected were the permeability values measured by the minipermeameter. These raw values were collected for the wells corresponding to the core data. The minipermeameter values differ from the core permeability values in two ways. The first is that the minipermeameter values are significantly lower. The second is that the mini-permeability is more sporadic in nature. (Figure 14). 


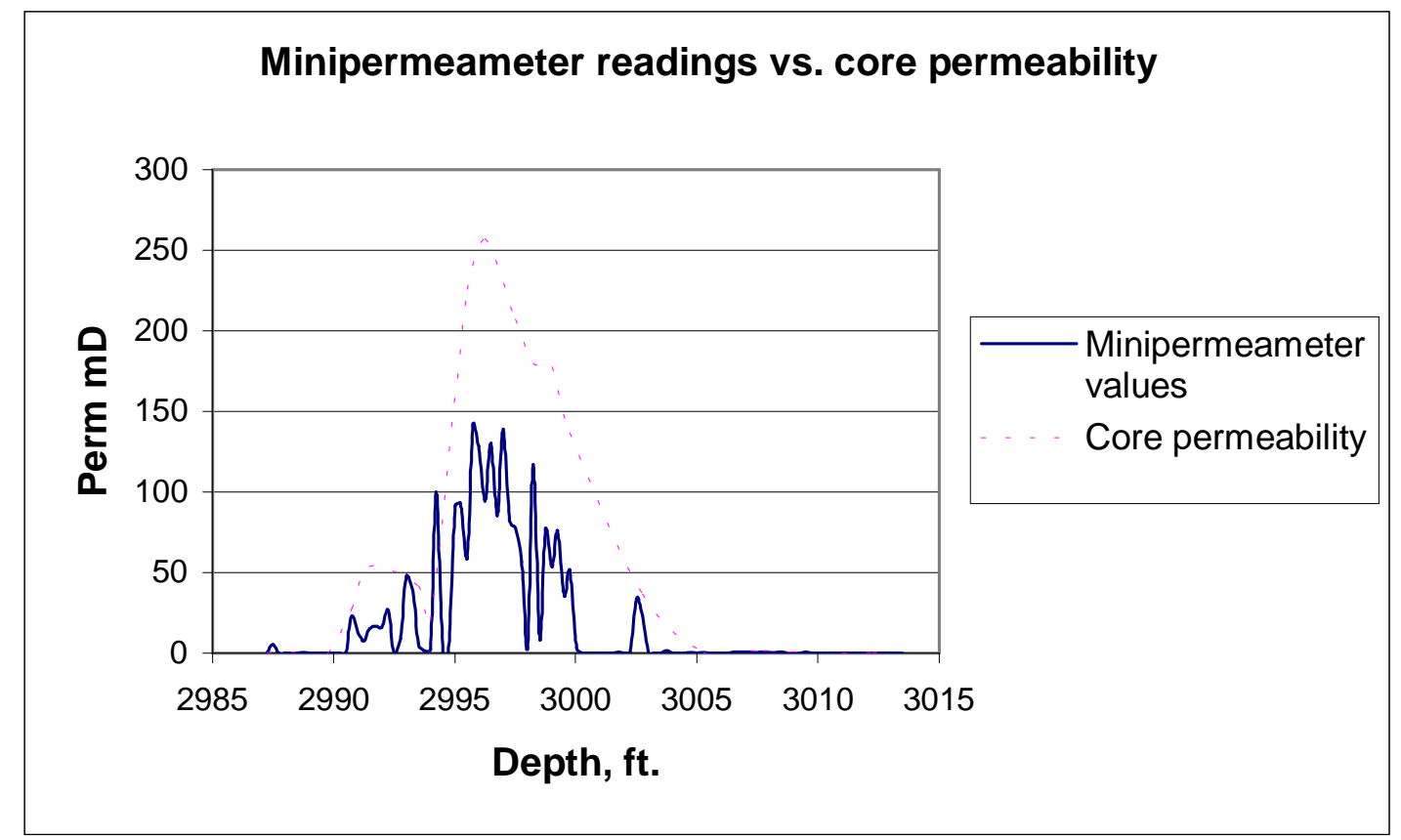

Figure 14. Minipermeameter Values vs. Core Permeability in Cw 1

The differences in values are caused by a collection of factors including methods of measurements, depth of investigation, and possible deterioration of the cores over time. These factors will be discussed later. The sporadic nature of the minipermeameter values is caused by the method of measurement and had to be corrected to correlate with the core permeability values, measured at one foot intervals, and the log data.

The traditional core data was obtained over a larger core section, usually about one foot; whereas the minipermeameter was taken at quarter foot intervals. This characteristic difference causes a much more sporadic data set in the minipermeameter when compared to the smooth average-like traditional data set. The cause for this is that the traditional core analysis does average the entire foot section, while the 
minipermeameter reads the permeability at each quarter foot specific point, and on the surface.

The minipermeameter measures its permeability at a specific point on the core every quarter foot. This is done by the measure, record, and move technique of the device. (Figure 15).

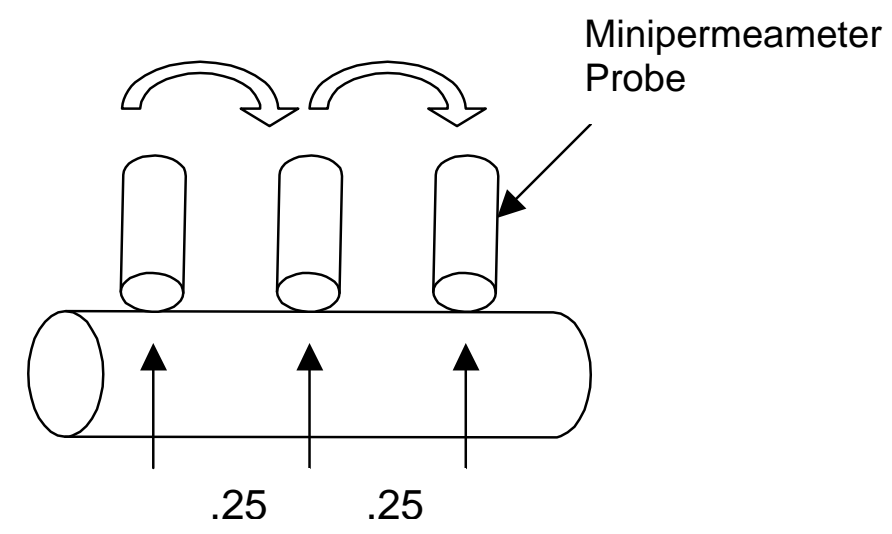

Figure 15. Illustation of measuring procedure of Minipermeameter

This technique also conflicts with the log data. The gamma ray and density logs are measured as the logging tool is pulled, at a constant speed, up the wellbore, measuring values constantly and effectively creating a moving average. The logging tool also penetrates the formation somewhat radiantly and not straightly, adding to the moving average occurrence. See Figure 16.

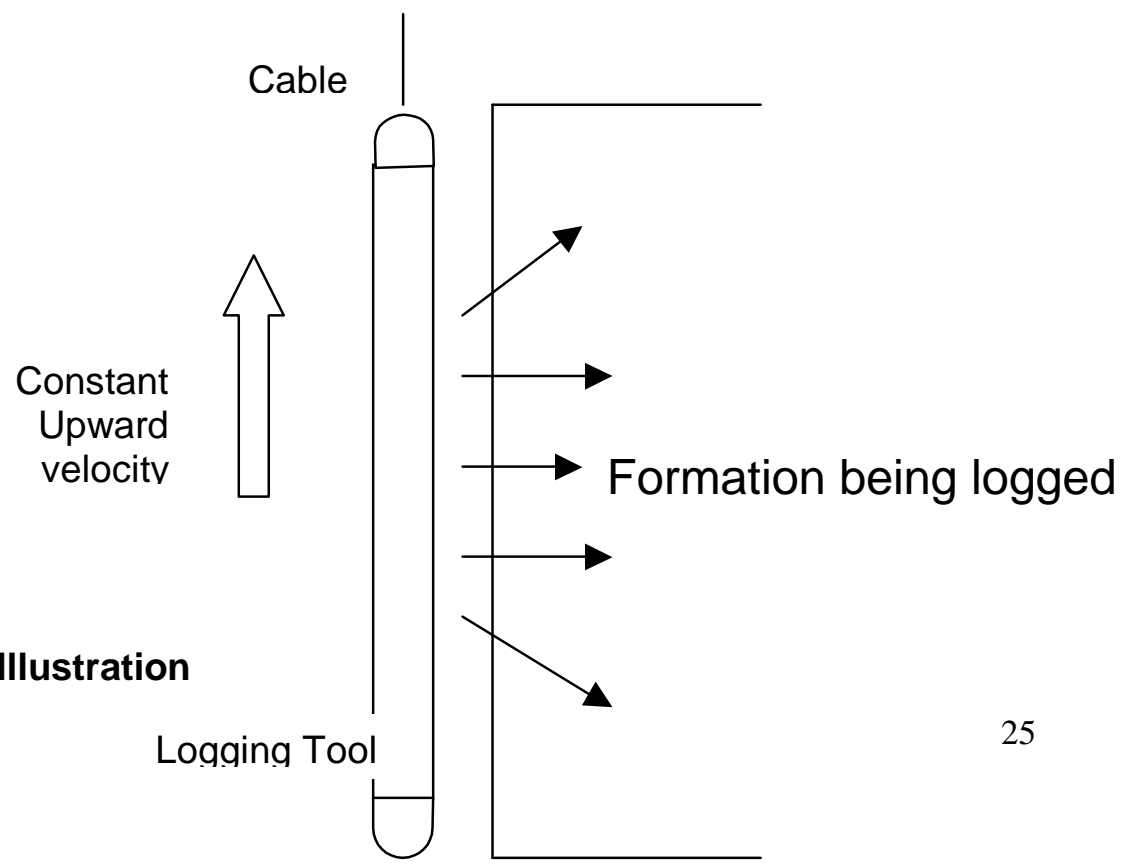


The solution to this problem was to average the minipermeameter data, on a moving basis which would be more compatible with the core and log data. The procedure for this was done by adding the mini-permeability value of concern, the value .25 feet above, and the value .25 feet below and dividing by three.

Mini-Perm Avg $_{n}=\left(\right.$ mini-perm $_{n-25}+$ mini-perm $_{n}+$ mini-perm $\left._{n+.25}\right) / 3$

This was carried out for each quarter foot value creating a moving average which was more compatible to the core and log data. Figures $17 \& 18$, show the original minipermeameter values and the moving minipermeameter average values for Cored Well Number One, demonstrating the smoothing effect of the data without affecting the general trend. The Appendix shows the actual values and graphs for each cored well.

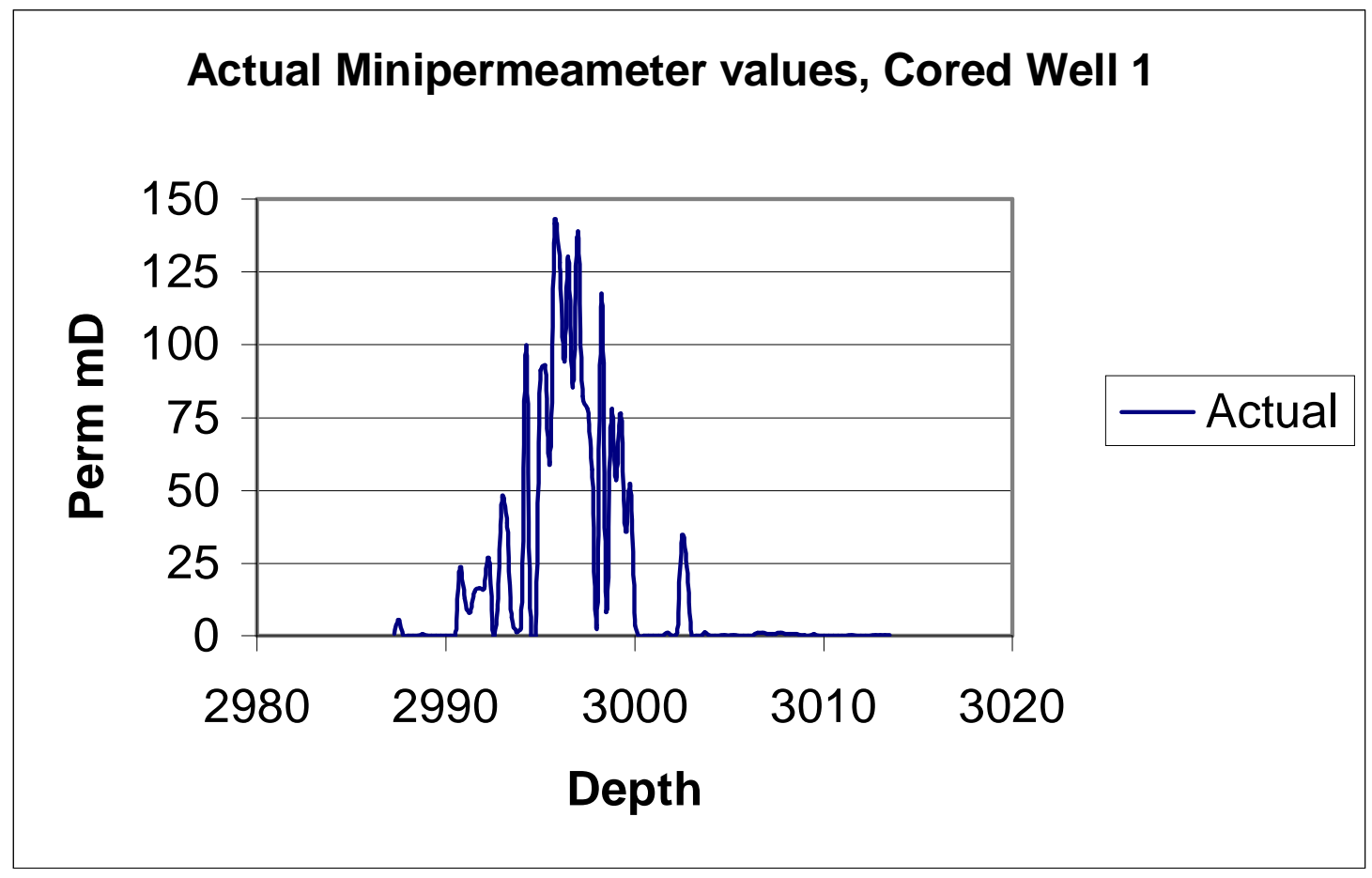

Figure 17. Actual Minipermeameter values for $\mathrm{Cw} 1$ 


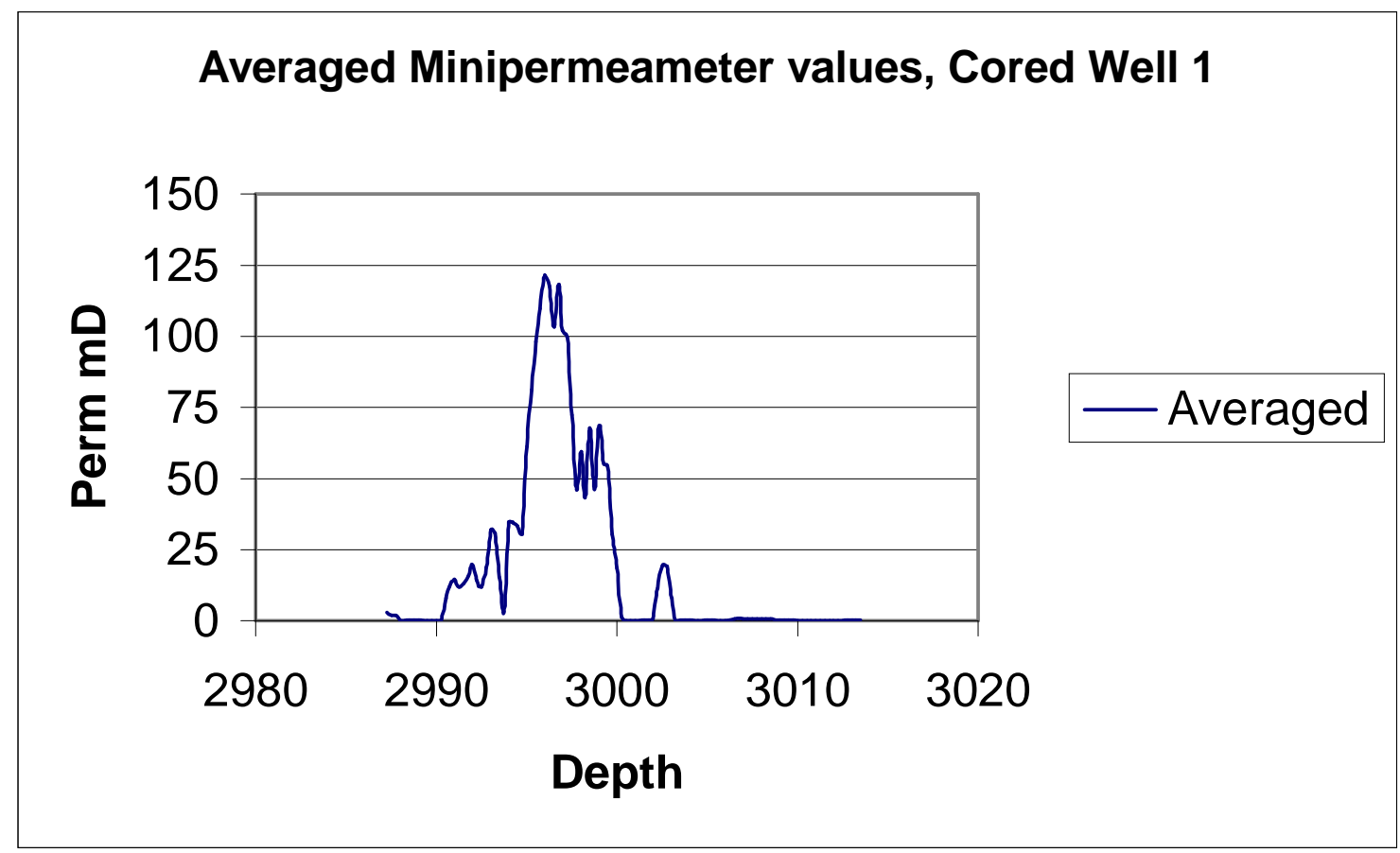

Figure 18. Average Minipermeameter Values for Cw 1

\subsection{Overview of Neural Network Developments:}

The procedure to achieve a good prediction of reservoir permeability from minipermeability data and log data can be described as three steps or processes. The first is the development an artificial neural network (Neural Network One) designed and trained to convert minipermeameter values into core permeability values This net was applied to all of the cored wells to produce a data set of core permeabilities for every quarter foot of each cored well, an improvement over the one foot resolution previously available. With this new and more useful data, finally an additional artificial neural network (Neural Network Number Two) was developed to predict reservoir 
permeabilities from log data. Figure 19 is a flowchart designed to give a visual representation of this entire process.

Neural Network One created to convert Minipermeameter values into core permeability

The application of Neural Network One to generate core permeabilities for every quarter foot of each cored well

Neural Network Two created to predict reservoir permeability from log data, with newly generated core permeabilities as the targeted output

Figure 19. Flow Chart of Neural Network Developments 


\subsection{Neural Network One Development:}

The purpose of Neural Network One (NN1) was to convert minipermeameter values into core permeability. It was determined from earlier studies that log data responds very favorably to core permeability values, but not so favorably to minipermeameter values.

The reason that the log data responds so favorably to the core permeability and not to the minipermeameter values is because of the penetration of approximately one foot into the formation by the logging tool. Core permeability is near perfect representation of the formation because the entire core plug is tested and an average permeability is obtained for the core's width, height, and diameter (the entire volume). The minipermeameter records its permeability on the surface with little penetration, which clearly diverges from the log data.

For the these reasons, phase one of the structure used to solve the overall problem of predicting reservoir permeability from minipermeameter readings was to convert the mini-permeability into core permeability. This was done by utilizing log data and known mini and core permeabilities to design an artificial neural network for the expressed purpose. The available log data consisted of Gamma Ray, Density, Gamma Ray Slope, Density Slope, GR $2^{\text {nd }}$, RHOB $2^{\text {nd }}$, GR Baseline, RHOB Baseline. These along with Depth, $\mathrm{X}$ coordinate, $\mathrm{Y}$ coordinate, and minipermeameter values comprised the net's inputs. The core permeability made up the singular actual output.

In attempting to obtain an accurate relationship between mini-permeability and core permeability, several artificial neural network architectures (structures) and paradigms were considered and tried. Using criteria equally weighting accuracy, determined by $\mathrm{R}$ squared values, and generalization, determined by consistency of those values when omitting different wells in production sets, a three-layer back 
propagation network was selected. This network consisted of three slabs in the middle layer with each slab having a different activation function. As briefly stated earlier, this architecture is very useful in forecasting and/or prediction because of its capability to generalize so well on a wide selection of problems. ${ }^{3,10}$ The reason given for this characteristic is that when each middle slab is appointed a different activation function, the network is able to uncover different and often unique features of the input vector simultaneously. Theoretically, this prevents the net from overemphasizing on an insignificant input.

The activation functions used in each middle slap consisted of two probabilistic functions and one sigmoid function, Gaussian, Gaussian complement, and tangent hyperbolic respectively. The probabilistic functions (Gaussian and Gaussian complement) show some benefits over other functions as they are not increasing. The Gaussian function tends to ignore the influential characteristics of the input extremes, very high values or very low values. This gives the network the ability to see the important characteristics within the mid-ranged input values. This is perfectly complimented, as its name implies, by the Gaussian complement function, which gives the network a good view of the meaningful characteristics at the extreme low and high ends of the input set. The sigmoid function Tangent hyperbolic adds to the overview by squeezing the input data and only producing a strong output if an input is above normal. ${ }^{10}$

The input and output layers consist of one slab each. Options are also available to set activation functions on these slabs. The input slab's input function was selected to be linear $[-1,1]$, which is standard procedure and just causes the network to pass the input data directly, unchanged, to the middle layer. The output slab was given a logistic 
output function, which helps to accent the range of the inputs when receiving information from the middle layer. Also, being a sigmoid function it adds another relationship of the function to the derivative value.

The number of neurons in each slab are the only remaining part of the structure to be considered. The input layer, which consists of only one slab, has its neurons set to correspond with the number of inputs entered into the artificial neural network. For Neural Network One this is 12. Similarly, the output layer, with one slab, has its neurons equal to the number of desired outputs. This is core permeability only and is also set to one.

The middle layer is a bit more complex in determining the number of neurons for each of the three slabs. The program used determines this number by applying the following equation.

$$
\text { number of neurons }=\frac{\text { (inputs }+ \text { outputs) }}{2}+\sqrt{\text { data points in training set }}
$$

Applying this equation to the data used in Neural Network One this number was determined to be approximately 16 and so the entire middle layer consists of 15 neurons, five in each slab in order to give each one equal weight in its contribution to the network. In conclusion, one neuron was used for the input slab, five for each middle slab and finally one for the output slab. The entire architecture, structure, activation functions and neurons are shown in Figure 20. 


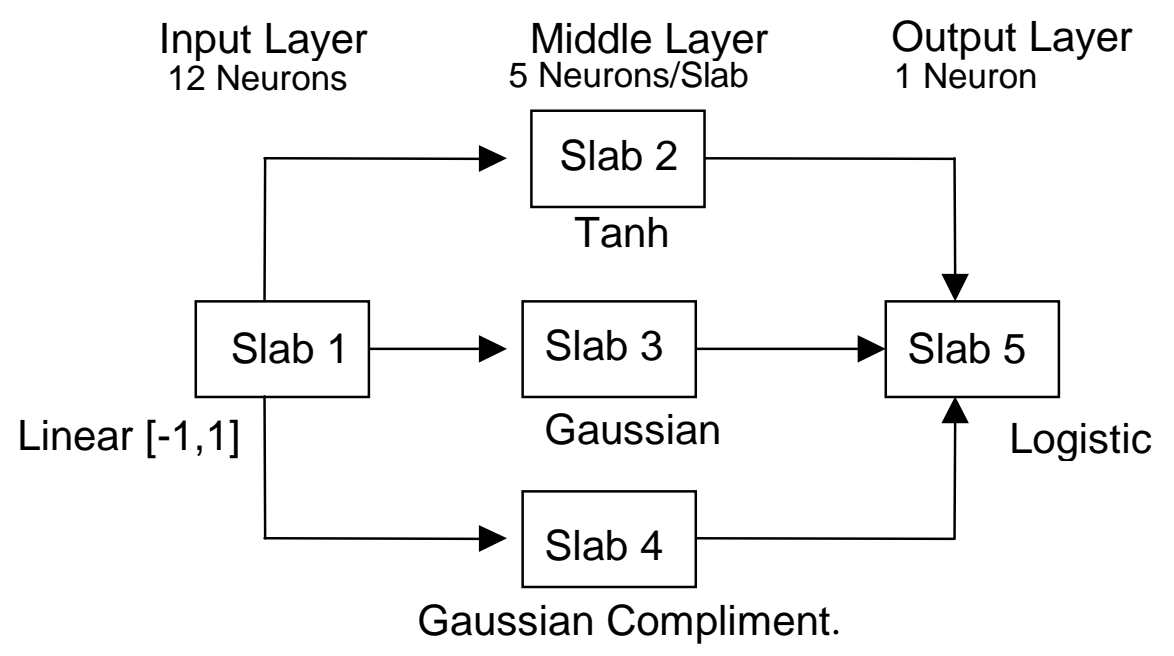

Figure 20. Preliminary Structure of Neural Network One

Many factors can affect the training of an artificial neural network and this section will present procedures and limits used in Neural Network One. It is also at this point when certain inputs will be organized and possibility eliminated based on their contribution values. The training of this artificial neural network took place within an initial total of 93 training points for all five cored wells.

A test set for the network to use in a comparison capacity had to be extracted. There are several methods available to produce this test set. It can be manually selected, be extracted as all the data points for a specific well, or randomly chosen. For the purposes of this study the random selection method appeared to be the appropriate solution. The test set was chosen as 20 percent of all the data points, producing a group 
of 18 test points representing all wells which were inputted into the network. This left 75 points in the training set. Each time the Neural Network One runs through an entire calibration interval the results are compared to this test set in order to produce an internal average error. The network constantly attempts to reduce this error. The problem which this network attempts to solve is considered very complex and noisy, for this reason the learning rate and momentum were both set at .1 , to allow for the observance of more subtlety important features, and prevent the problem of overlearning.

The values used to initialize the weight of all connection links were set at the default of 0.3 . The calibration interval is the number of patterns processed by the artificial neural network before it is compared to the test set. This value is also variable and can be decided by the designer of the network. The default is set to 200 , but it was discovered by experience that a process within the neural network construction program known as TurboProp produced better learning results. TurboProp automatically sets the calibration interval equal to the number of training patterns. In other words, this method causes the network to check the test set once each time it goes through all of the training patterns.

The learning of the artificial neural network also has to have a stop criterion to prevent over learning or memorization of the presented outputs. This was selected to be as follows: When the number of training events reaches 20,000 without a new minimum internal error on the test set, the training was terminated. It was also decided that the network would be automatically saved on the minimum internal error of the test set. 
Through several trials, the $\mathrm{R}$ squared values (test and training sets), and contribution values, defined as the representation of an input's strengths in predicting the output, were analyzed. This analysis suggested that three inputs were not useful to the prediction capabilities of the network, and may actually be degrading those capabilities somewhat. These inputs were Gamma Ray Second Derivative, Density Second Derivative, and Density Baseline. With this knowledge, they were omitted. With this change in inputs, the number of neurons in the input slab was changed to nine. Also, through trial and error it was discovered that a configuration with six neurons in each middle slab produced better results. This left the final structure as shown in Figure 21.

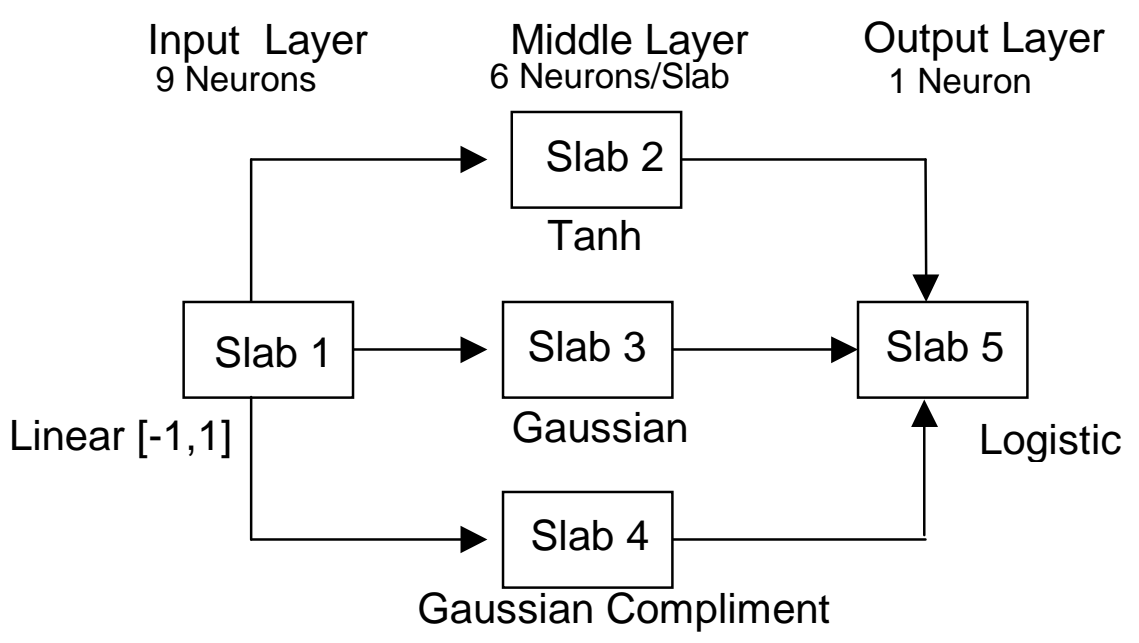

Figure 21. Final Structure of NN One 


\subsection{Application of Neural Network One:}

The second phase of the process was to apply the newly developed Neural Network One to calculate core permeability on the five cored wells for each quarter foot interval. See Figure 19. Average minipermeameter values and log data were used as inputs. This was done by creating a DLL file which takes the presented data (log and minipermeameter values) from the cored wells and applies Neural Network One. The CALL function in Microsoft Excel was used to access this DLL file and entered into each interval's output row on the spreadsheet. See Figure 22.

\begin{tabular}{|c|c|c|c|c|c|c|c|c|c|} 
Density & $\begin{array}{c}\text { Gamma } \\
\text { ray }\end{array}$ & $\begin{array}{c}\text { Density } \\
\text { slope }\end{array}$ & $\begin{array}{c}\text { Gamma ray } \\
\text { slope }\end{array}$ & $\begin{array}{c}\text { Gamma ray } \\
\text { baseline }\end{array}$ & $\mathrm{x}$ coord & y coord & $\begin{array}{c}\text { Avg } \\
\text { Miniperm }\end{array}$ & Depth & $\begin{array}{c}\text { core } \\
\text { perm }\end{array}$ \\
\hline input & input & input & input & input & input & input & input & input & CALL NN1.DLL \\
& & & & & & & & & \\
\end{tabular}

Figure 22. Description of Excel Spreadsheet used to generate core permeabilities from log data and Minipermeameter values

The generated core permeability values are shown for all cored wells in Appendix . These values would now be used in the construction of Neural Network Two. 


\subsection{Neural Network Two Development:}

The purpose of Neural Network 2 (NN2) is to predict reservoir permeability from log data, using the newly generated core permeabilities as a targeted output. In this section the construction of Neural Network Two is discussed. Several of the earlier points used in the Neural Network One construction are again presented. They continue to hold true for this new network, and must be reiterated in order to understand why certain choices were made.

The available log data again consisted of Gamma Ray, Density, Gamma Ray Slope, Density Slope, GR $2^{\text {nd }}$ Slope, RHOB $2^{\text {nd }}$ Slope, GR Baseline, RHOB Baseline. These along with Depth, $\mathrm{X}$ coordinates, and $\mathrm{Y}$ coordinates, comprised the net's inputs. The core permeability generated from the application of Neural Network One (at quarter foot intervals) made up the singular actual output.

In attempting to obtain an accurate relationship between log data and the new generated core permeability, several artificial neural network architectures (structures) and paradigms were considered and tried. Using criteria equally weighting accuracy, determined by $\mathrm{R}$ squared values, and generalization, determined by the consistency of those values when omitting different wells in production sets, a three-layer back propagation network was selected. The chosen back propagation network has three slabs in the middle layer, each having a different activation function. As stated several times earlier, this architecture is useful in forecasting and/or prediction., ${ }^{3,10}$

The activation functions chosen for the middle slaps consisted of two probabilistic functions, Gaussian and Gaussian complement, and one sigmoid function, tangent hyperbolic. The benefits of these activation functions were previously discussed in the development of Neural Network One. 
The input and output layers consist of one slab each. A linear $[-1,1]$ function was used in the input layer, this is standard procedure and causes the network to pass the input data directly and unchanged, to the middle layer. A logistic function was used in the output slab.

The number of neurons in each slab are again the only remaining part of the structure to be considered. The input layer, which consists of only one slab, has its neurons set to correspond with the number of inputs entered into the artificial neural network. For Neural Network Two this is 11. Similarly, the output layer, with one slab, has its neurons equal to the number of desired outputs. This is core permeability only and is also set to one.

The program used determines the number of neurons for each of the three middle slaps by applying the following equation.

$$
\text { number of neurons }=\frac{\text { (inputs }+ \text { outputs) }}{2}+\sqrt{\text { data points in training set }}
$$

Applying this equation to the data used in Neural Network Two and the and this number was determined to be approximately 24 and so the entire middle layer consists of 24 neurons eight in each slab in order to give each one equal weight in its contribution to the network. In conclusion, one neuron was used for the input slab, eight for each middle slab and finally one for the output slab. The entire preliminary architecture, structure, activation functions, and number of neurons are shown in Figure 23. 


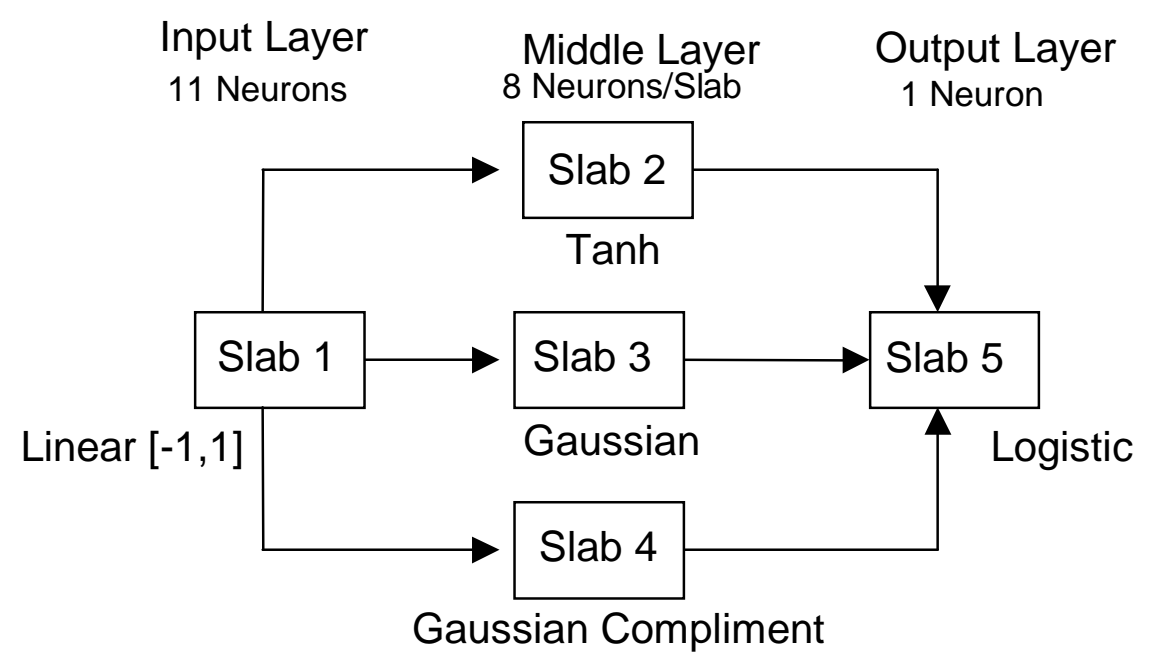

Figure 23. Preliminary Structure of NN Two

Many factors can affect the training of an artificial neural network and this section will present procedures and limits used in the Neural Network Two. Is also at this point when certain inputs can be organized and possibility eliminated based on their contribution values. The training of this artificial neural network took place with an initial total of 396 training points for all five cored wells.

A test set for the network to use in a comparison capacity was extracted by a random selection method. The test set was chosen as 20 percent of all the data points, producing a group of 79 test points representing all wells which were inputted into the network. This left 317 points in the training set. Each time the Neural Network Two runs through an entire calibration interval the results are compared to this test set in order to produce an internal average error. The network constantly attempts to reduce this error. The problem which this network attempts to solve is again considered very complex and noisy, for this reason the learning rate and momentum were both set at 0.1 , to allow for 
the observance of more subtlety important features, and to prevent the problem of overlearning.

The values used to initialize the weight of all connection links were set at the default of 0.3 . The calibration interval is the number of patterns processed by the artificial neural network before it is compared to the test set. This value is also variable and can be decided by the designer of the network. The default is set to 200 , but it was discovered by experience that a process within the neural network construction program known as TurboProp produced better learning results. TurboProp automatically sets the calibration interval equal to the number of training patterns. In other words, this method causes the network to check the test set once each time it goes through all of the training patterns.

The learning of the artificial neural network also has to have a stop criterion. This was selected to be as follows: When the number of training events reaches 20,000 without a new minimum internal error on the test set, the training was terminated. It was also decided that the network would be automatically saved on the minimum internal error of the test set.

Through several trials the $\mathrm{R}$ squared values (test and training sets), and contribution values, defined as the representation of an input's strength in predicting the output, were analyzed. This analysis suggested that some inputs were not useful to the prediction capabilities of the network. These inputs, the same as in NN One, were Gamma Ray Second Derivative, Density Second Derivative, and Density Baseline. These inputs were again omitted. With this change in inputs, the number of neurons in the input slab was changed to eight. This change in the number of inputs reduced the 
default middle layer neurons to 22 allocating each slab with seven neurons. Again, through trial and error several other middle layer neuron configurations were attempted, but unlike the Neural Network One it was concluded that the default number of 7 neurons for each slab produced the best results. This left the final structure as shown in Figure 24.

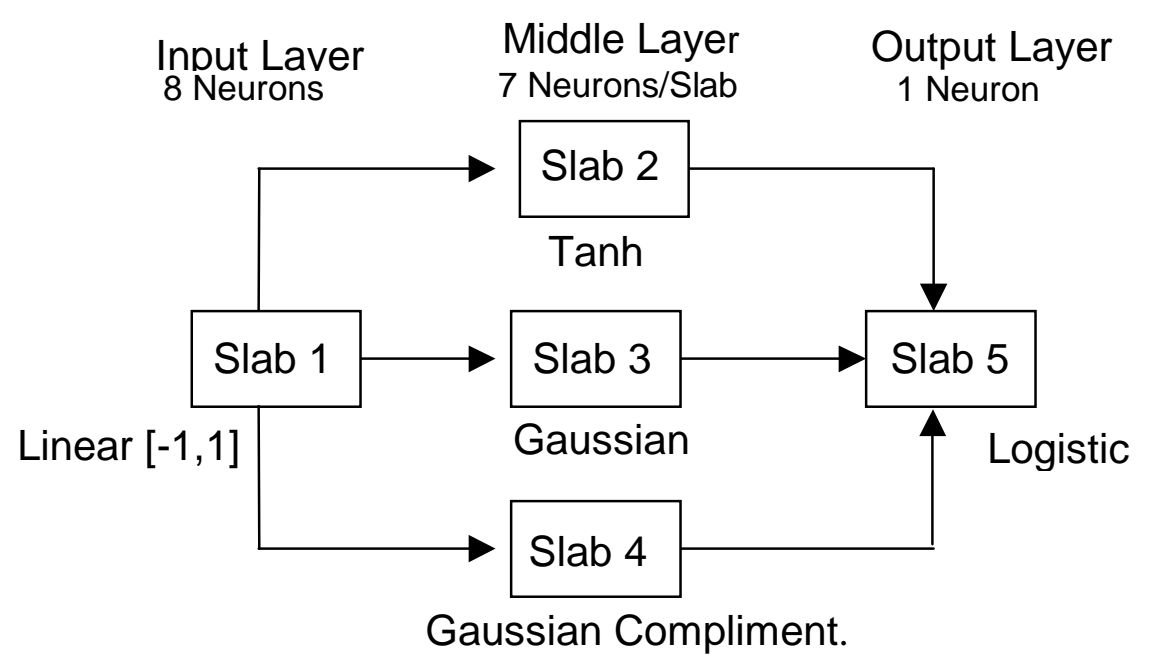

Figure 24. Final Structure of NN Two 


\section{Chapter Four: Results and Discussion}

\subsection{Results of Neural Network One:}

Applying the final structure of Neural Network One , produced the following results:

\section{For the Pattern Set:}

Patterns processed: 93

Output:

R squared:.......................9.9793

r squared:...................... 0.9795

Mean squared error:............94.328

Mean absolute error:...........5.233

Min. absolute error:............ 0

Max. absolute error:...........52.811

Correlation coefficient $r$....... 0.9897 


\section{For the Test Set:}

Patterns processed: 18

Output:

R squared:

.0 .9893

r squared: 0.9896

Mean squared error:. 75.174

Mean absolute error: .4 .826

Min. absolute error: .0 .026

Max. absolute error: .26 .867

Correlation coefficient $r: \ldots \ldots . . .0 .9948$

It is important for one to pay close attention to the $\mathrm{R}$ squared value of the pattern set. With 1.0 being a perfect match, this neural network produced an $\mathrm{R}$ squared of .9793. The training and test set values are also good with the training equal to .9741 and the test equal to .9893 . In order to illustrate this correlation several graphs are presented. The first is a plot of neural network output and actual data vs. depth for Cored Well Number One. 


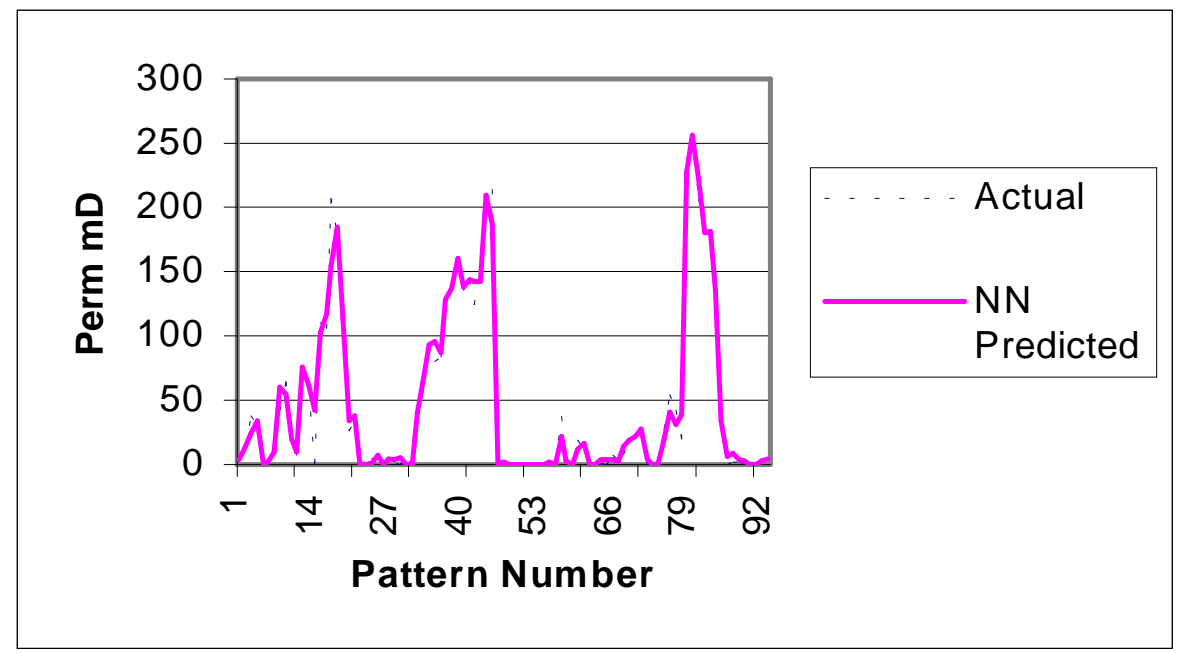

Figure 25. Actual \& NN Predicted Perm. for NN One Applied to Pattern Set

Also, to show the correlation the next three graphs plot actuals vs. neural network outputs for each set, pattern, training, and test.

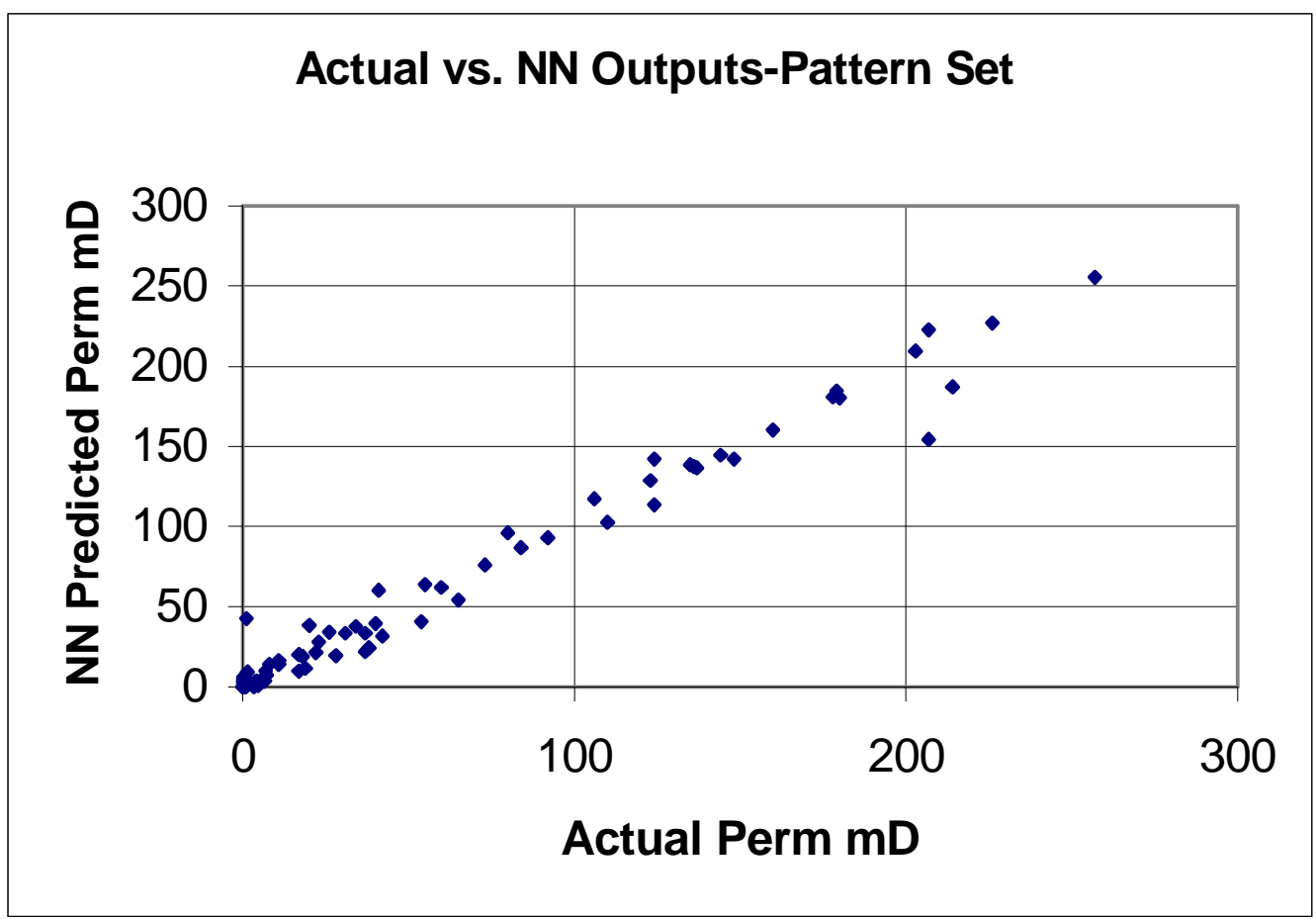

Figure 26. Actual vs. NN Predicted for NN One Applied to Pattern Set 


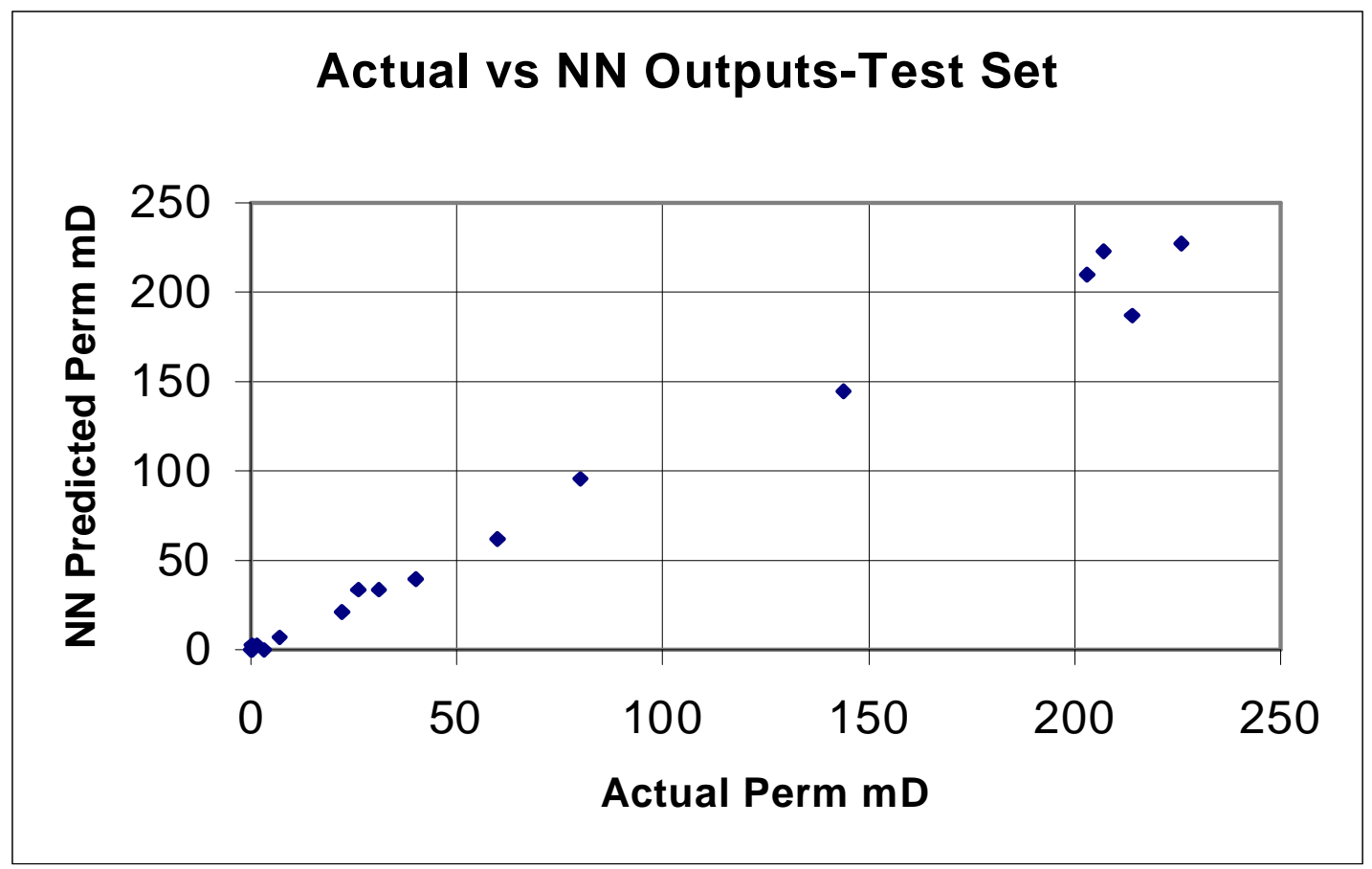

Figure 27. Actual vs. NN Predicted for NN One Applied to Test Set

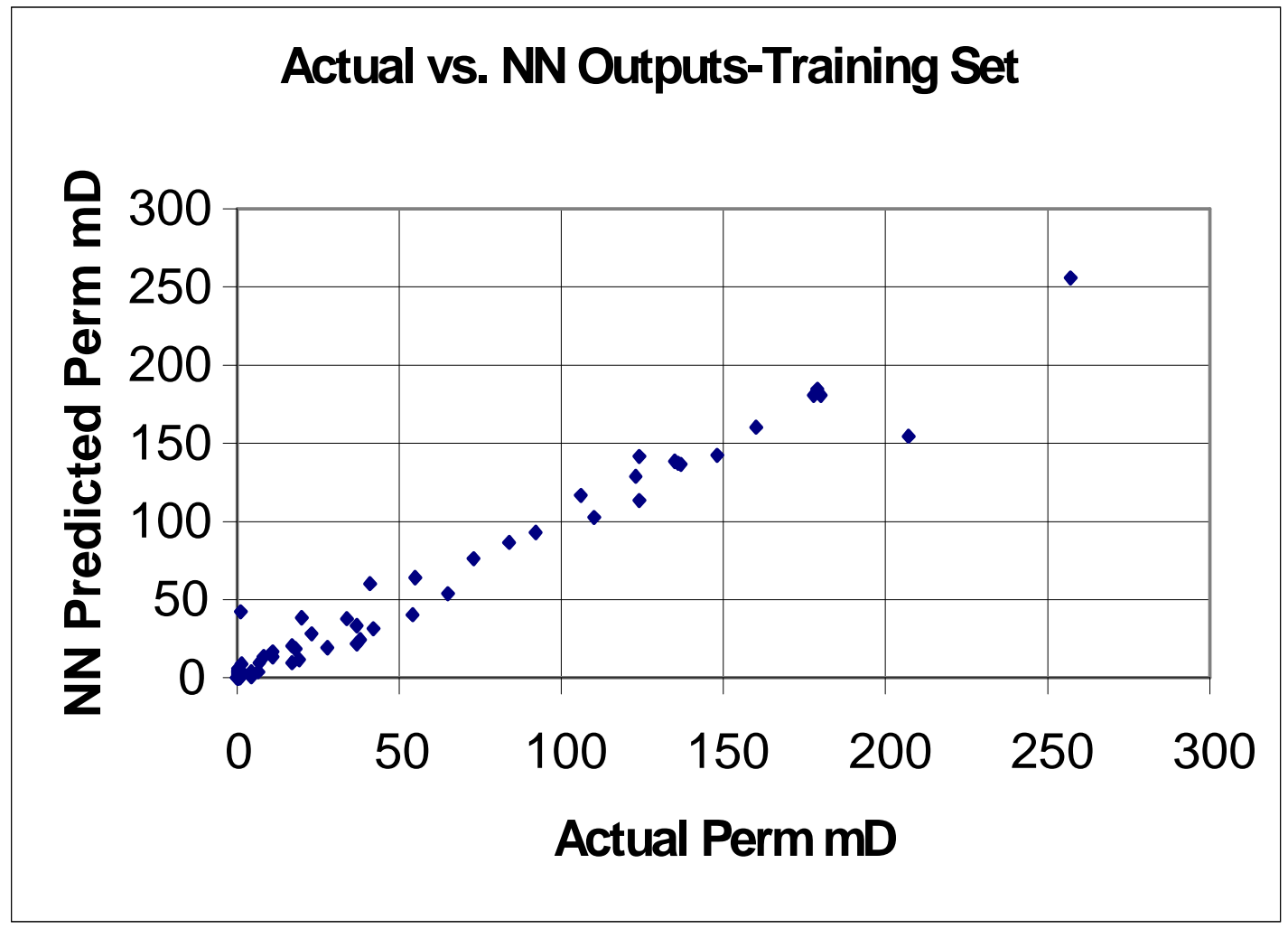

Figure 28. Actual vs. NN Predicted for NN One Applied to Training Set 
The contribution values for each input are shown in Table 1 and have been calculated to a percentile scale with each value based on the total of 100 percent. As it is observed, Density and the $X$ coordinate produce the strongest inputs $(18.23 \%$ and $17.36 \%$ respectively) while the $Y$ coordinate and Depth represent the weakest $(6.11 \%$ and $4.43 \%$ ), although they were determined to be valuable enough not to omit.

Table 1. Contribution values of inputs in NN One

\begin{tabular}{|c|c|c|}
\hline Input Name & Contribution Value & Percent of $100 \%$ Contribution \\
\hline density & 0.182267353 & 18.22673529 \\
\hline x Coord & 0.173610017 & 17.36100167 \\
\hline D Slope & 0.153621957 & 15.36219567 \\
\hline GR Slope & 0.140633255 & 14.06332552 \\
\hline Avg Perm & 0.085468158 & 8.546815813 \\
\hline GR BL & 0.083171234 & 8.317123353 \\
\hline GR & 0.075840317 & 7.584031671 \\
\hline y Coord & 0.061051726 & 6.105172634 \\
\hline DEPTH & 0.044336036 & 4.433603585 \\
\hline
\end{tabular}




\subsection{Neural Network One Additional Verification (Production Sets):}

The use of back-propagation models have historically been known for their ability to predict and generalize well, partly because the use of test sets ensures this. Also, the $\mathrm{R}$ squared value is a great indicator of prediction ability. These points alone, although representative, are not enough to verify a network.

It is central to the credibility of the designed artificial neural network to verify that the results can be reproduced consistently. This is generally done through the use of production sets. These production sets are data within the entire pattern set which are excluded from the training process. The network never sees them and thus, if predictions remain acceptable, adds to its desired credibility.

To achieve this verification several additional artificial neural networks were developed based on the same structure as the Neural Network Two. This means that generally the same type, structure, activation functions, initial weights, neurons, learning rate, momentum, calibration interval, and stop criterion were used in these new networks. The differences are in the exclusion of the production sets from the pattern sets. Again there are several ways, as with the test sets, to achieve extraction. The production sets may be selected randomly, manually chosen, or taken as an entire well. The method of leaving out an entire well was chosen. An important characteristic to consider is that with so few cored wells available, leaving out one well will undoubtedly reduce some of the features essential in the Neural Network One's ability to perform. Keeping this in mind, production sets with the lowest $R$ squared values imply wells that may provide very meaningful characteristics to the main artificial neural network. The construction of these verification nets produced the following results in table 2 . 
Table 2. R Squared Values for the Verification Networks of NN One

\begin{tabular}{|c|c|c|c|}
\hline $\begin{array}{c}\text { Well in Production } \\
\text { Set }\end{array}$ & $\begin{array}{c}\text { R Squared } \\
\text { Pattern Set }\end{array}$ & $\begin{array}{c}\text { R Squared Test } \\
\text { Set }\end{array}$ & $\begin{array}{c}\text { R Squared } \\
\text { Production Set }\end{array}$ \\
\hline Cored Well One & .934 & .9456 & .9206 \\
\hline Cored Well Two & .9478 & .9357 & .8184 \\
\hline Cored Well Three & .9726 & .9639 & .8426 \\
\hline Cored Well Four & .9139 & .9408 & .7699 \\
\hline Cored Well Five & .9559 & .9553 & .7810 \\
\hline
\end{tabular}

The previous data indicates that the network works acceptably leaving out one cored well. Cored Well Number Four demonstrates the lowest production set well at .7699, possibility indicating its significant contribution to the main network. As an overview, these lower values can be explained by the fact that the artificial neural network trains on fewer points than the whole pattern set causing it to predict values based on less knowledge. Figures 29,30 and 31 show the graphical results of Cored Well Number One used as a production set. 


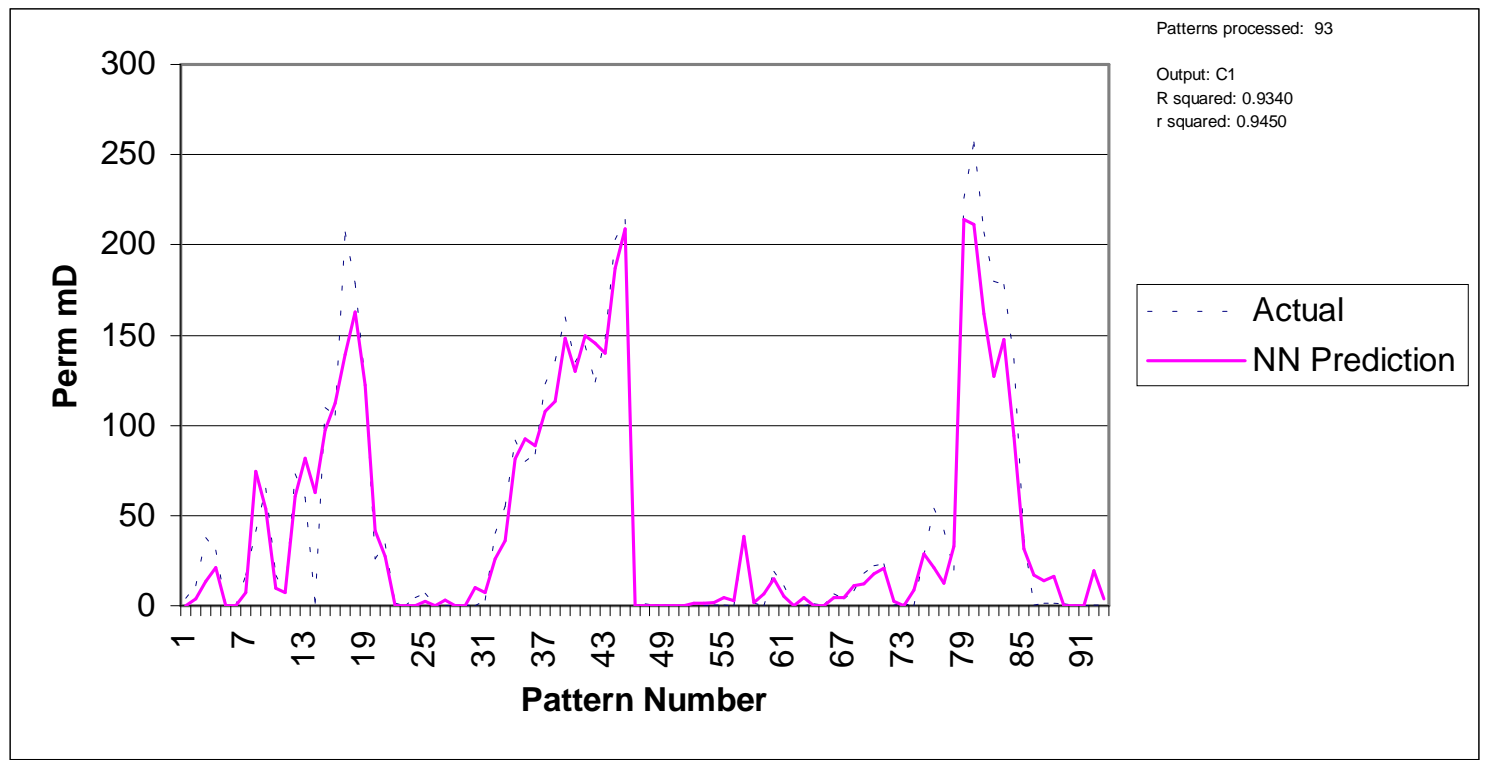

Figure 29. Cw1 used as a Production Set, Pattern Set

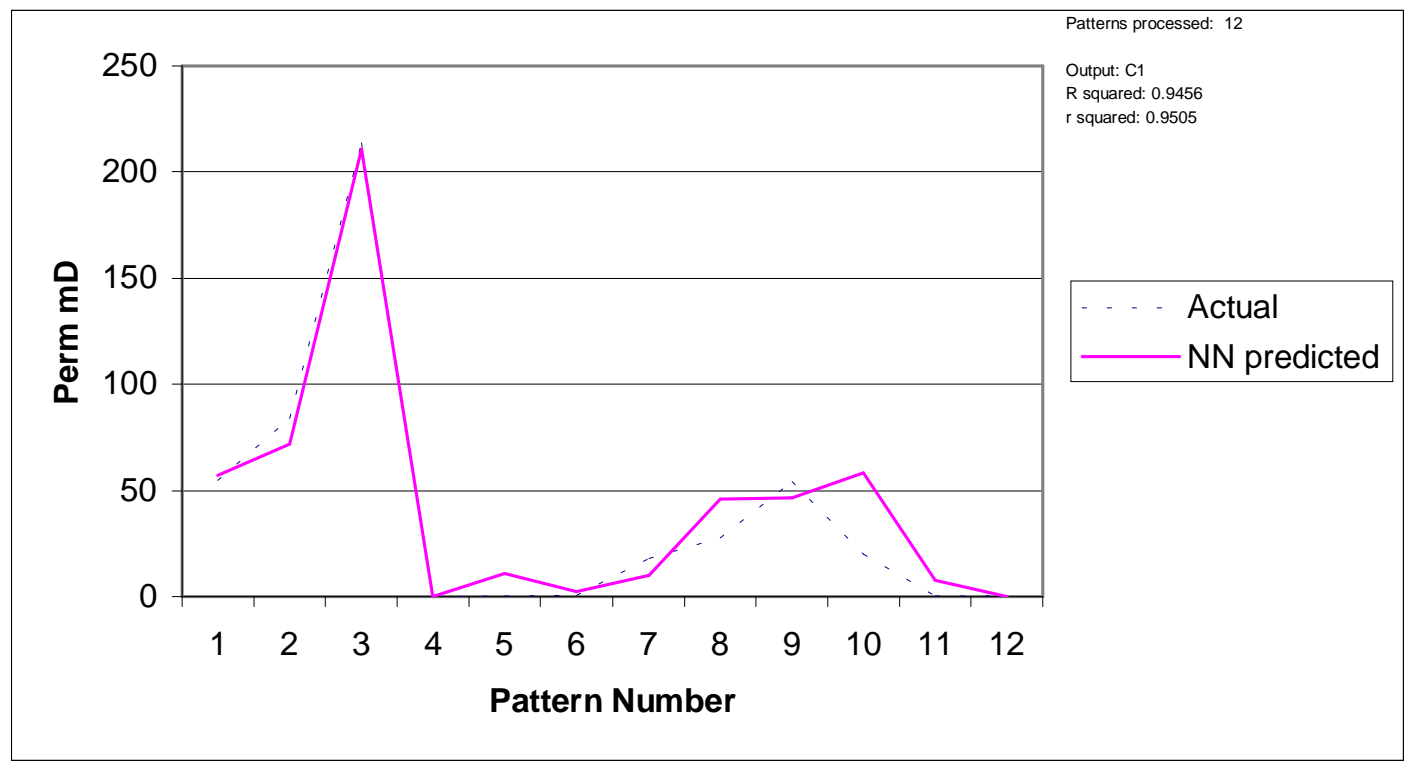

Figure 30. Cw1 used as a Production Set, Test Set 


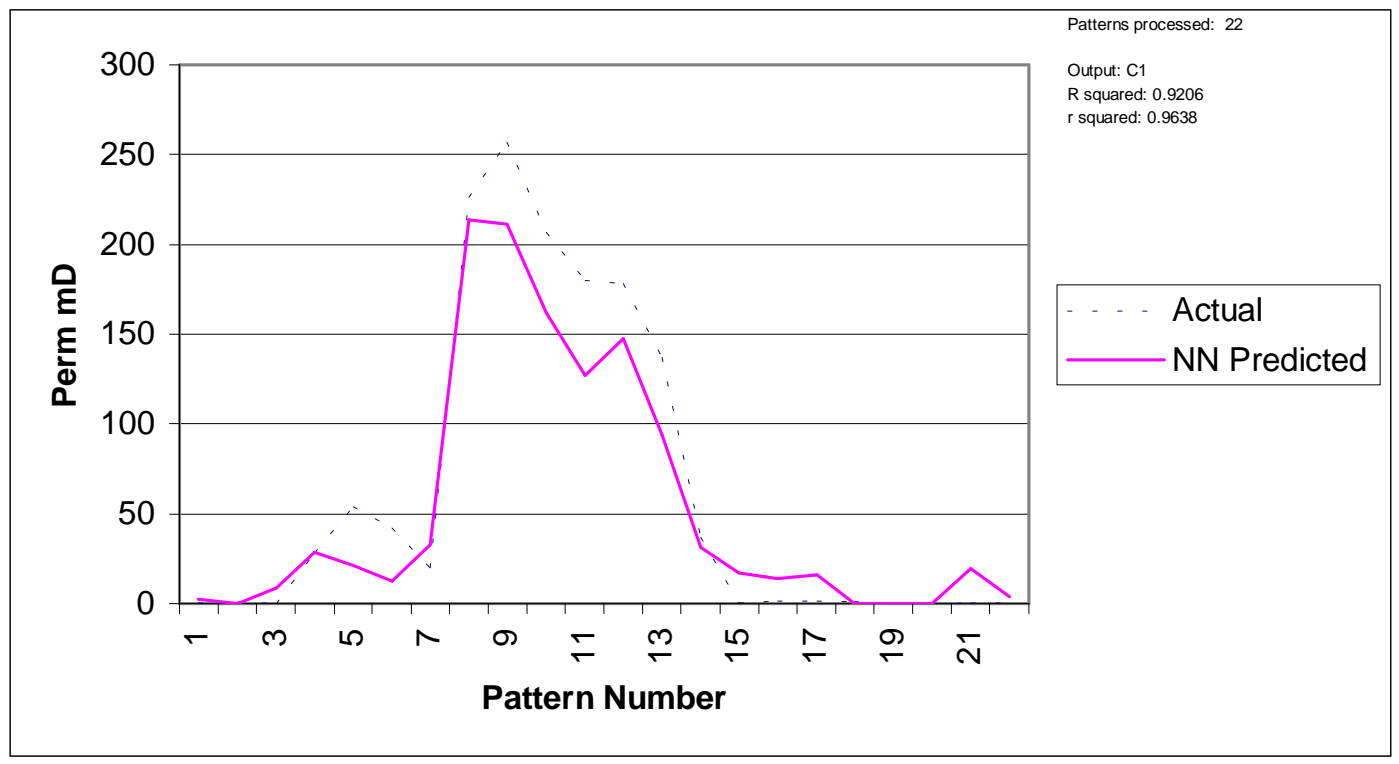

Figure 31. Cw1 used as a Production Set, Production Set

\subsection{Results of Neural Network Two:}

Applying the final structure of Neural Network Two, produced the following results:

\section{For the Pattern Set:}

Patterns processed: 396

R squared:

r squared: 0.9788

Mean squared error: .75 .002

Mean absolute error: .5 .656

Min. absolute error: 0

Max. absolute error: .42 .292

Correlation coefficient $r$........ 0.9893 


\section{For the Test Set:}

Patterns processed:

R squared:

r squared:

Mean squared error:

Mean absolute error: .5 .623

Min. absolute error: .0

Max. absolute error: .42 .292

Correlation coefficient $r$ : 0.9821

It is important for one to pay close attention to the $\mathrm{R}$ squared value of the pattern set. With 1.0 being a perfect match, this neural network produced an $\mathrm{R}$ squared of .9786. The training and test set values are also good with the training equal to .9809 and the test equal to .9645 . In order to illustrate this correlation several graphs are presented. The first is a plot of neural network output and actual data vs. depth for Cored Well Number One.

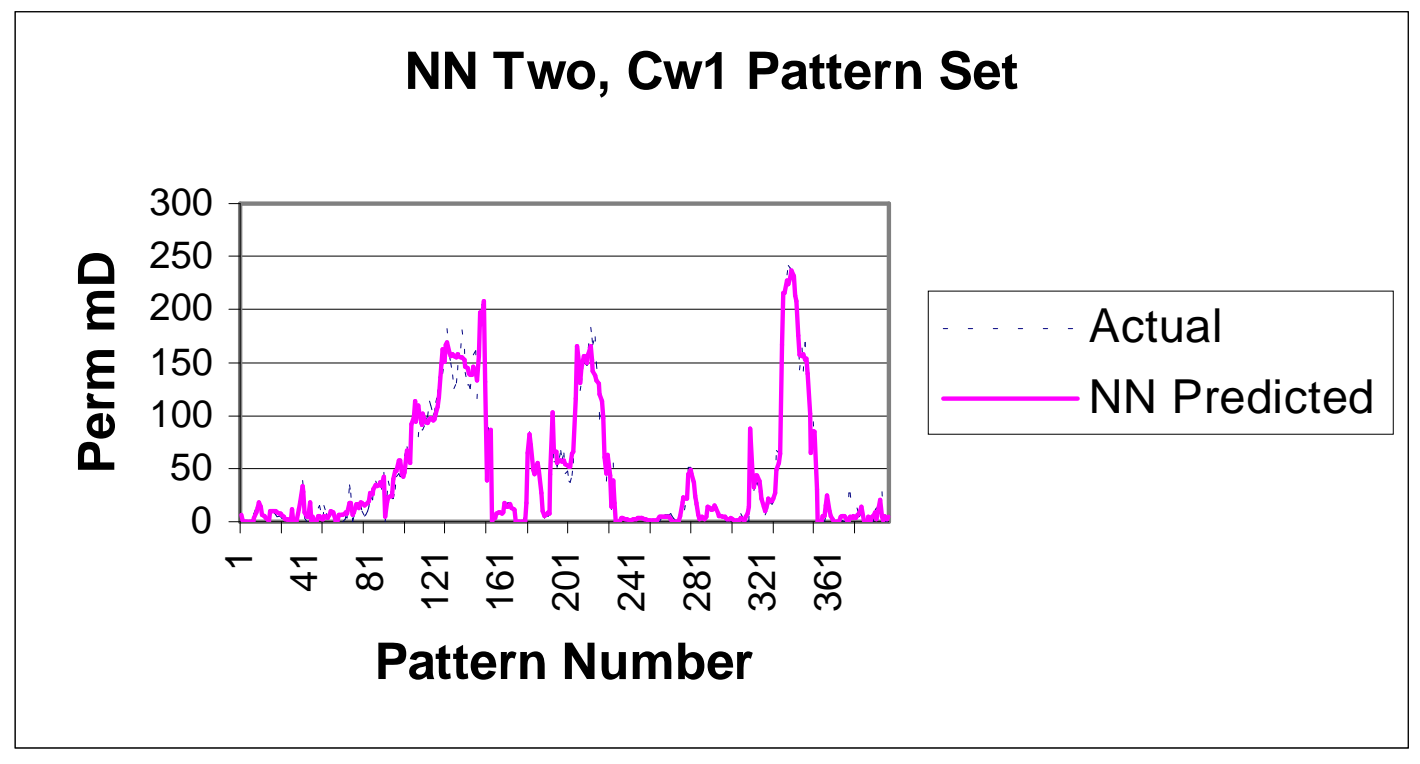

Figure 32. Actual \& NN Predicted Perm for NN Two Applied to Cw 1 Pattern Set 
Also, to show the correlation the next three graphs plot actuals vs. neural network outputs for each set, pattern, training, and test.

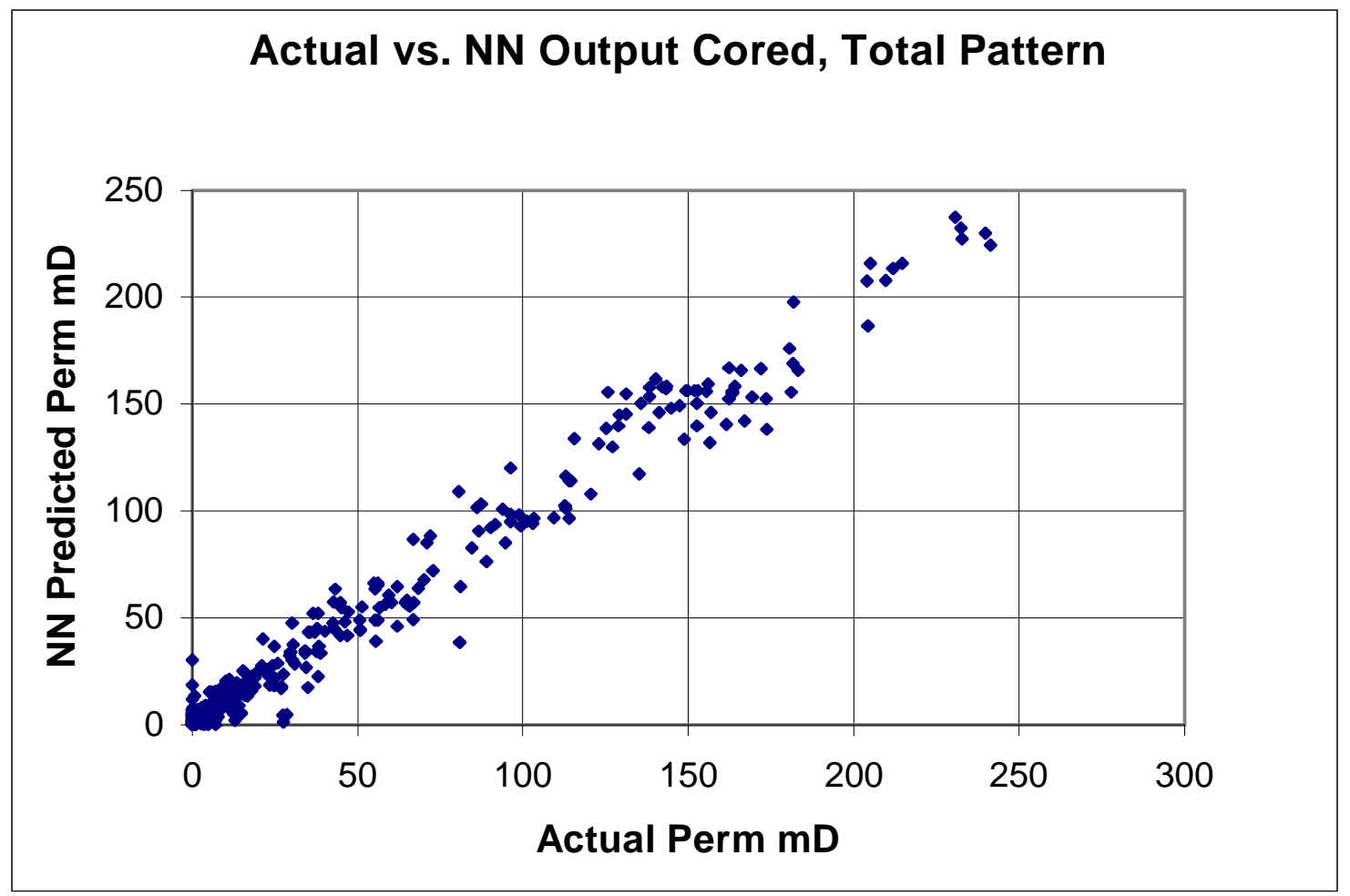

Figure 33. Actual vs. NN Predicted Perm. for NN Two Applied to Cw 1 Pattern Set

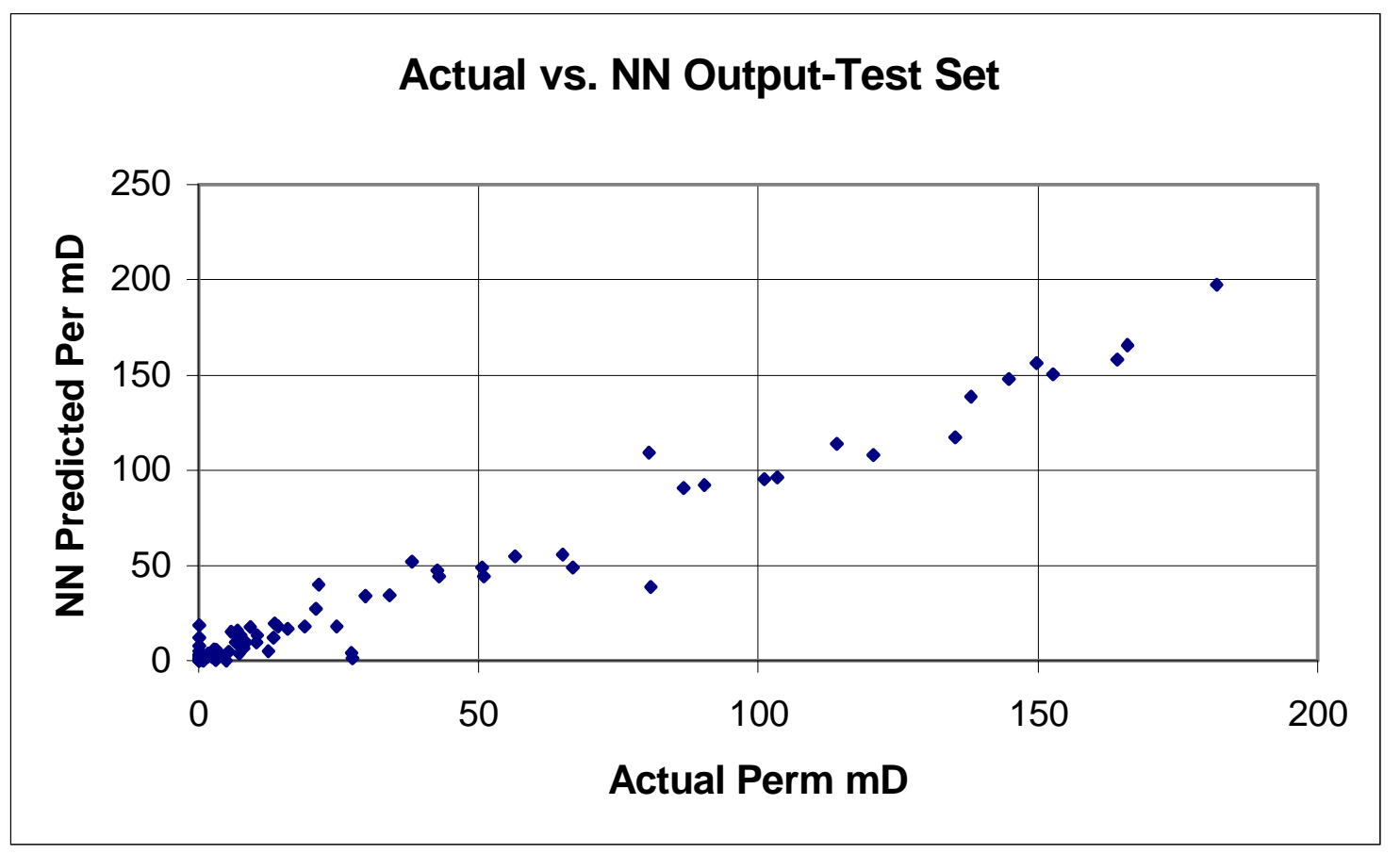

Figure 34. Actual vs. NN Predicted Perm. for NN Two Applied to Cw 1 Test Set 


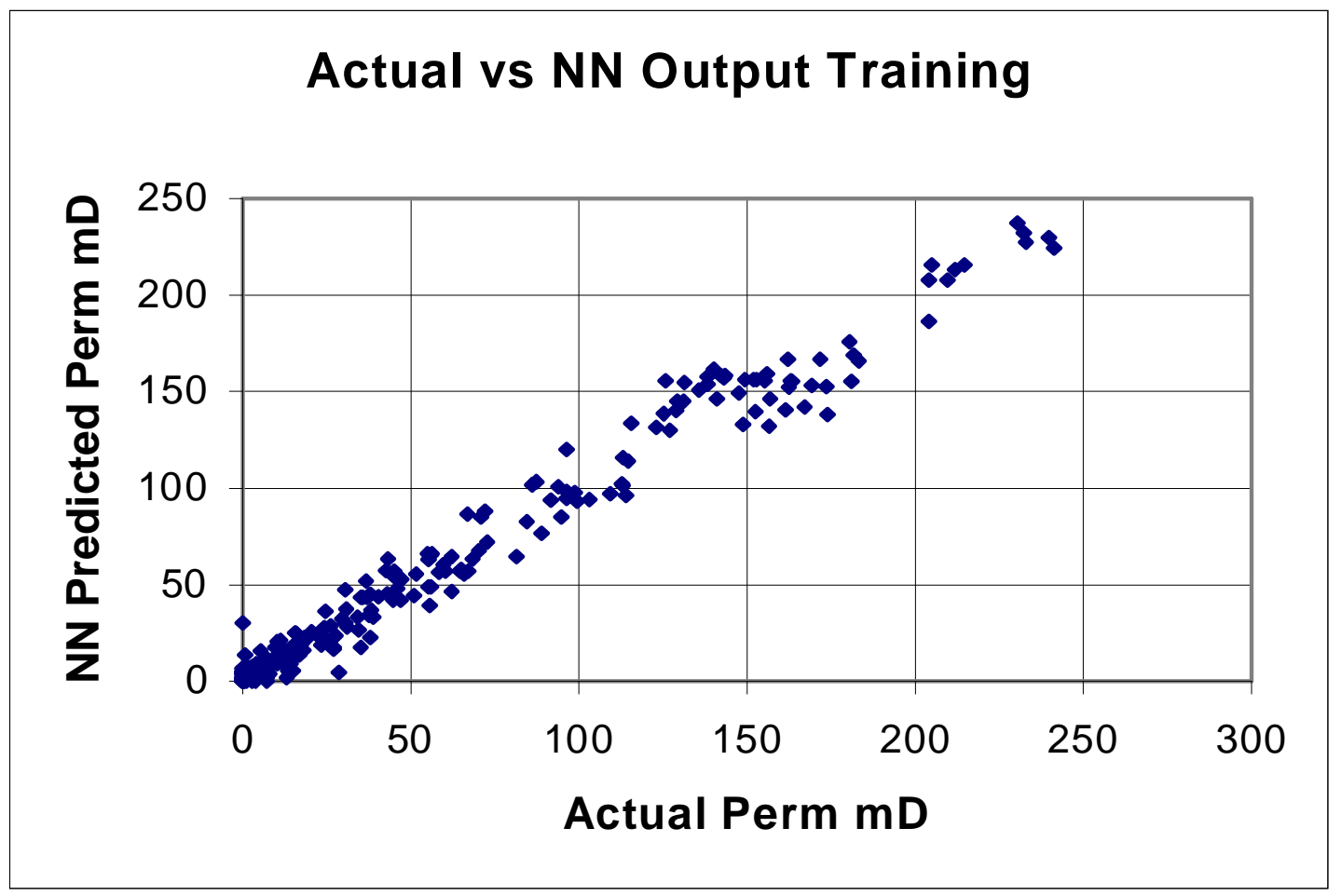

Figure 35. Actual vs. NN Predicted Perm. for NN Two Applied to Cw 1 Training Set

The contribution values for each input are shown in Table 3 and have been calculated to a percentile scale with each value based on the total of 100 percent. As it is observed, X Coordinate and Density produce the strongest inputs, (with $25.03 \%$ and $21.14 \%$ respectively) while Gamma Ray Baseline and Depth represent the weakest $(6.06 \%$ and $5.94 \%)$, although they were determined to be valuable enough not to omit. 


\section{Table 3. Contribution Values of Inputs in NN Two}

Input Name

$x$ coord

y coord

depth

GR

density

GR Slope

D slope

GR BL
Actual Contributions

0.250333309

0.073682092

0.059439644

0.091836065

0.211362079

0.125655547

0.127142802

0.060548611
Contributions out on $100 \%$

25.03333092

7.368209213

5.943964422

9.183606505

21.13620788

12.56555468

12.71428019

6.054861099

\subsection{Neural Network Two Additional Verification:}

The use of back-propagation models have historically been known for their ability to predict and generalize well, partly because the use of test sets ensures this. Also, the $\mathrm{R}$ squared value is a great indicator of prediction ability. These points alone, although representative, are not enough to verify a network. 
It is central to the credibility of the designed artificial neural network to verify that the results can be reproduced consistently. This is generally done through the use of production sets. These production sets are data within the entire pattern set which are excluded from the training process. The network never sees them and thus, if predictions remain acceptable, adds to its desired credibility.

To achieve this verification several additional artificial neural networks were developed based on the same structure as the Neural Network Two. This means that generally the same type, structure, activation functions, initial weights, neurons, learning rate, momentum, calibration interval, and stop criterion were used in these new networks. The differences are in the exclusion of the production sets from the pattern sets. Again there are several ways, as with the test sets, to achieve extraction. The production sets may be selected randomly, manually chosen, or taken as an entire well. The method of leaving out an entire well was chosen. An important characteristic to consider is that with so few cored wells available, leaving out one well will undoubtedly reduce some of the features essential in the Neural Network Two's ability to perform. Keeping this in mind, production sets with the lowest $R$ squared values may imply wells that provide very meaningful characteristics to the main artificial neural network. The construction of these verification nets produced the following results in Table 4. 
Table 4. R Squared Values for Production Networks in the Verification of NN Two

\begin{tabular}{|c|c|c|c|}
\hline $\begin{array}{c}\text { Well in Production } \\
\text { Set }\end{array}$ & $\begin{array}{c}\text { R Squared } \\
\text { Pattern Set }\end{array}$ & $\begin{array}{c}\text { R Squared Test } \\
\text { Set }\end{array}$ & $\begin{array}{c}\text { R Squared } \\
\text { Production Set }\end{array}$ \\
\hline Cored Well One & .9474 & .9511 & .9062 \\
\hline Cored Well Two & .9374 & .9341 & .8669 \\
\hline Cored Well Three & .8956 & .8915 & .7017 \\
\hline Cored Well Four & .9612 & .9556 & .8837 \\
\hline Cored Well Five & .9507 & .9133 & .5696 \\
\hline
\end{tabular}

The previous data indicates that the network works reasonably well when leaving out one cored well. Cored Well Number Five demonstrates the lowest production set well at .5696, indicating its significant contribution to the main network Neural Network Two. As an overview, these lower values can be explained by the fact that the artificial neural network trains on fewer points than in the whole pattern set causing it to predict values based on less knowledge. Figures $36,37, \& 38$ show the graphical results of Cored Well Number One used as a production set. 


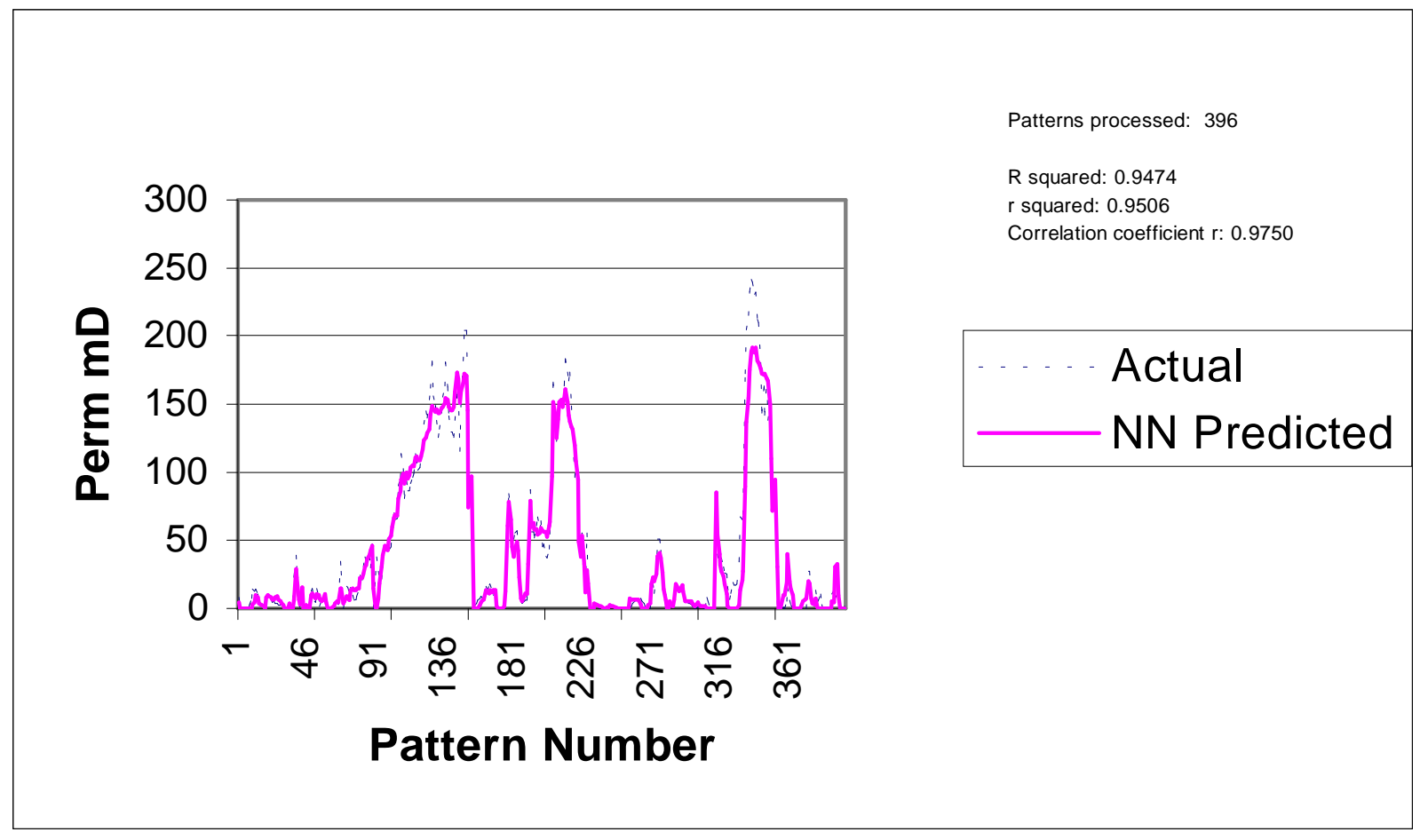

Figure 36 . Cw1 used as a Production Set, Pattern Set

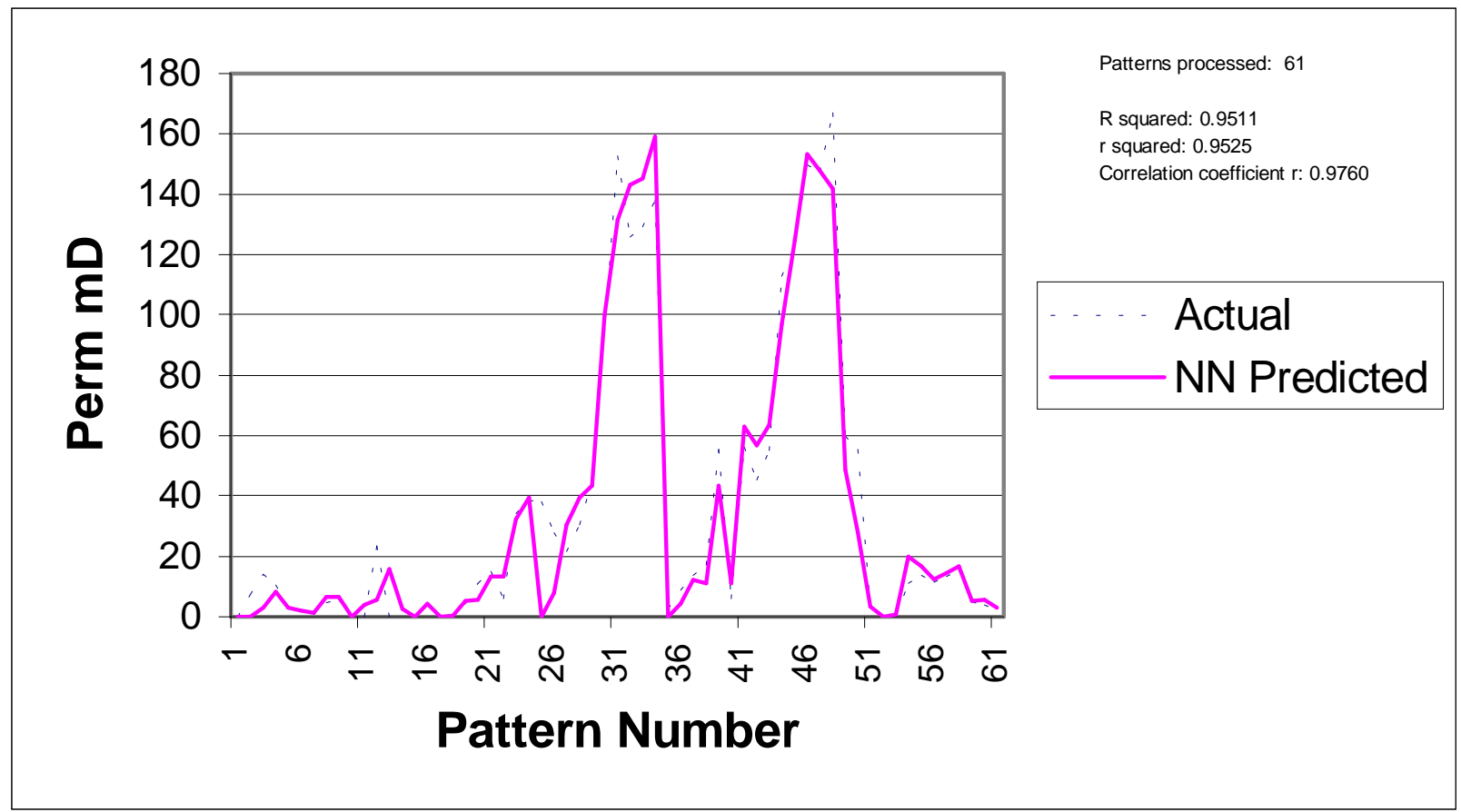

Figure 37. Cw1 used as a Production Set, Test Set 


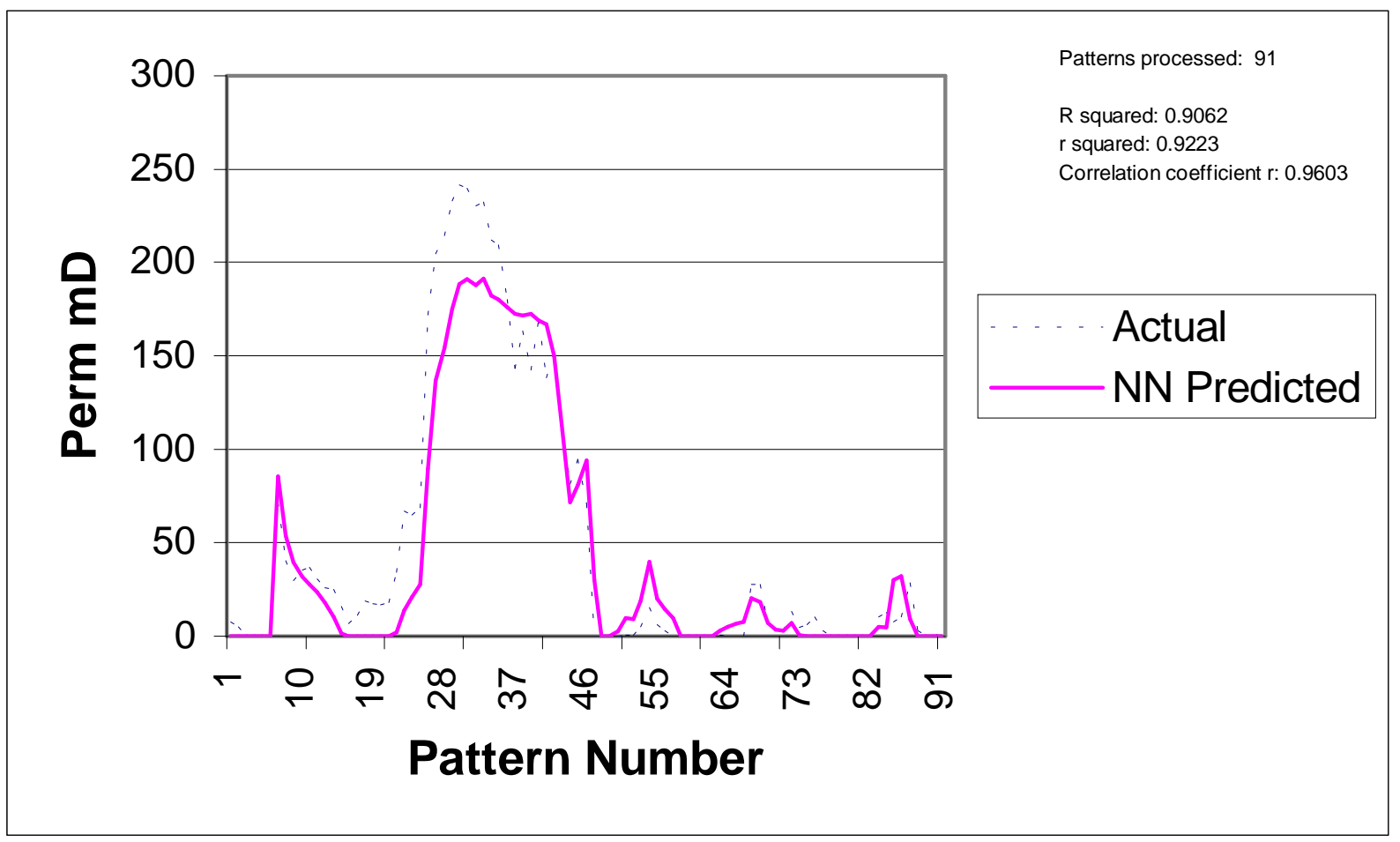

Figure 38. Cw1 used as a Production Set, Production Set 


\subsection{Application of Neural Network Two to Pilot Area:}

With the verification of the Neural Network Two, it finally became possible to achieve the original desired goal of predicting reservoir permeability in the entire pilot area. Having logs available for all of these wells satisfied the input criteria. Again, a DLL was constructed to use in the application process of Neural Network Two. At this point, Neural Network Two was exposed to all of the power area well data in a Microsoft Excel spreadsheet. From this a permeability profile for each was created. These profiles, are shown in the following figures, along with the location of each Pilot Well.

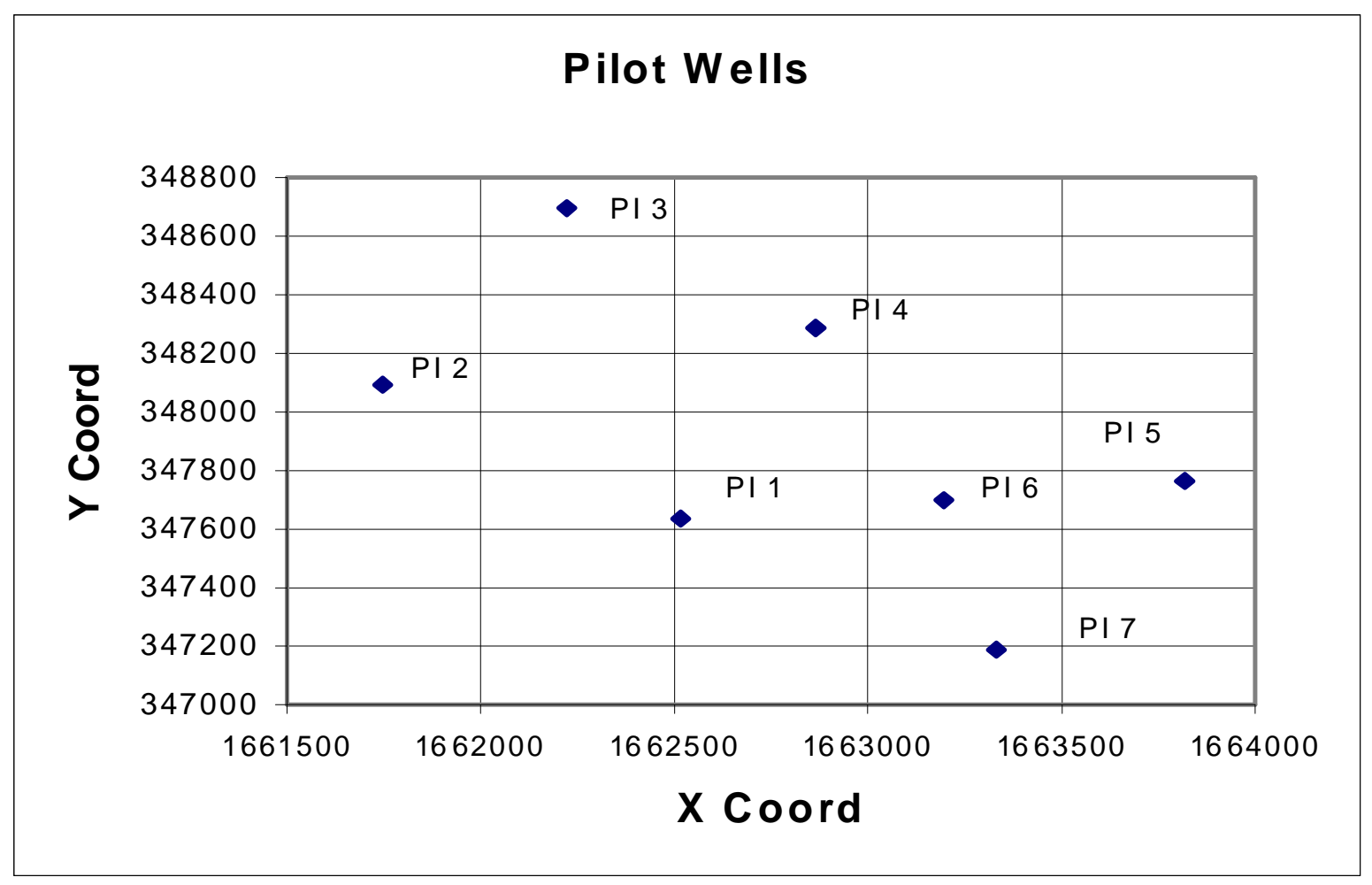

Figure 39. Location of the Pilot Wells 


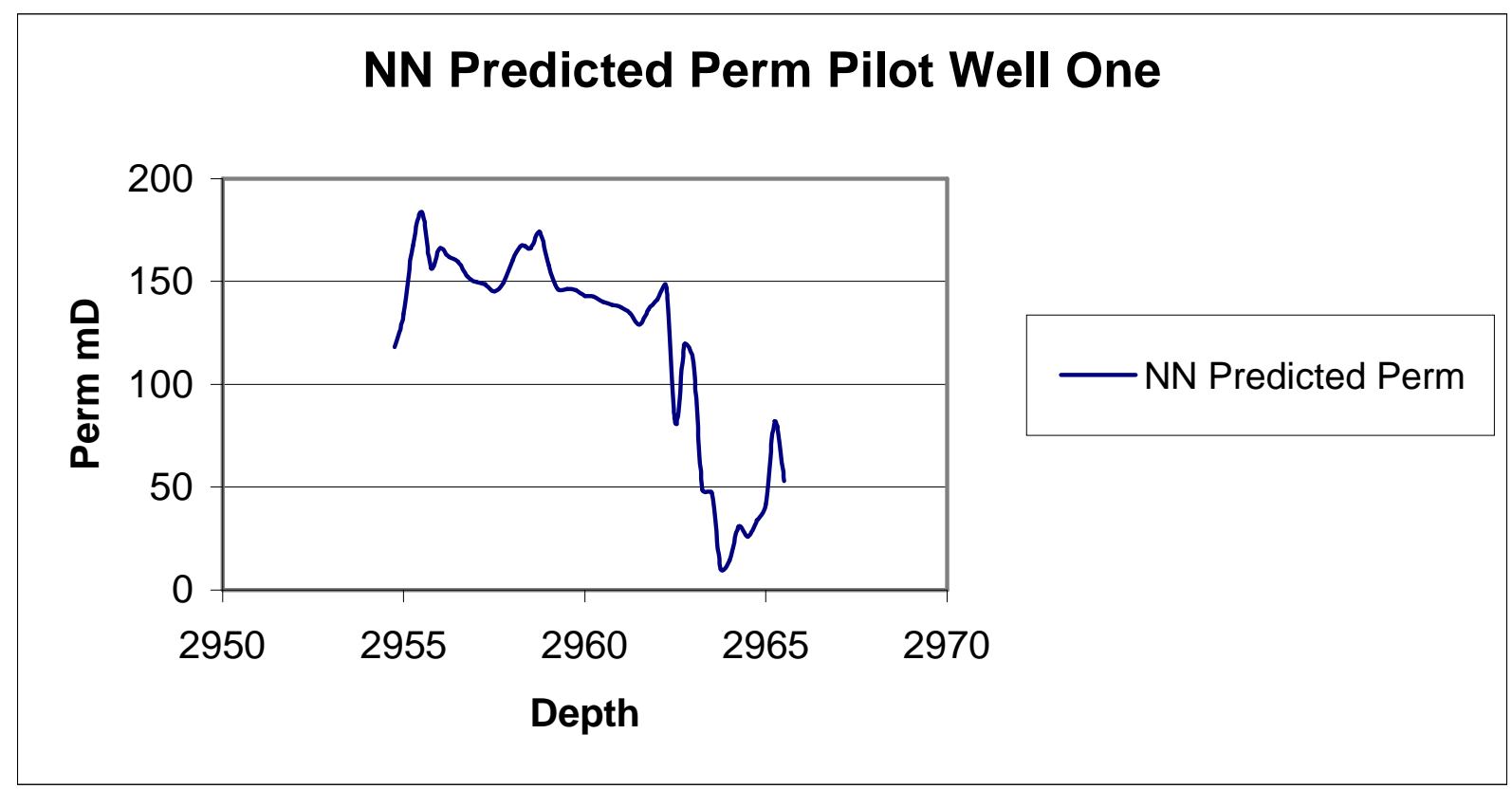

Figure 40. NN Predicted Permeability of Pilot Well 1

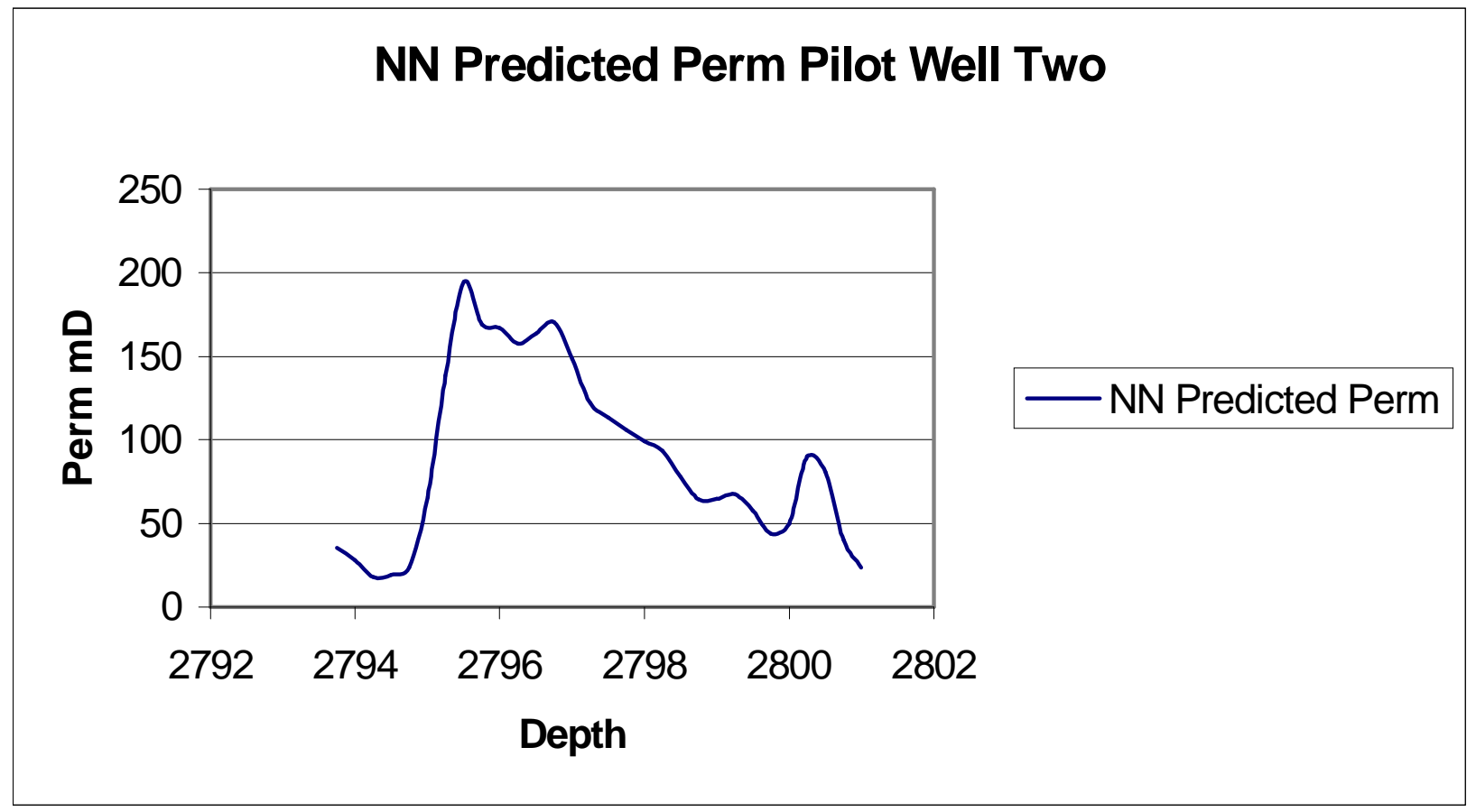

Figure 41. NN Predicted Permeability of Pilot Well 2 


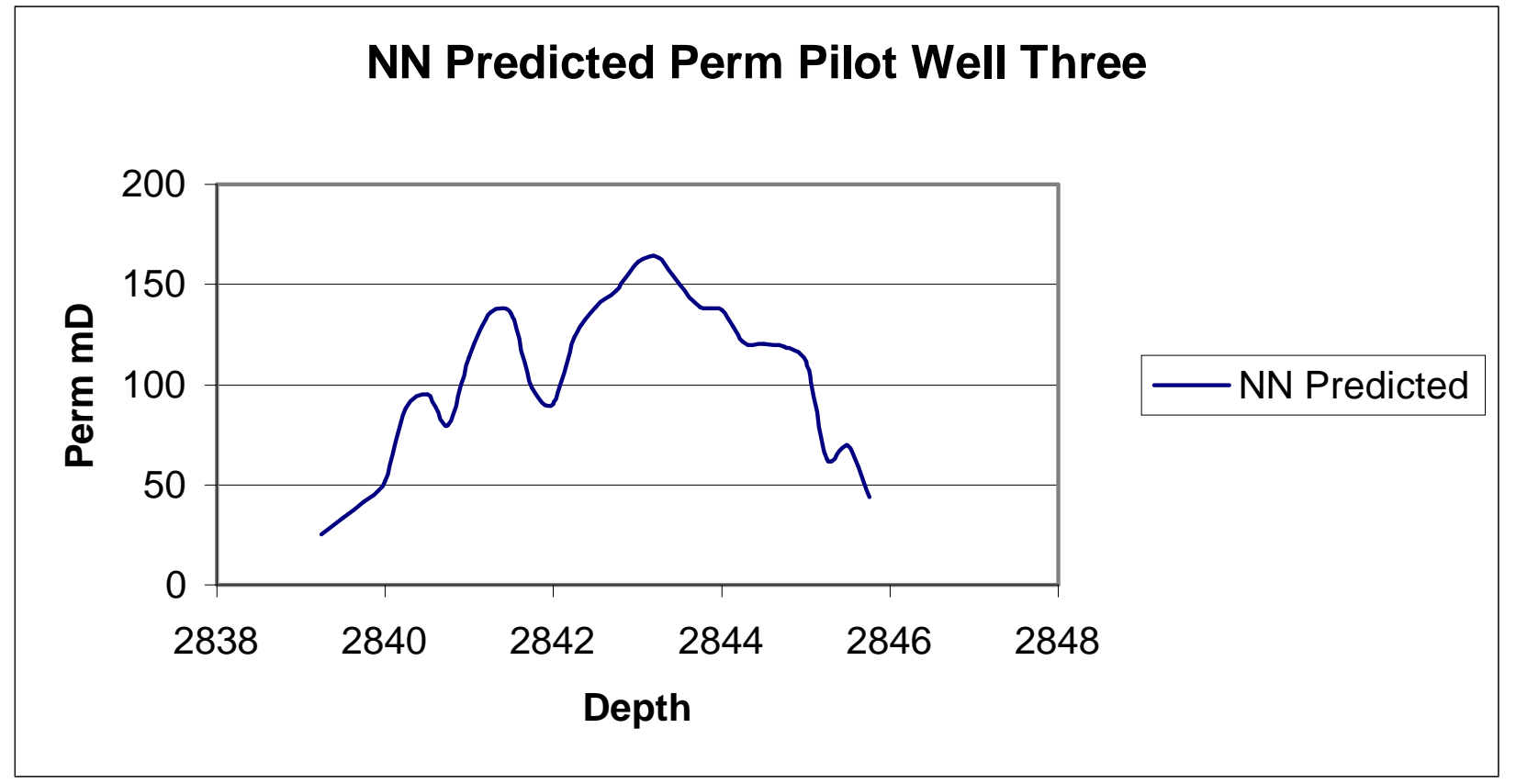

Figure 42. NN Predicted Permeability of Pilot Well 3

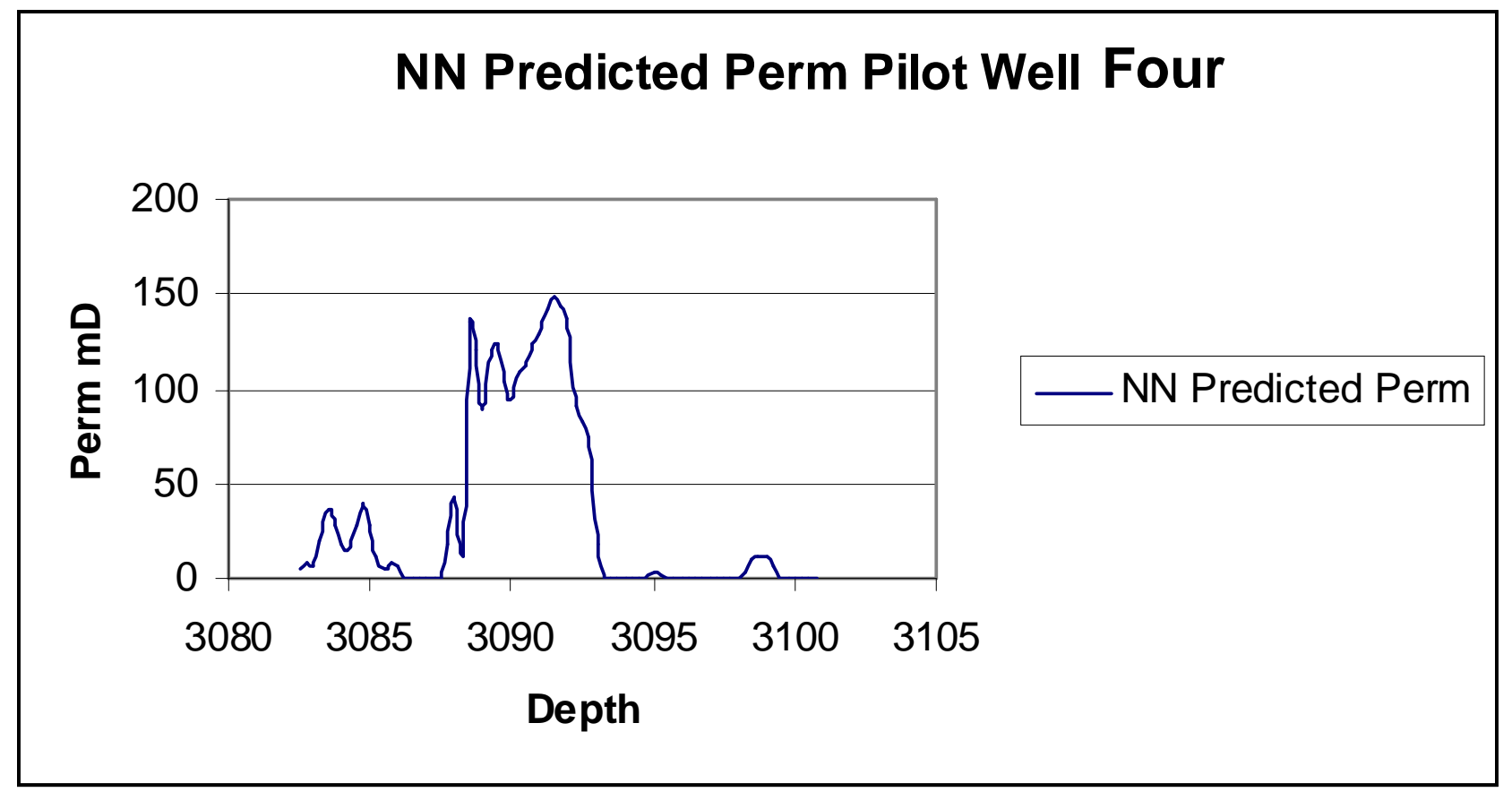

Figure 43. NN Predicted Permeability of Pilot Well 4 


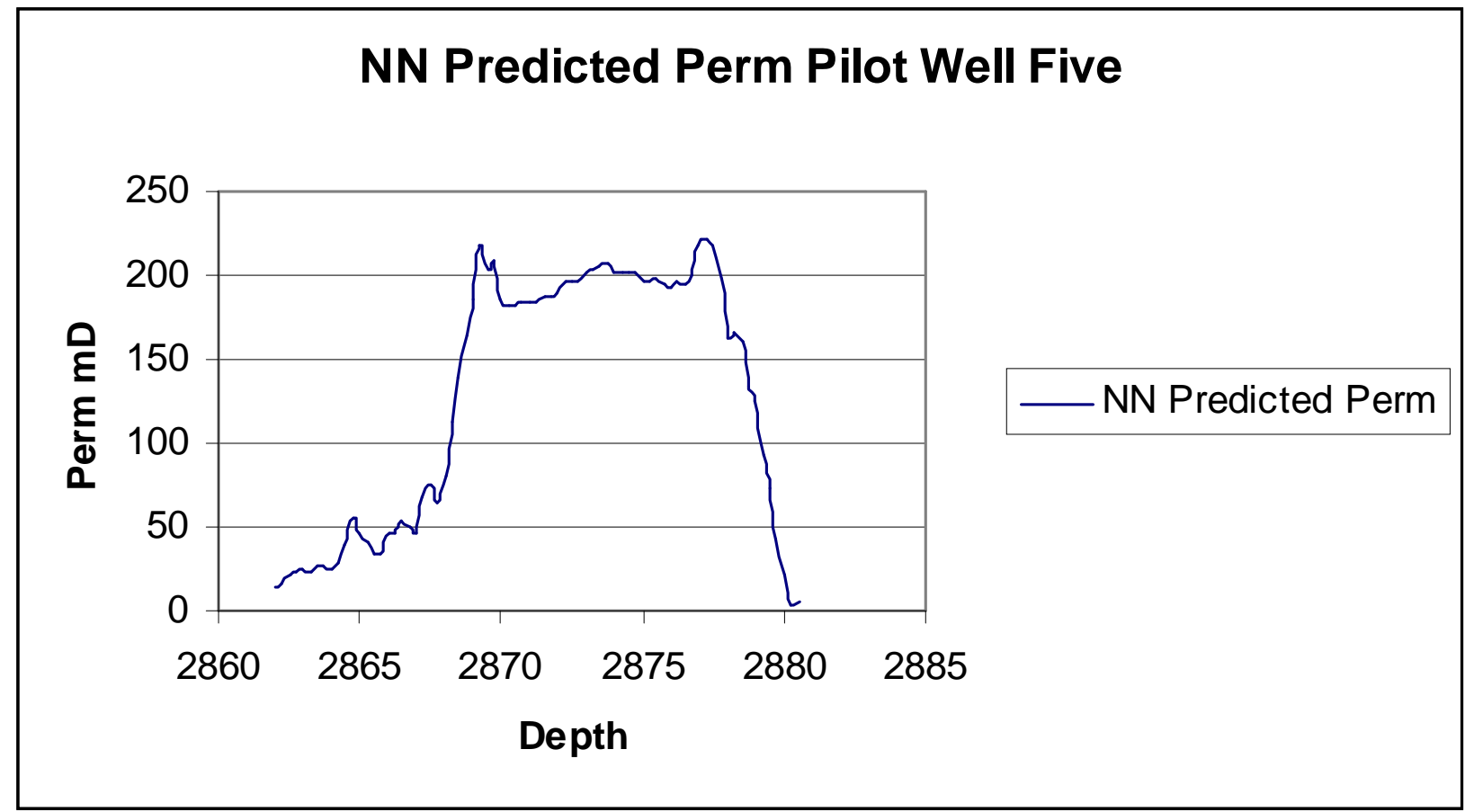

Figure 44. NN Predicted Permeability of Pilot Well 5

\section{NN Predicted Perm Pilot Well Six}

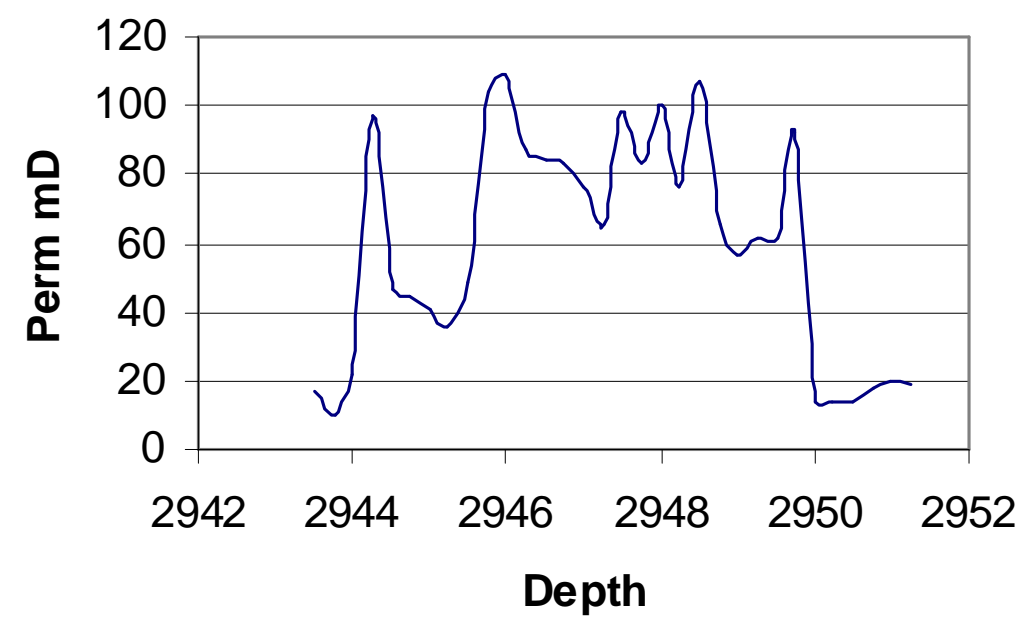

Figure 45. NN Predicted Permeability of Pilot Well 6 


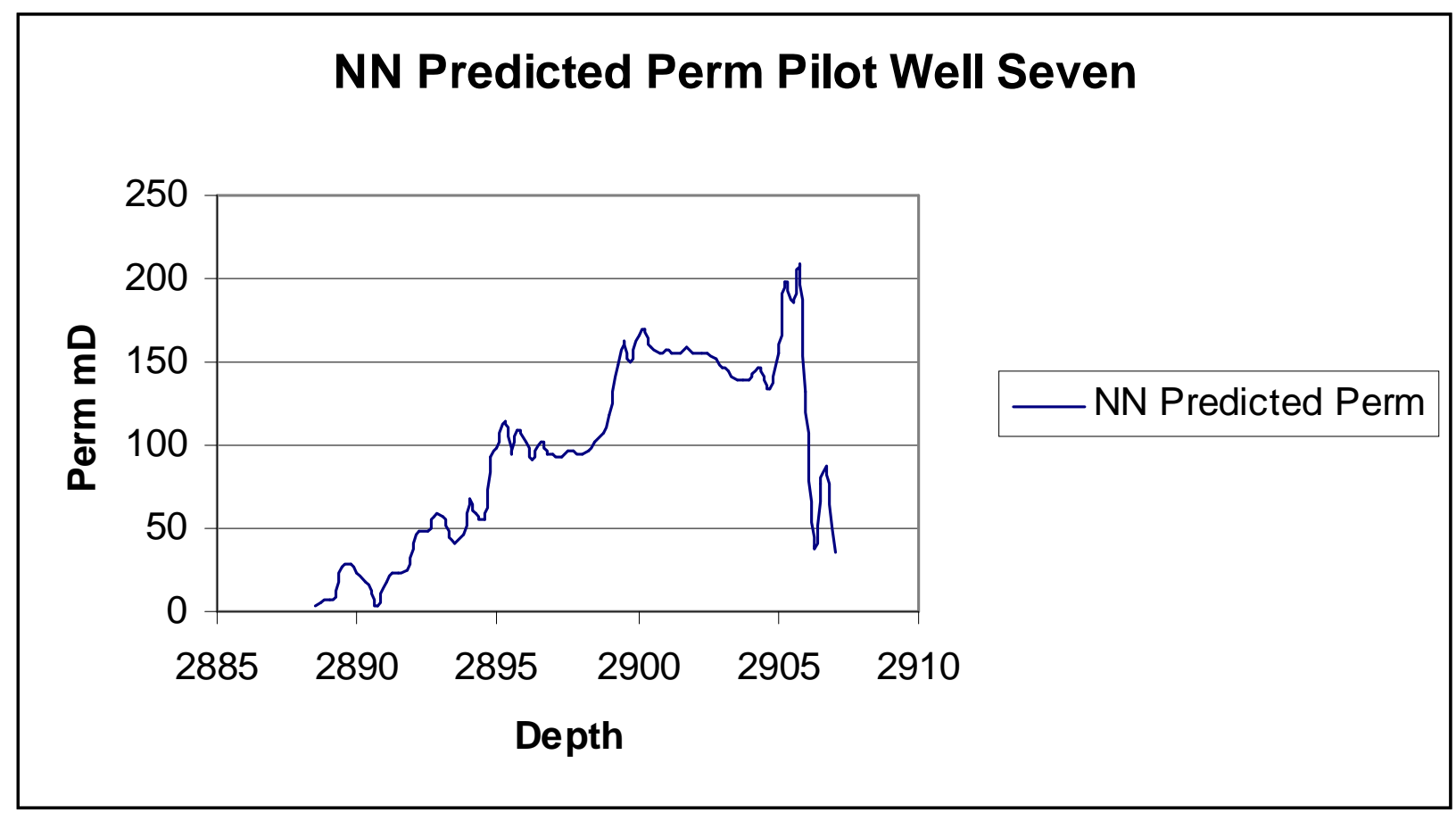

Figure 46. NN Predicted Permeability of Pilot Well 7

The raw data for each pilot well can be found in Appendix XV. A detailed look at these profiles can provide valuable insight into the permeability trends of each of these pilot wells. One may also get a good view of depths and thicknesses associated with the pay sands, by charting depths corresponding to areas of predicted high permeability. 


\section{Chapter Five: Conclusions and Recommendations}

\subsection{Conclusions:}

As mentioned before it was essential that each artificial neural network constructed be verified in order to be safely used to predict permeability in wells or core permeability from mini-permeability at depths not included in the network's training. These were satisfactorily done and the permeabilities predicted for the pilot area are considered realistic and representative of the major trends.

Also, with respect to the Neural Network One and the minipermeameter readings, a direct relationship to reservoir permeability was never established. Only by having core permeabilities and the application of artificial neural networks was a good correlation created. This suggests a more complex relationship than was hypothesized before the study began.

Reasons for this mistake may be several unknowns about the inner workings of the minipermeameter itself, and the processes used to achieve the readings. Another point of concern with respect to the readings of the minipermeameter, is the time frame in which they were taken. This time frame causes concern because the minipermeameter readings were taken several years after the core analysis. This poses the questions; how were the cores cared for in this space of time, and how much did their quality degrade if any? Each of these could be the cause for the lower values of mini-permeability, while retaining the basic trends of the core analysis.

The minipermeameter readings do indicate a type of permeability that is comparable to the reservoir permeability as demonstrated by the success of Neural Network One with the inclusion of the log data. The key point being that this success 
was only achieved with the reservoir properties included. The construction and verification of Neural Network One, with an R squared value of .97, makes the usage of mini-permeability data feasible in other areas, with the addition of log data and limited core analysis of several reasonably representative wells. Establishing this connection between the two permeability readings through this network constituted the major discovery in this study. The construction of the Neural Network Two using log data with core permeability as the desired output simply allowed for an application of that discovery, since this process has been substantially proven in previous research. This application however, is capable of giving useful insight into the pilot area with the potential to be used on a wider scale with the availability of more well cores and/or using the generated values in a reservoir simulator program.

Artificial neural networks, specifically the Back-propagation architectures, again proved their ability to be beneficial in the petroleum industry. They have consistently been able to add insight and information into questions/problems that are difficult to solve with traditional petroleum engineering techniques. This case provides a good example, because of the heterogeneity of the Stringtown field and the unknown relationship between mini-permeability and core permeability. 


\subsection{Recommendations}

One of the concerns mentioned earlier, limited knowledge about the minipermeameter, stands out as a significant area of possible improvement. The readings produced by the minipermeameter had to be taken at face value. In depth knowledge of the inner workings and procedures used in its measurement could help one to recognized faulted readings and allow for more manual manipulation of the data.

Another drawback of the study was the small number of cored wells with minipermeameter data. In the area of artificial neural networks, the more data that can be used in training, the better the prediction capabilities of the network. More cored and analyzed wells may have helped both networks to become more viable and also applicable on a wider scale, possibly the entire field. Taking this undersized training set into effect, it was determined that both networks would only predict well on the pilot area and wells very near to it .

Lastly, the possibility remains open for the use of different architectures and structures to improve the results. Several variances were tried, but even more not attempted. The addition of new inputs could also expand the capabilities of the network. Some inputs suggested in other research are neutron porosity and spontaneous potential logs. Continued interest in this area by the petroleum industry should help to uncover the most beneficial combinations of architectures and inputs. 
Appendix 


\section{Appendix I - Log Comparisons for Cored Wells}

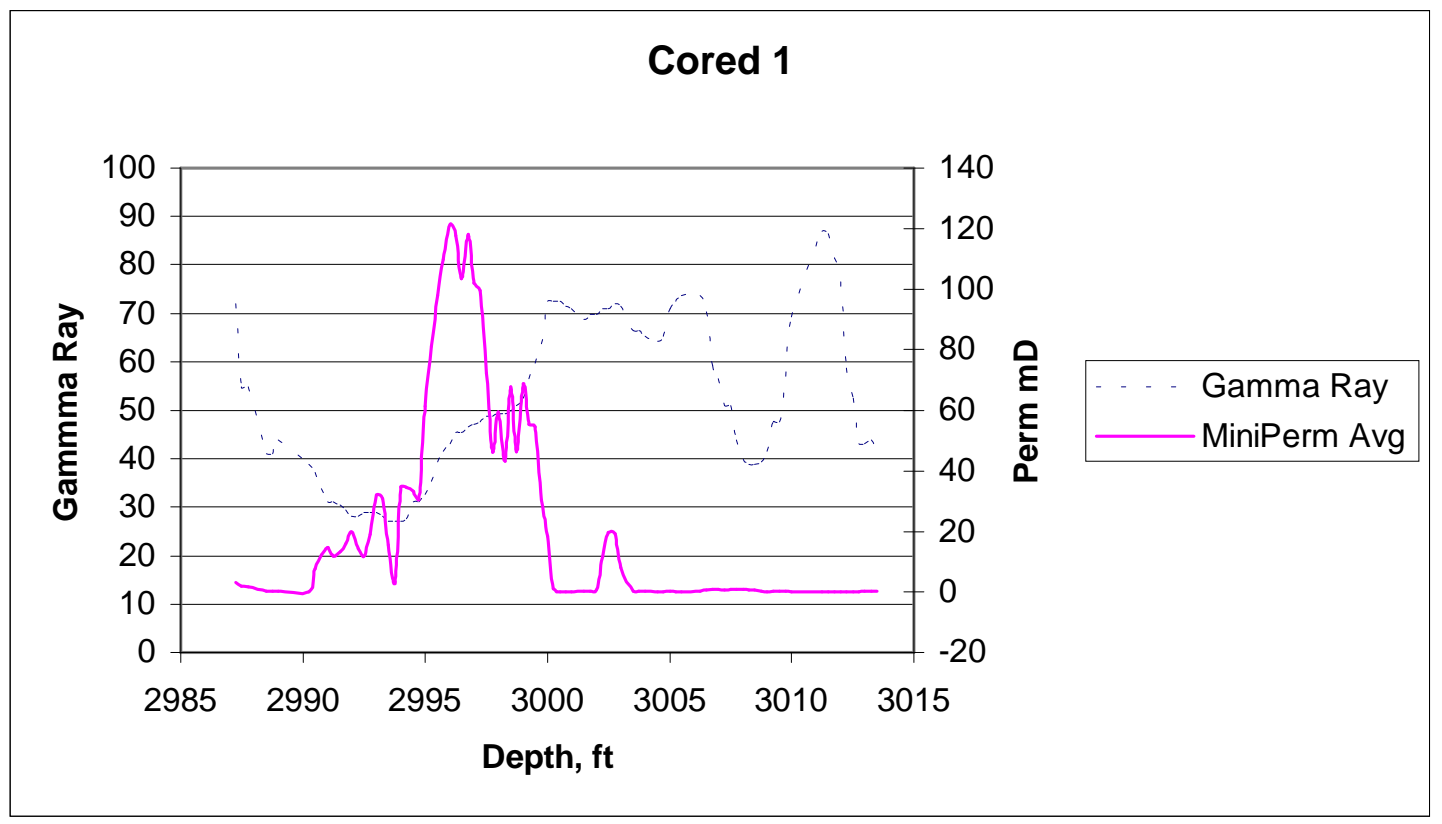

Figure A1. Gamma Ray Verses Minipermeameter Values for $\mathrm{Cw}_{1}$

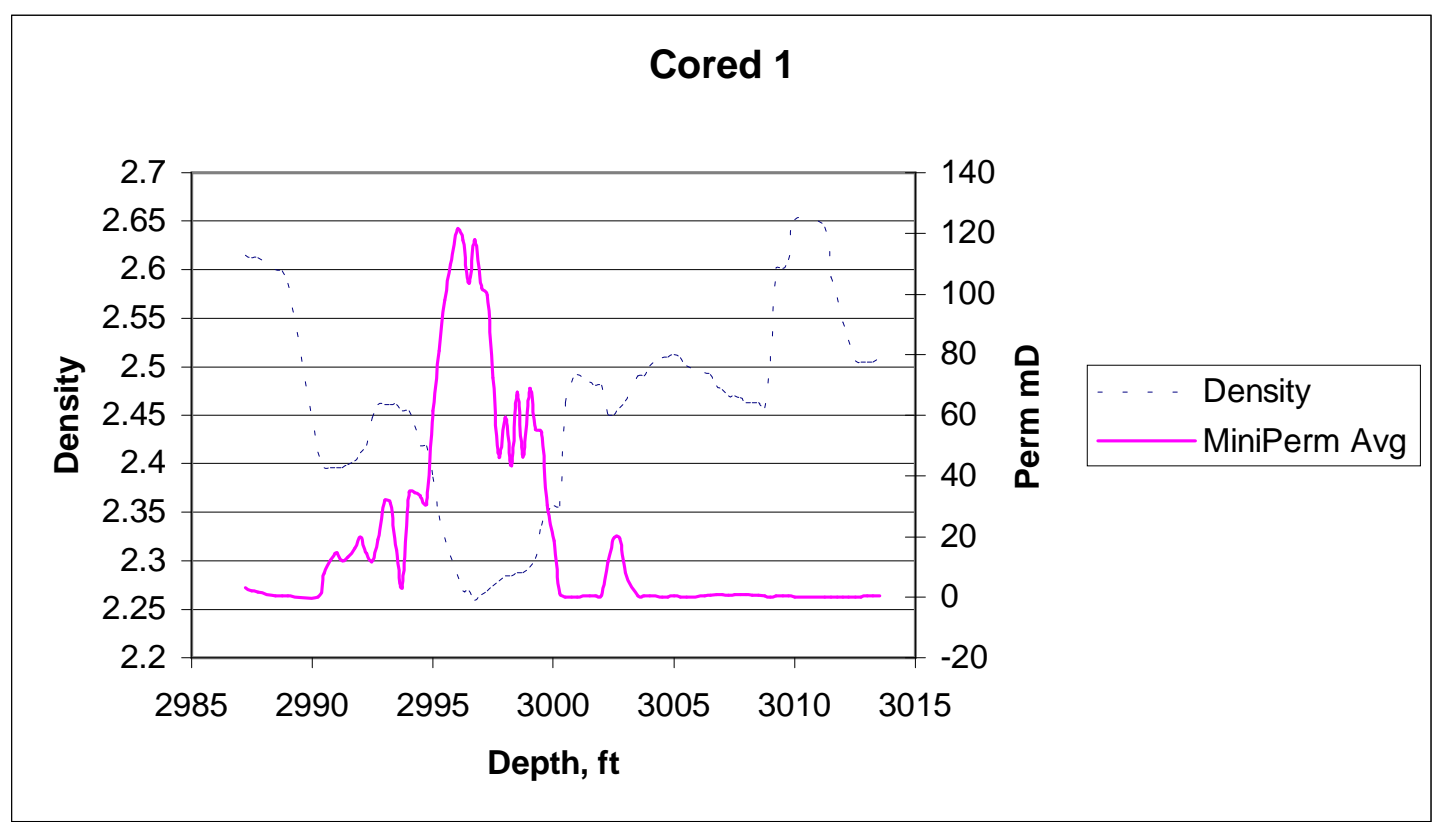

Figure A2. Density Verses Minipermeameter Values for Cw 1 


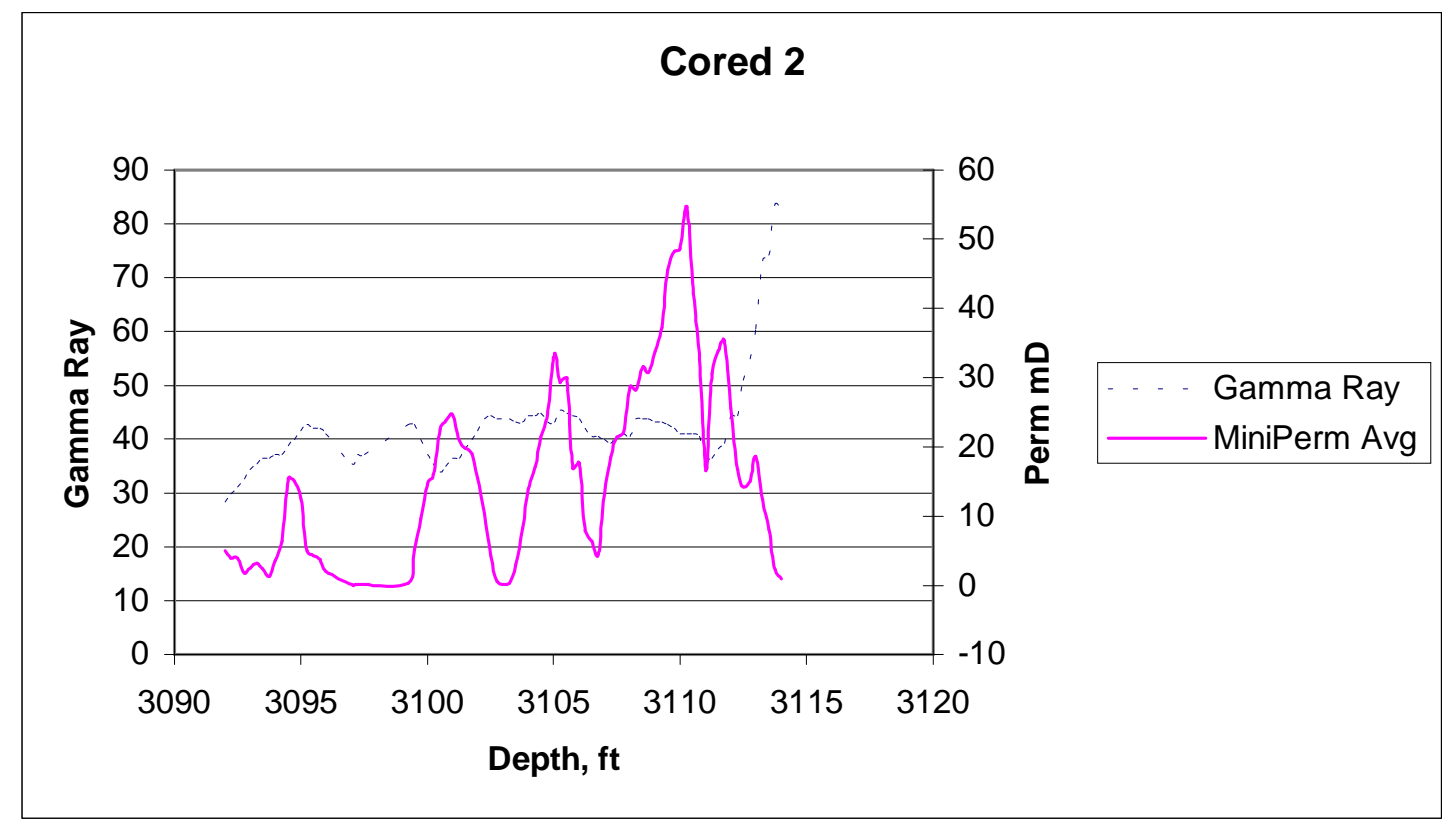

Figure A3. Gamma Ray Verses Minipermeameter Values for Cw 2

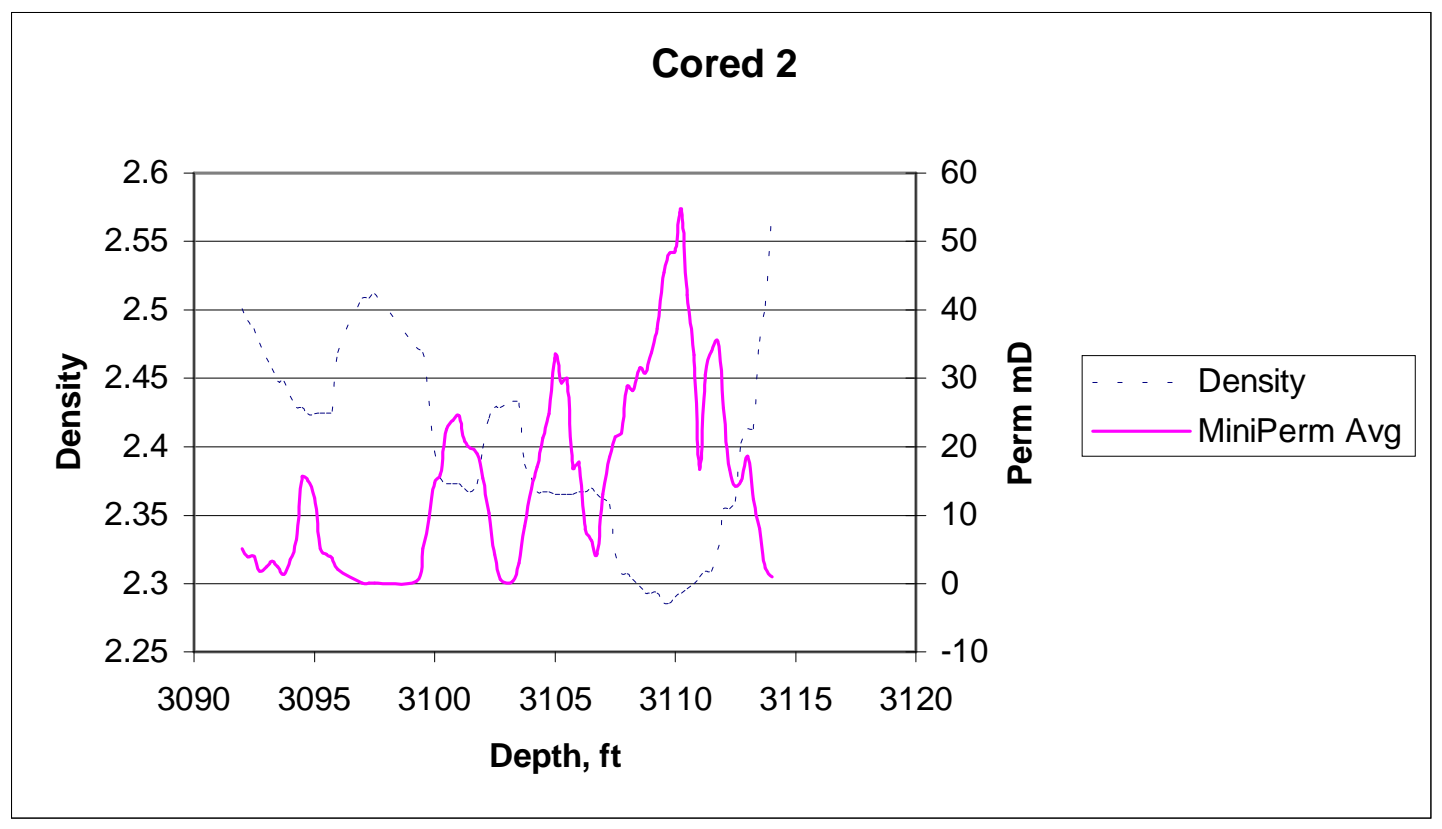

Figure A4 . Density Verses Minipermeameter Values for Cw 2 


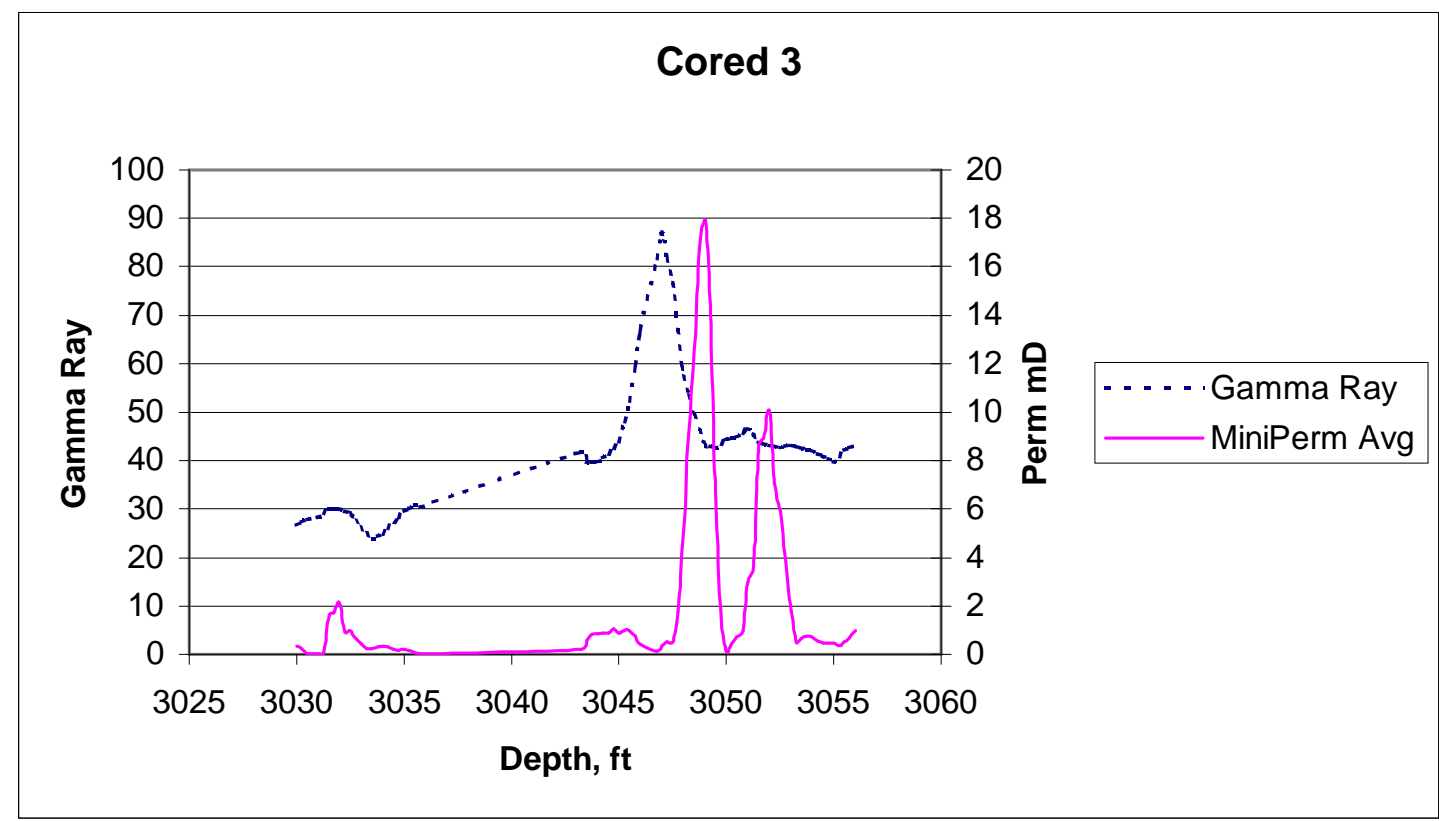

Figure A5 . Gamma Ray Verses Minipermeameter Values for Cw3

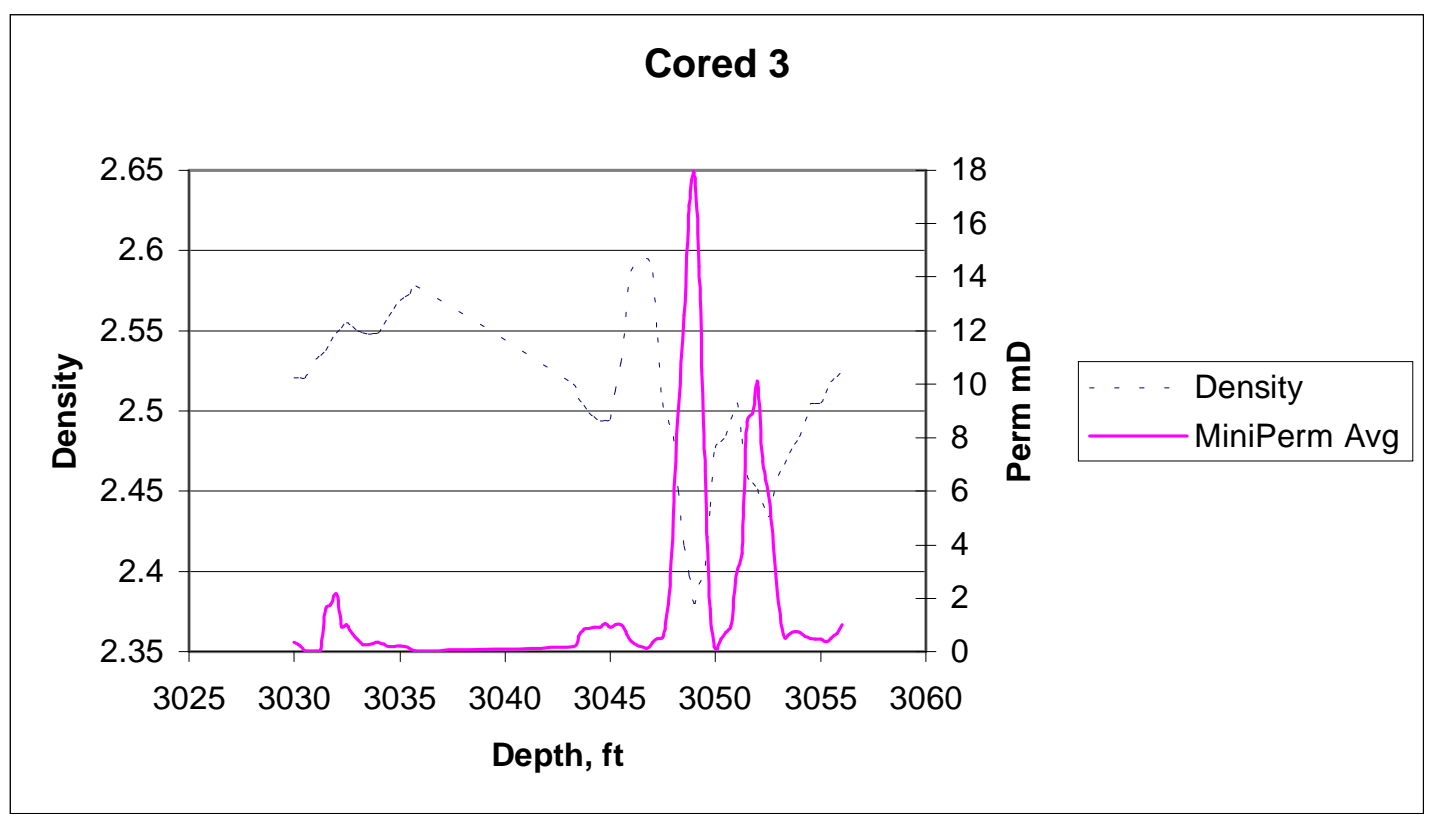

Figure A6. Density Verses Minipermeameter Values for Cw 3 


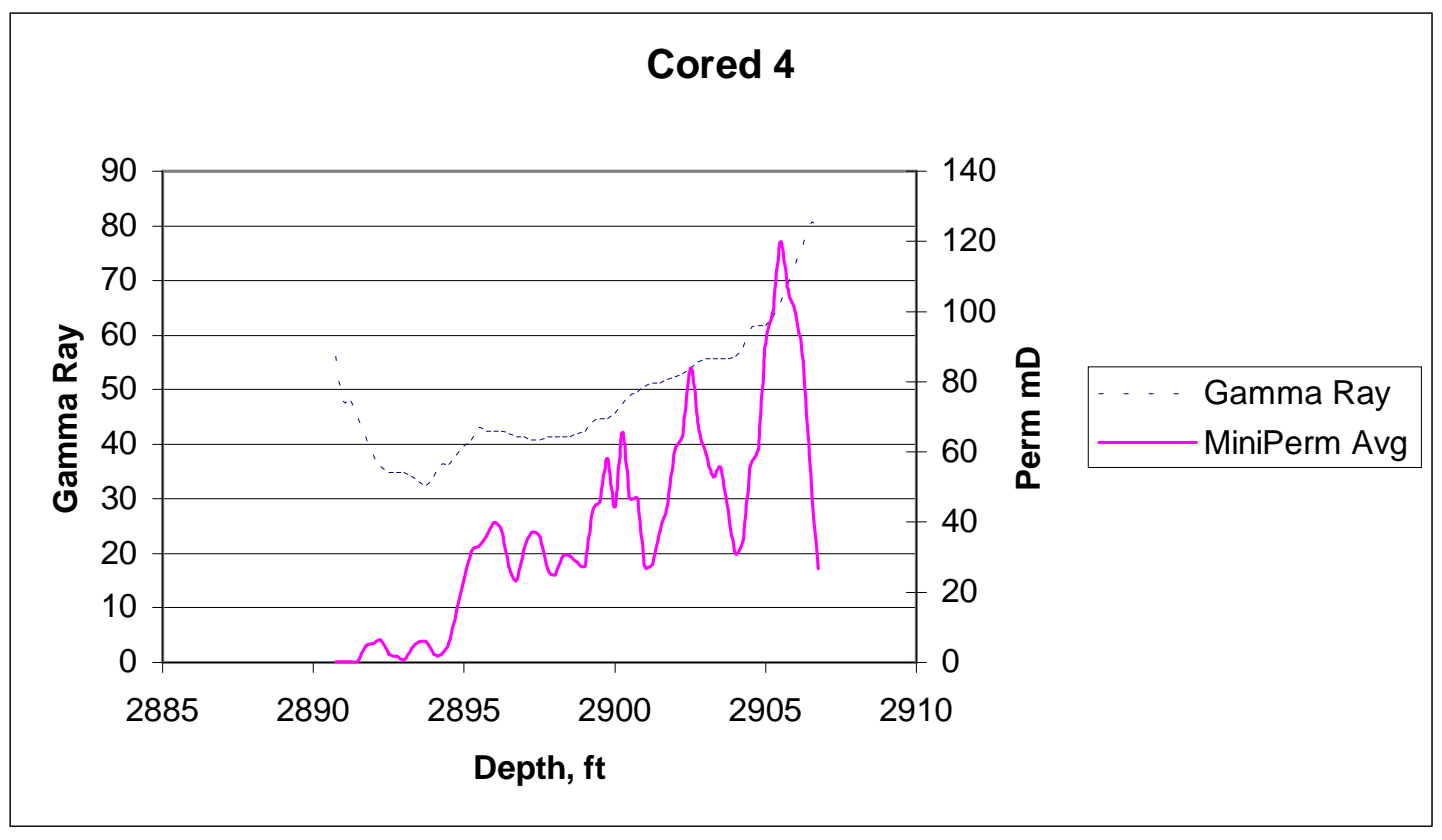

Figure A7. Gamma Ray Verses Minipermeameter Values for Cw4

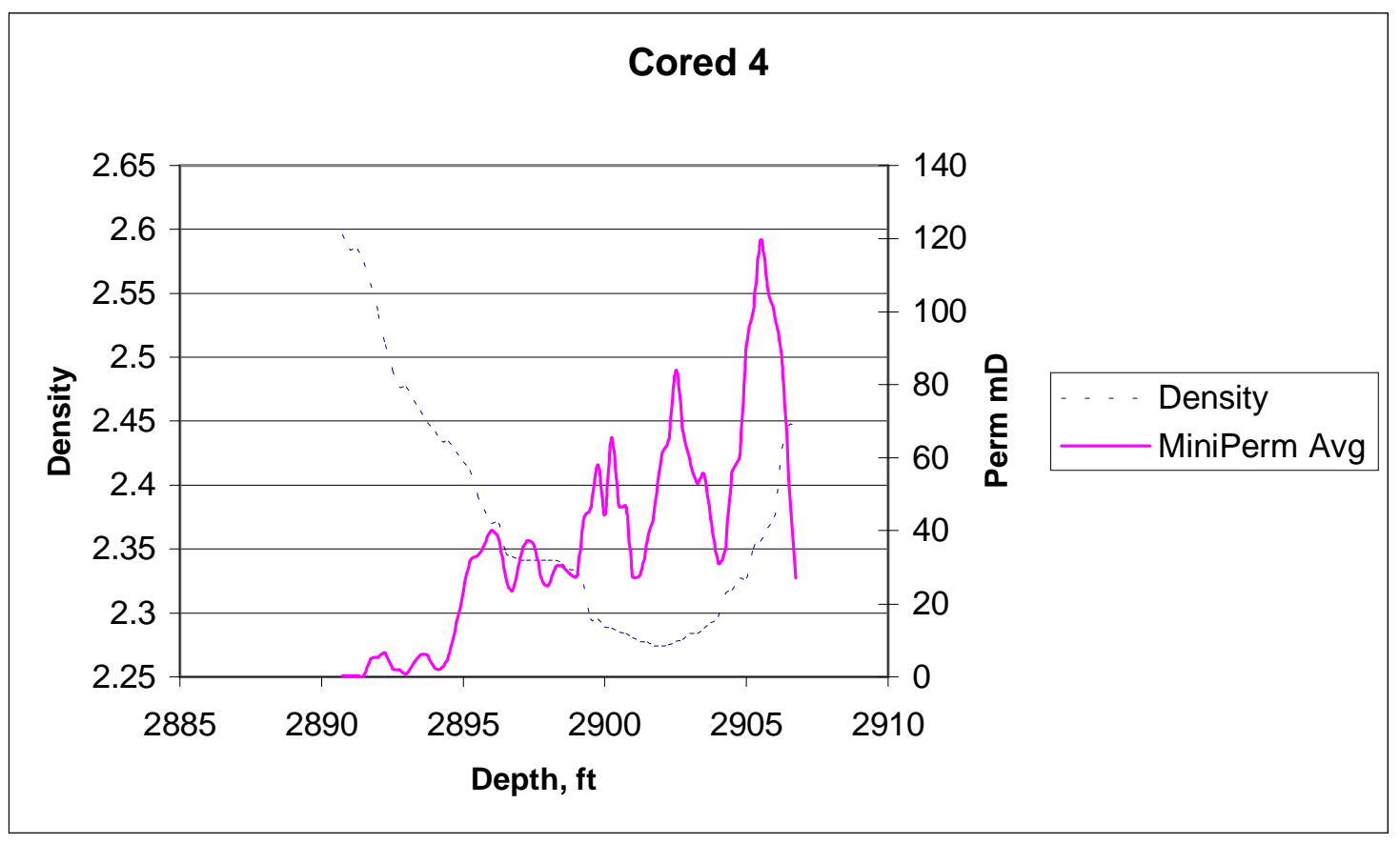

Figure A8 . Density Verses Minipermeameter Values for Cw 4 


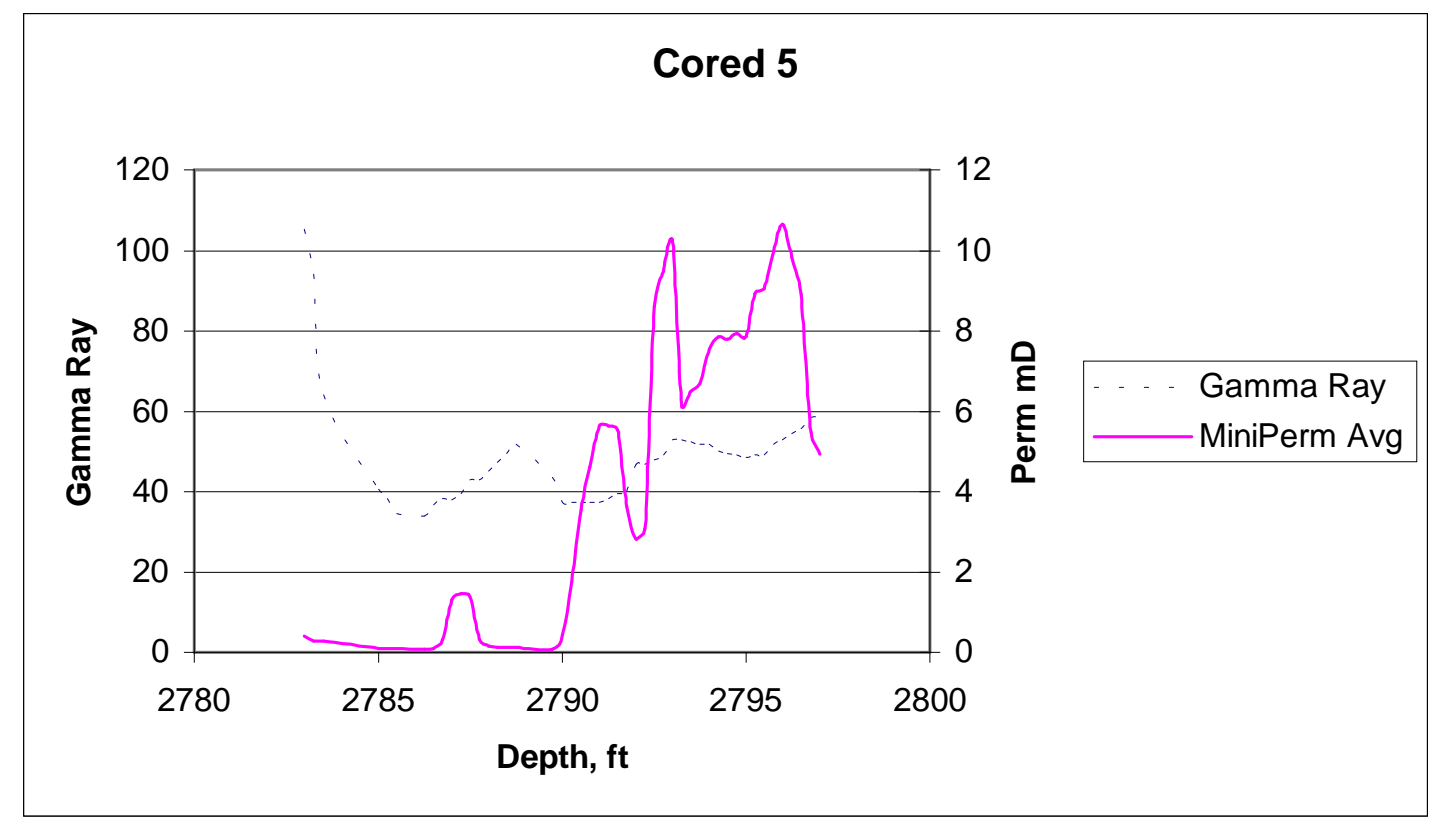

Figure A9 . Gamma Ray Verses Minipermeameter Values for Cw5

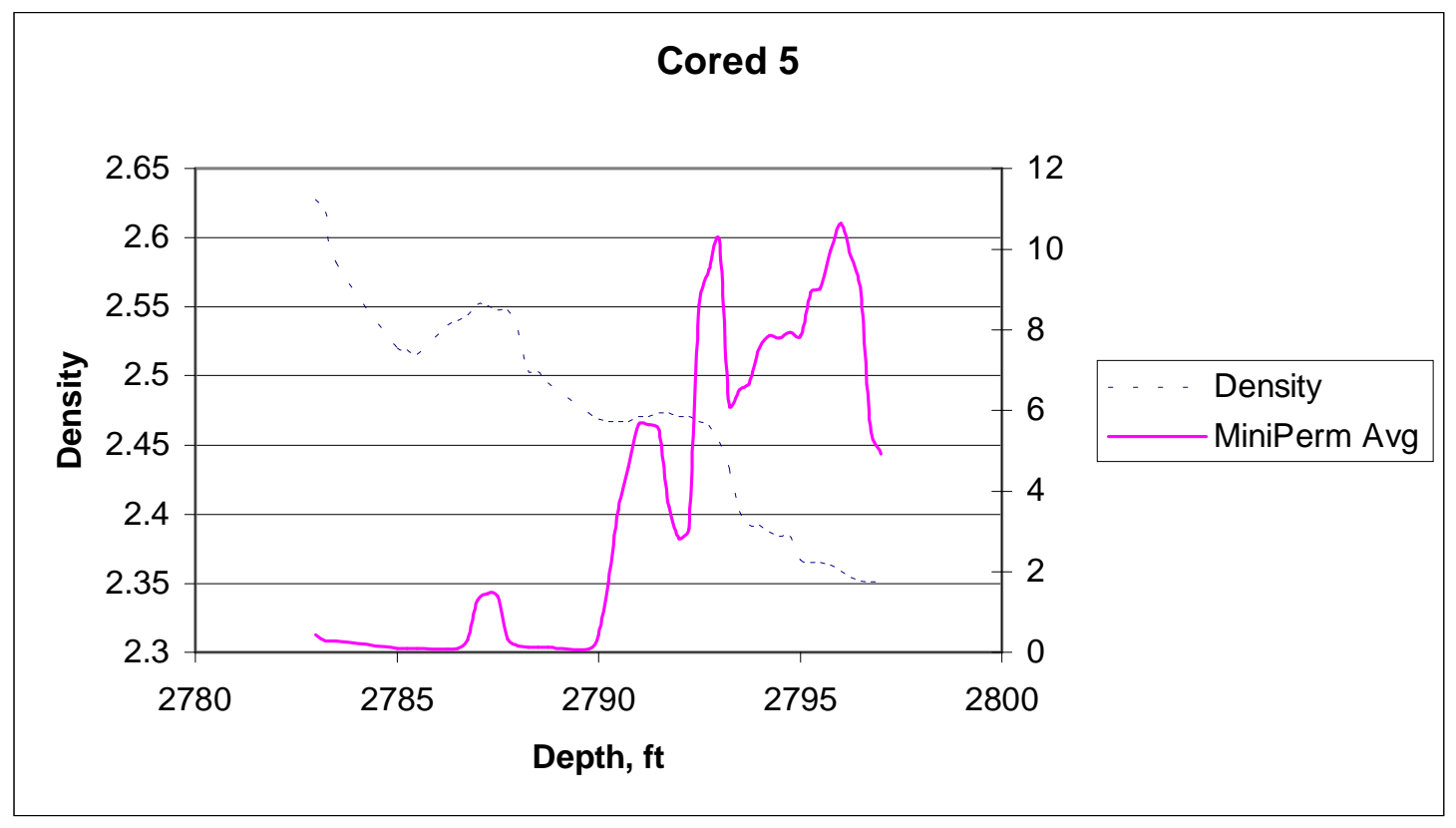

Figure A10. Density Verses Minipermeameter Values for Cw 5 
Appendix II - Minipermeameter Averages for each cored well

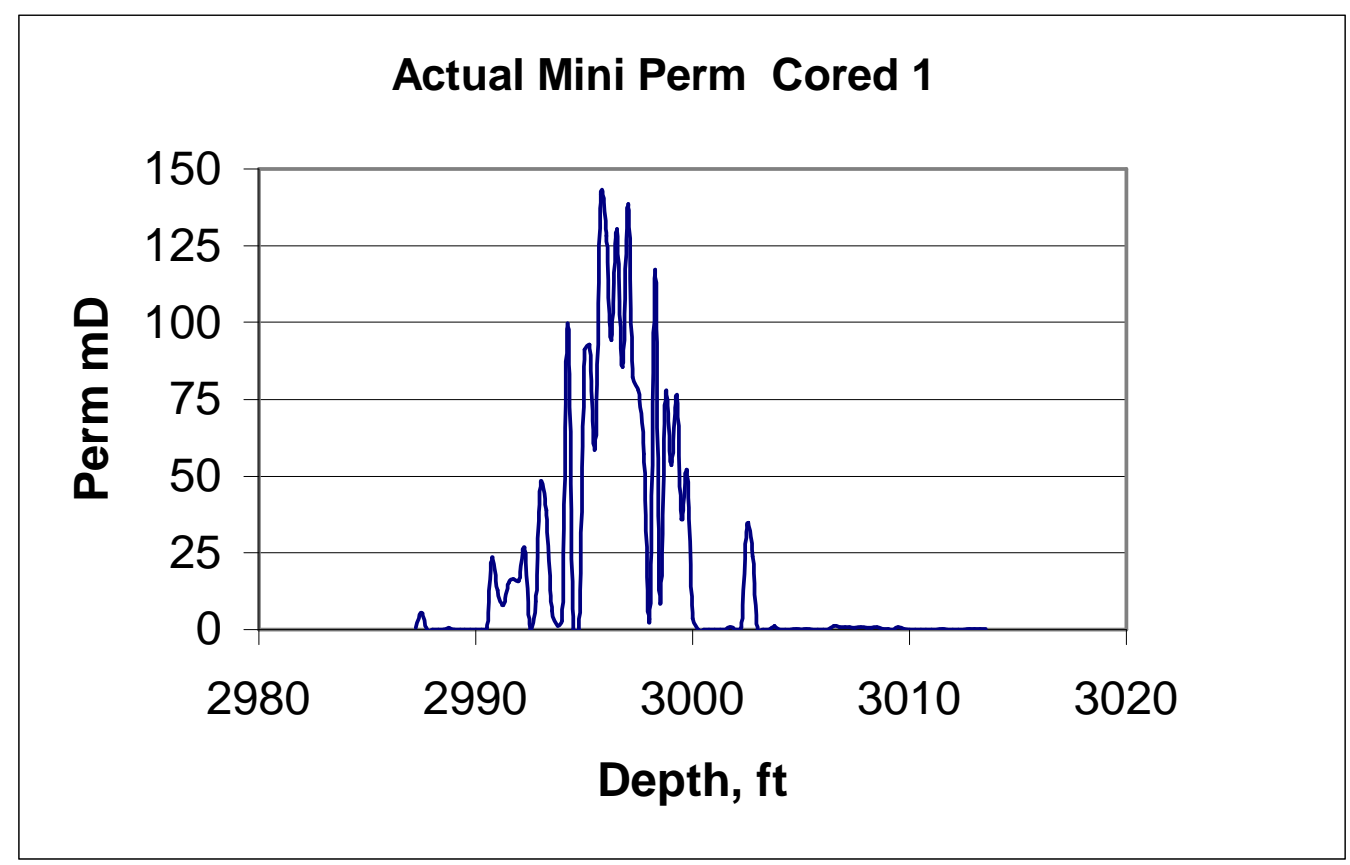

Figure A11. Actual Minipermeameter Values for Cw1

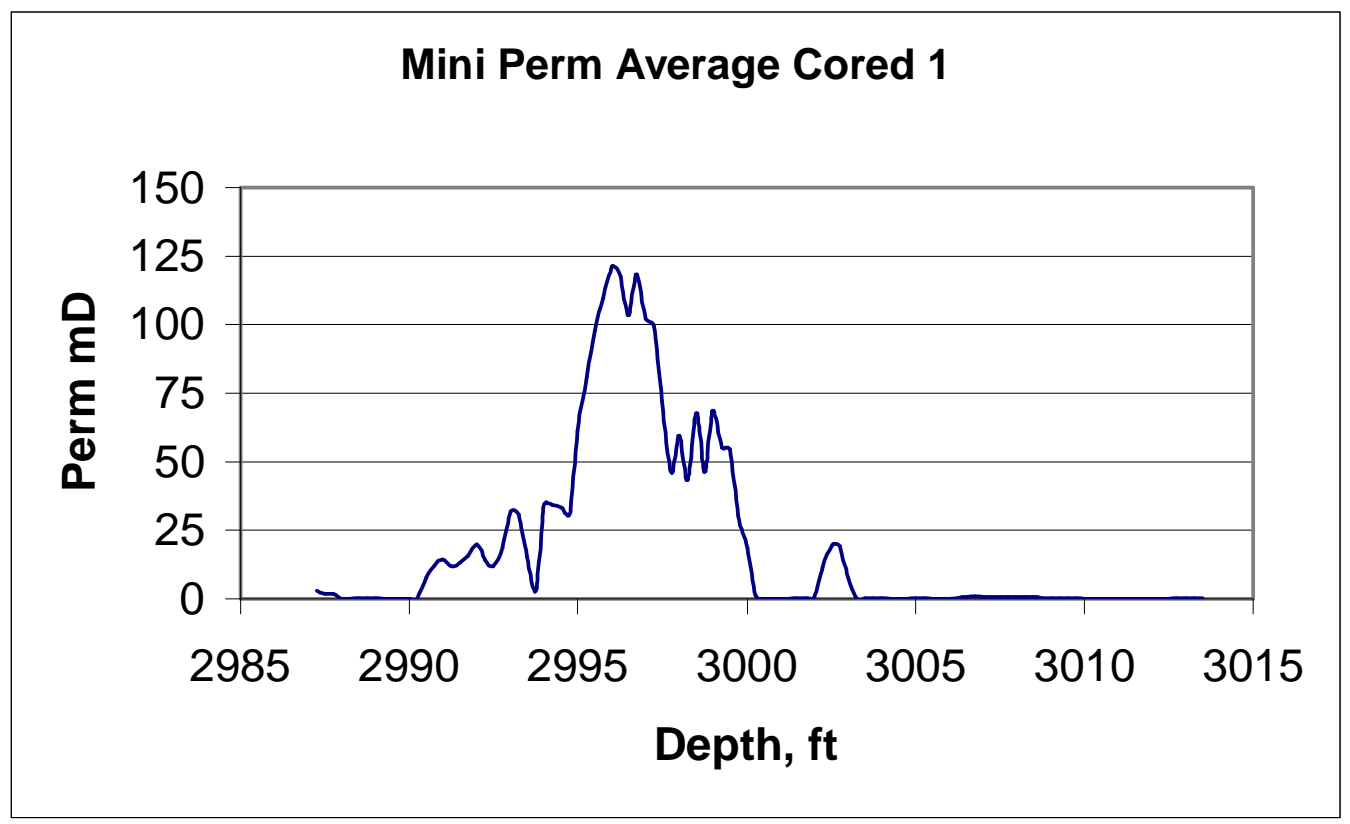

Figure A12. Averaged Minipermeameter Values for Cw1 

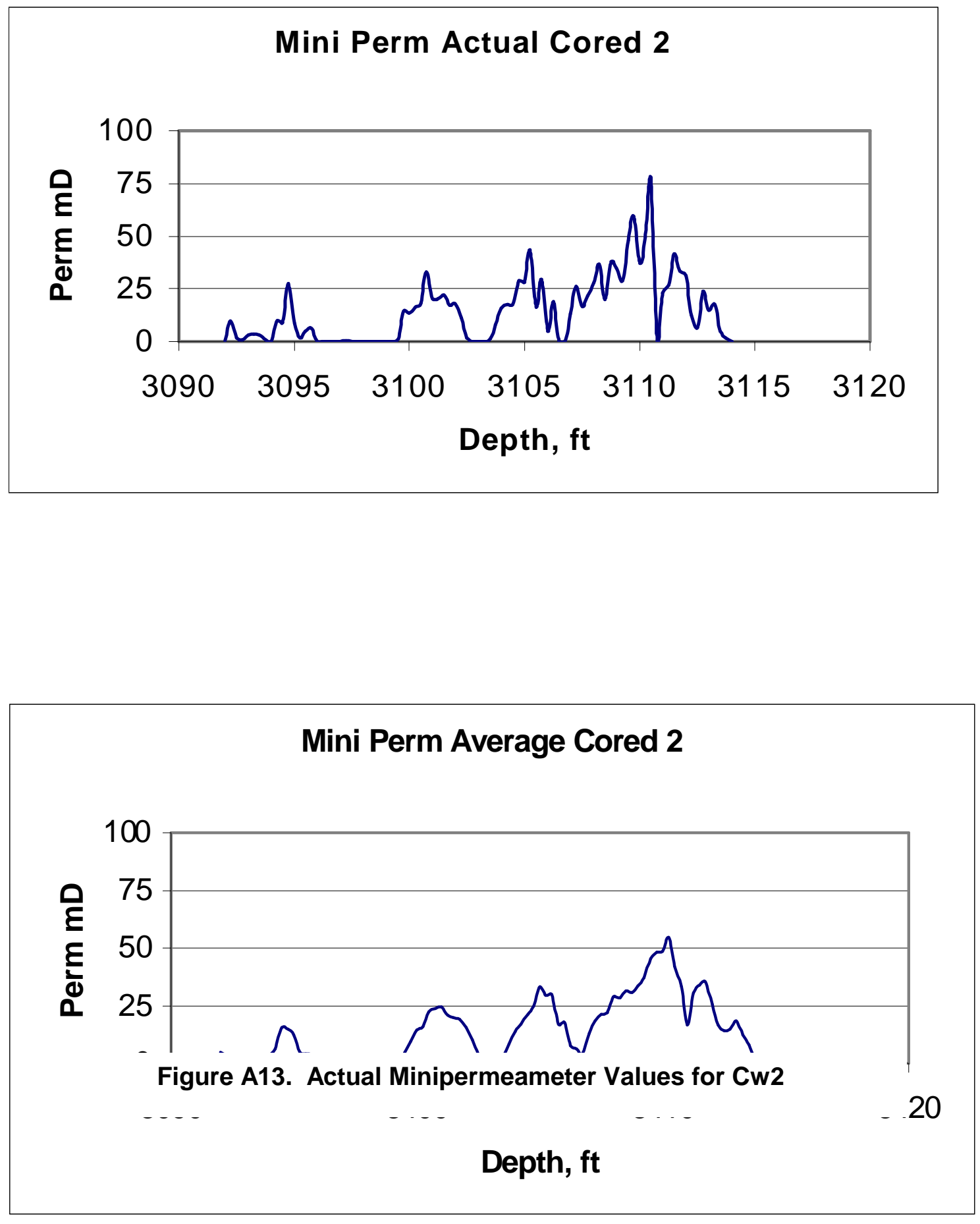

Figure A14. Averaged Minipermeameter Values for Cw2 


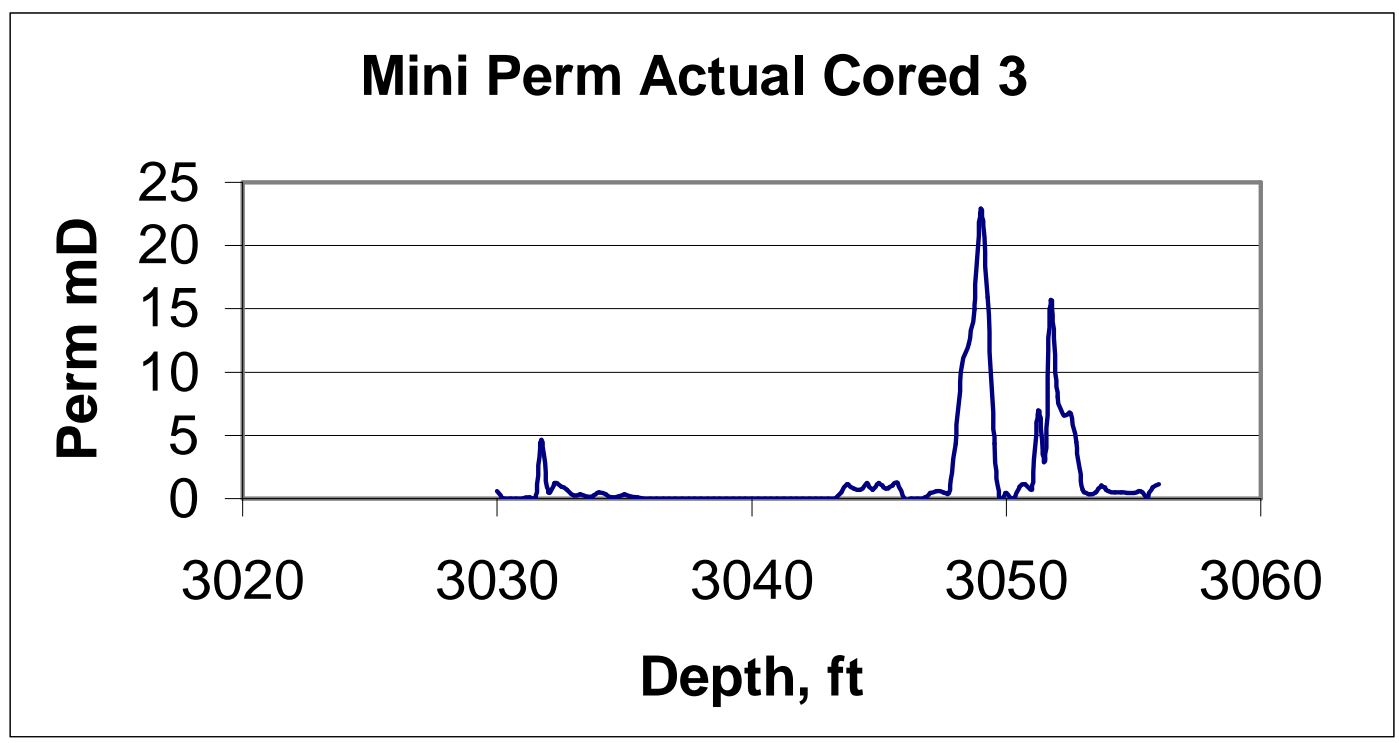

Figure A15. Actual Minipermeameter Values for Cw3

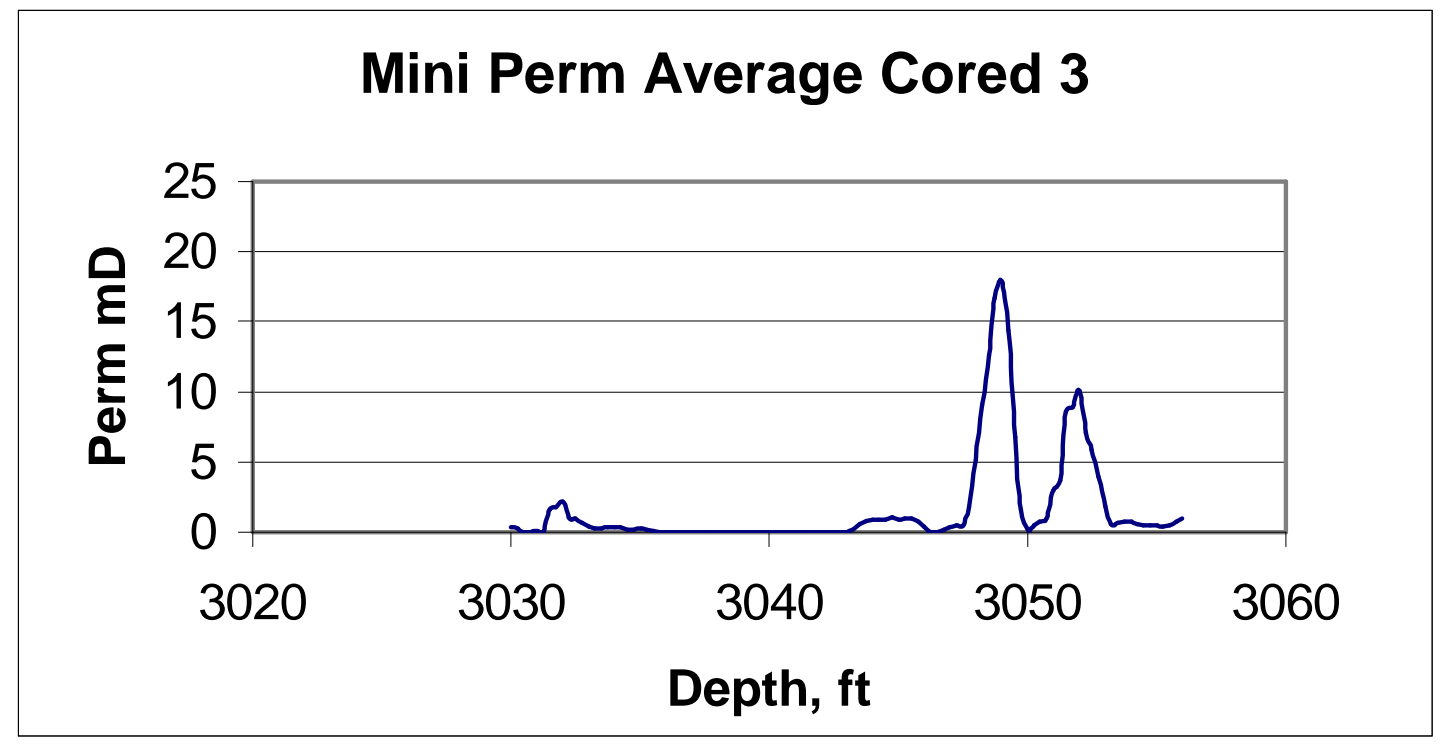

Figure A16. Averaged Minipermeameter Values for Cw3 


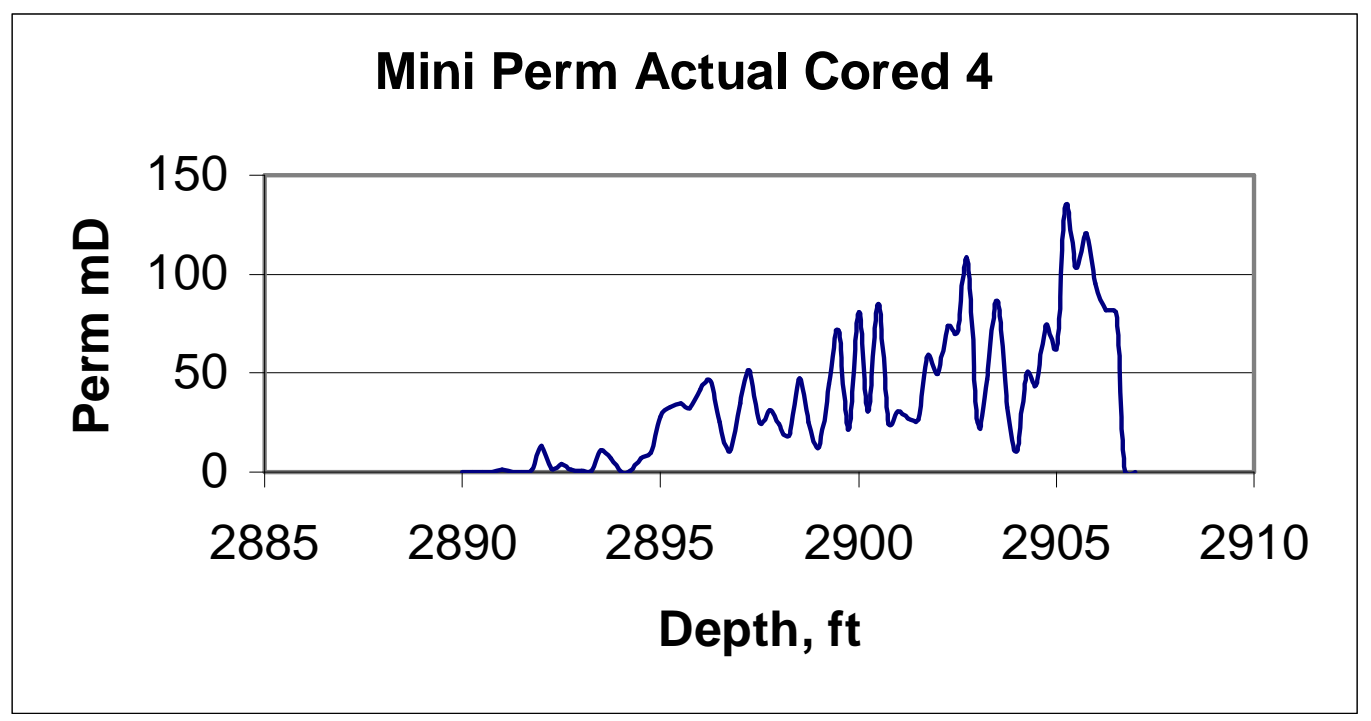

Figure A17. Actual Minipermeameter Values for Cw4

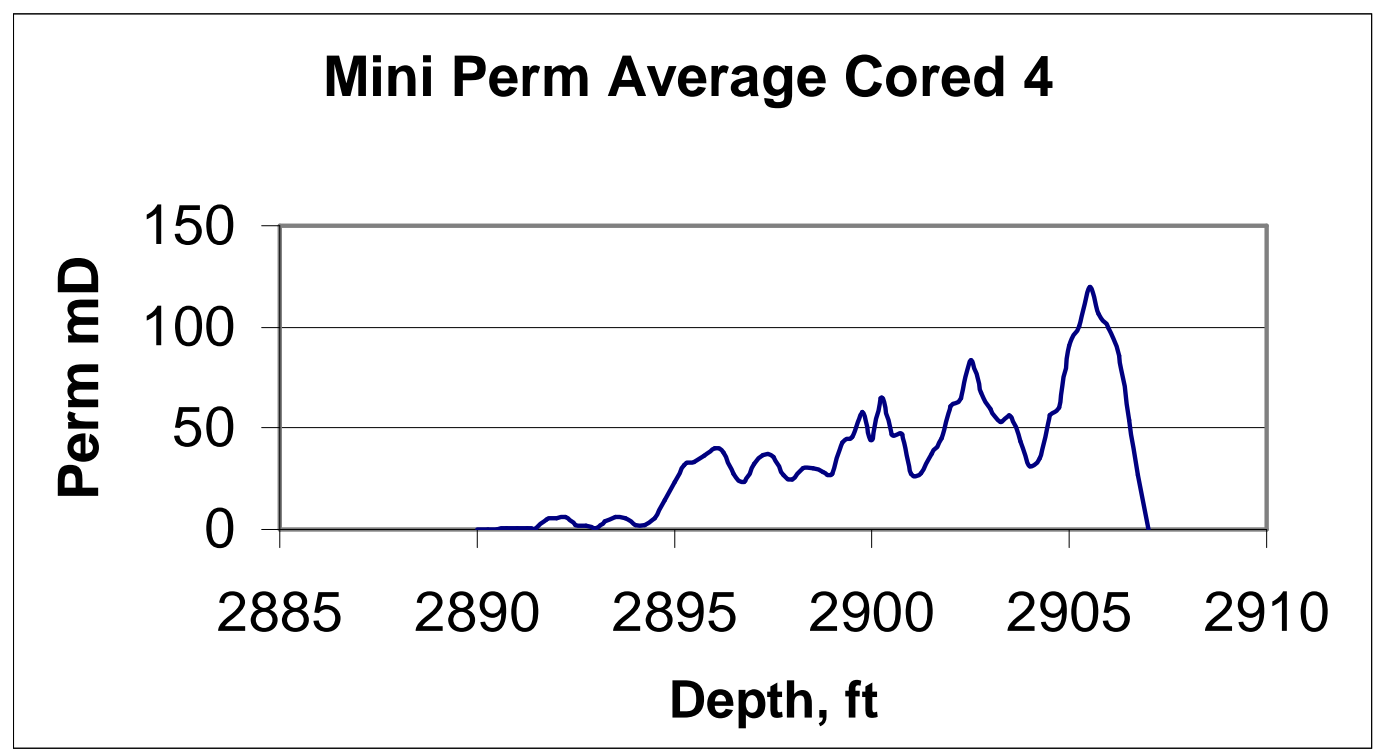

Figure A18. Averaged Minipermeameter Values for Cw4 


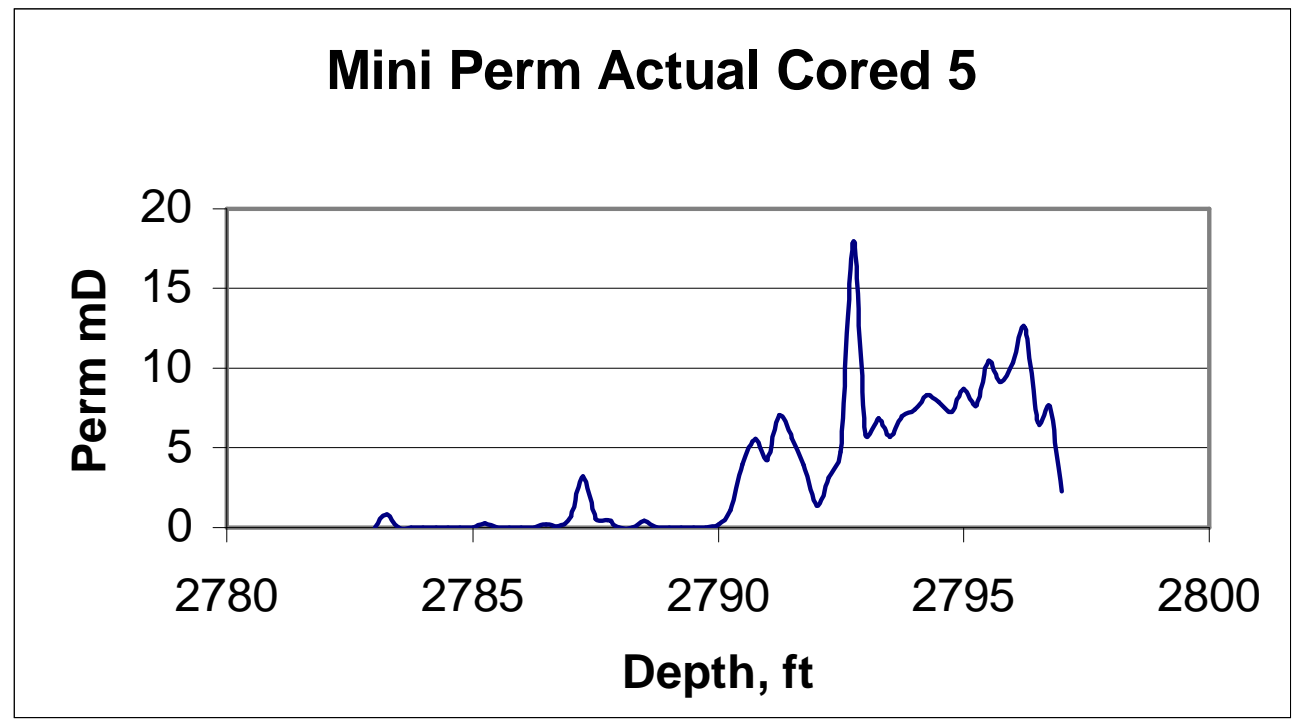

Figure A19. Actual Minipermeameter Values for Cw5

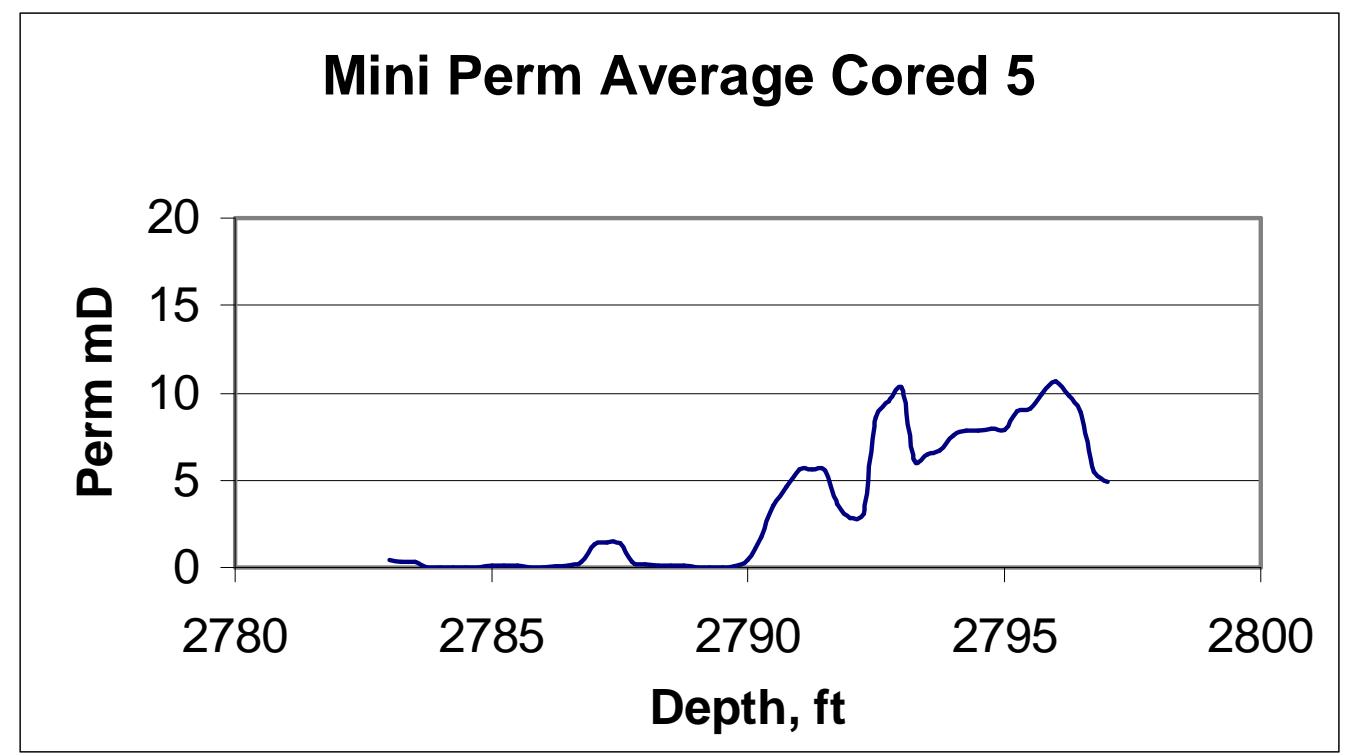

Figure A20. Averaged Minipermeameter Values for Cw5 
Appendix III - Patterns for NN One, NN Predicted and Actual

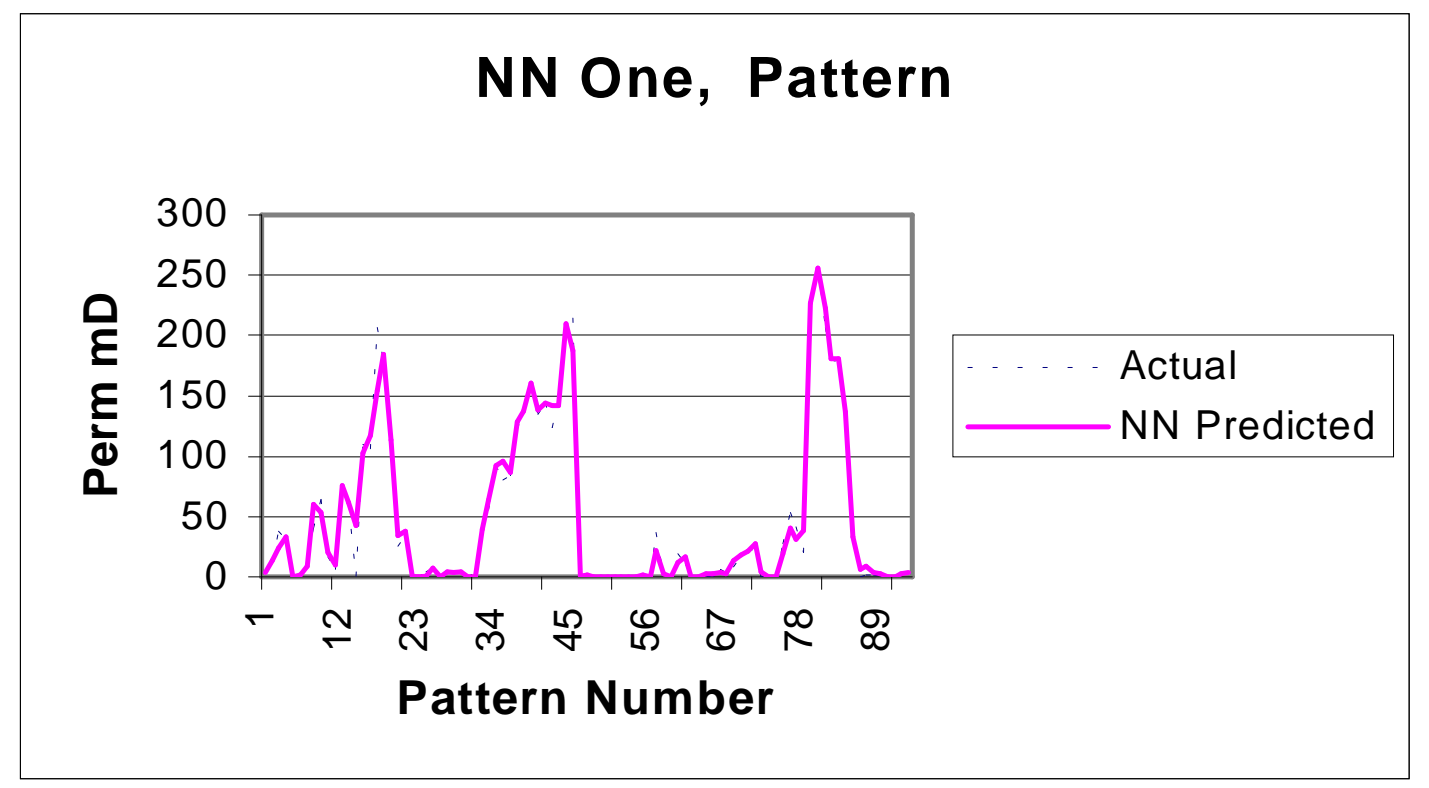

Figure A21. NN One, NN Predicted Perm. and Actual, Pattern Set

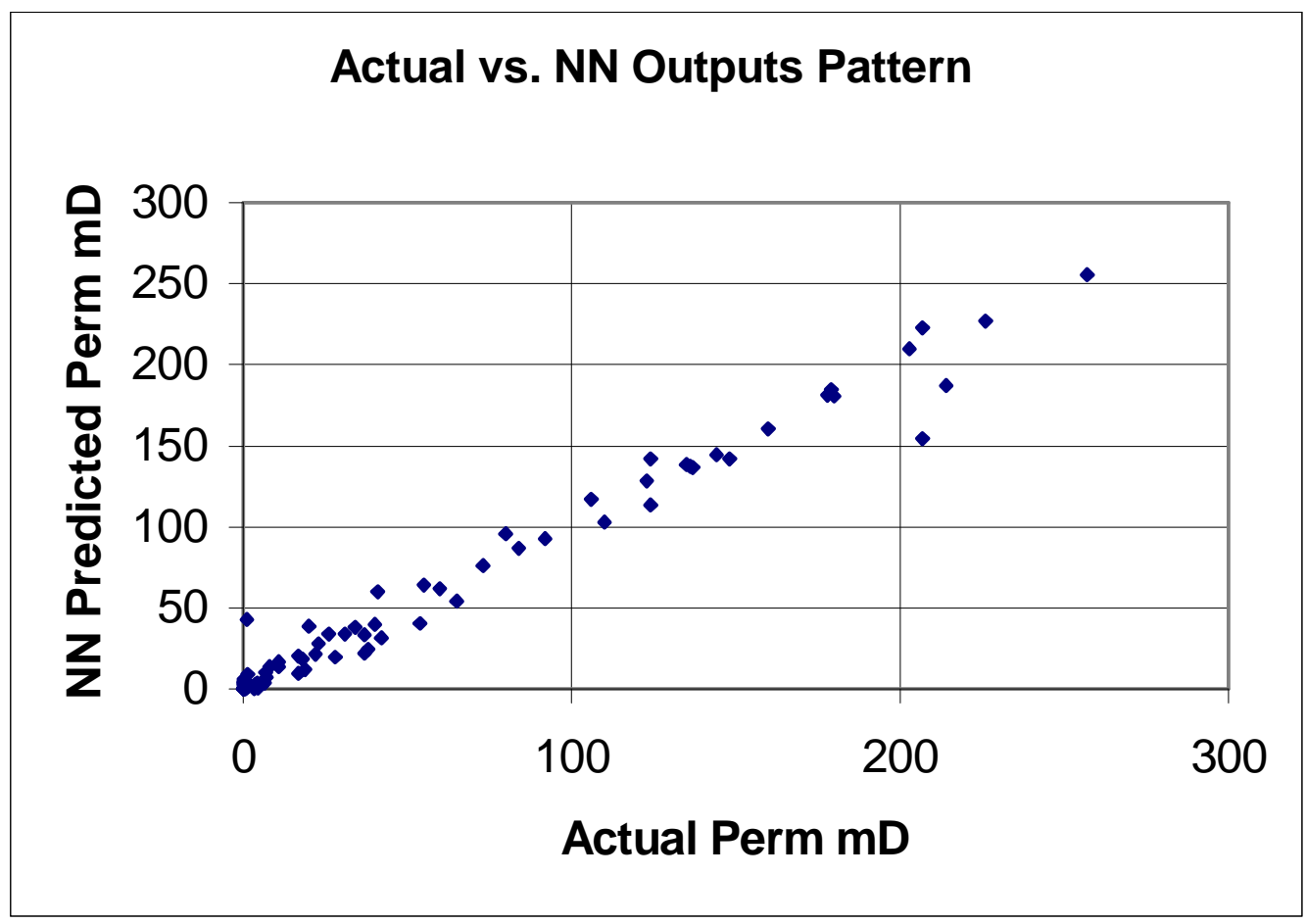

Figure A22. Actual vs. NN Predicted Perm. for NN One Applied to Pattern Set 


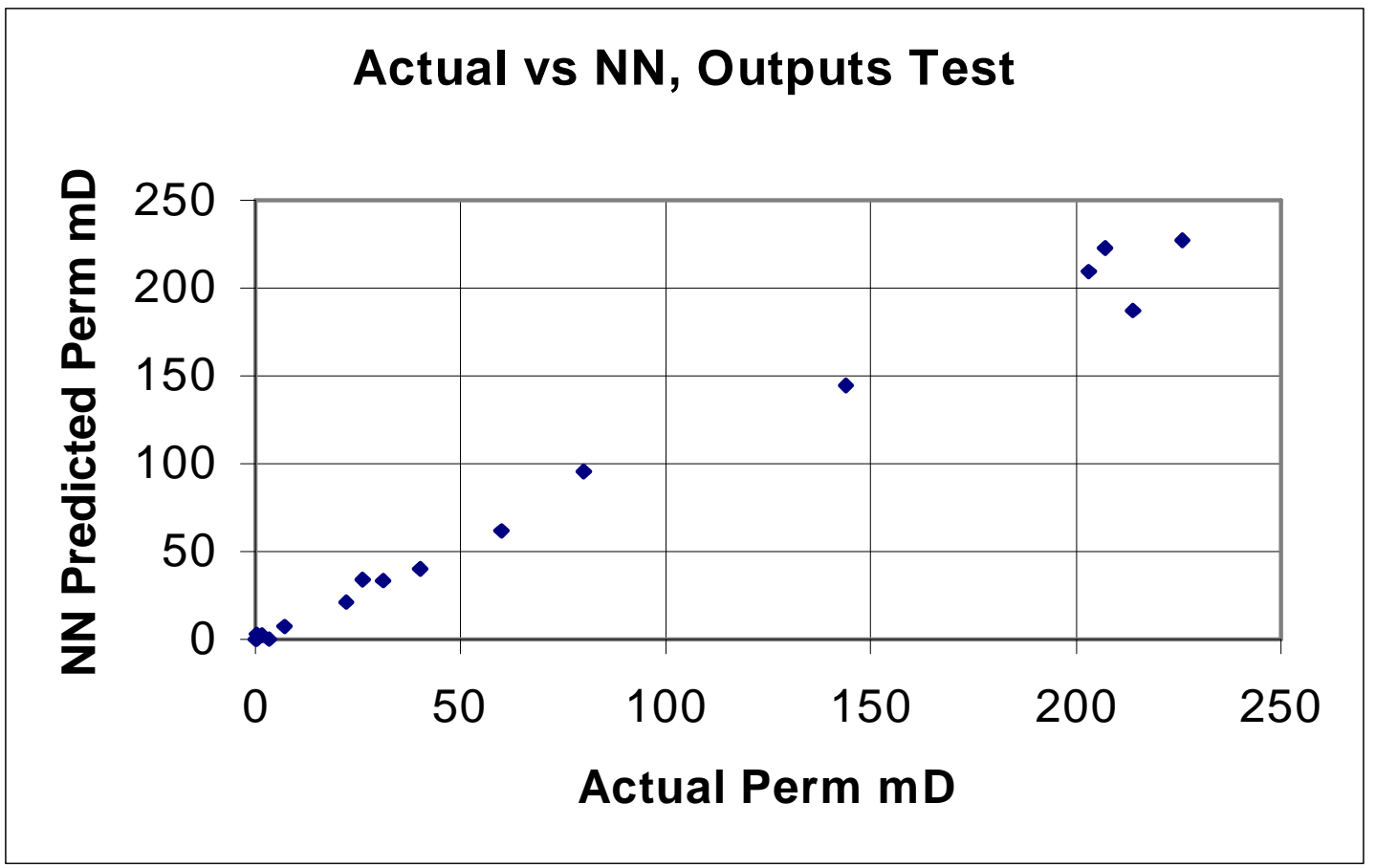

Fiaure A23. Actual vs. NN Predicted Perm. for NN One Anblied to Test Set

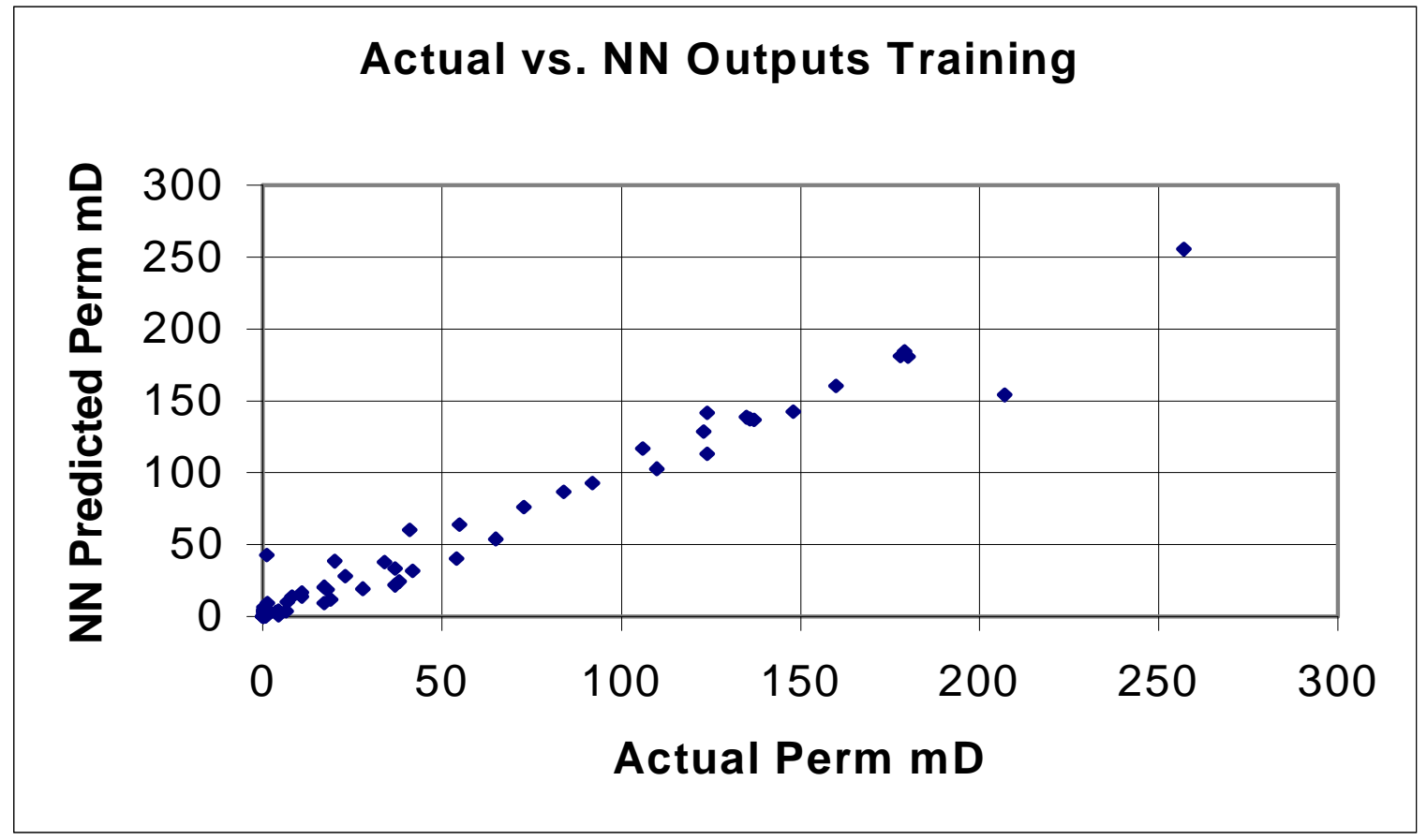

Figure A24. Actual vs. NN Predicted Perm. for NN One Applied to Training Set 


\section{Appendix IV - NN One Verification Network with Cw1 as Production Set:}

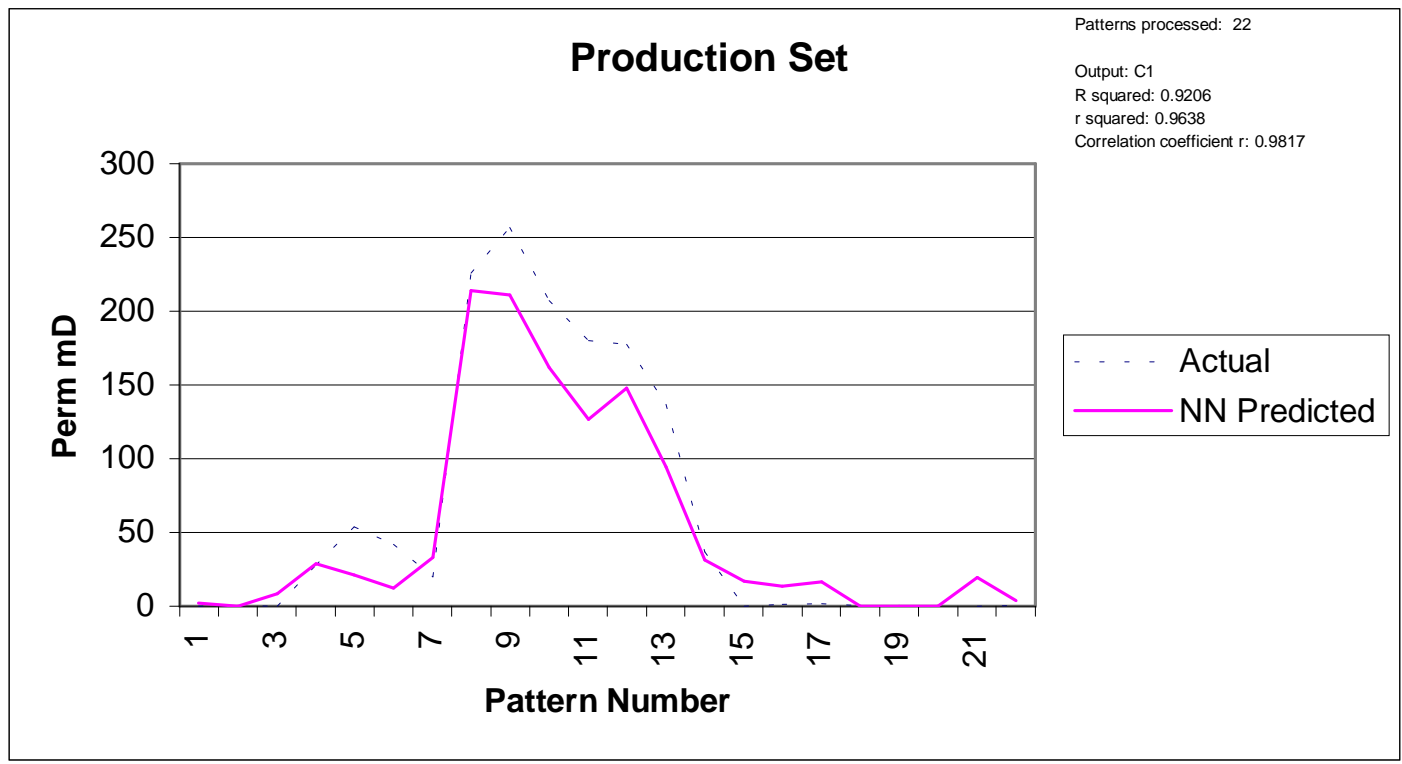

Figures A25. NN One Verification Net(Cw1), Production Set

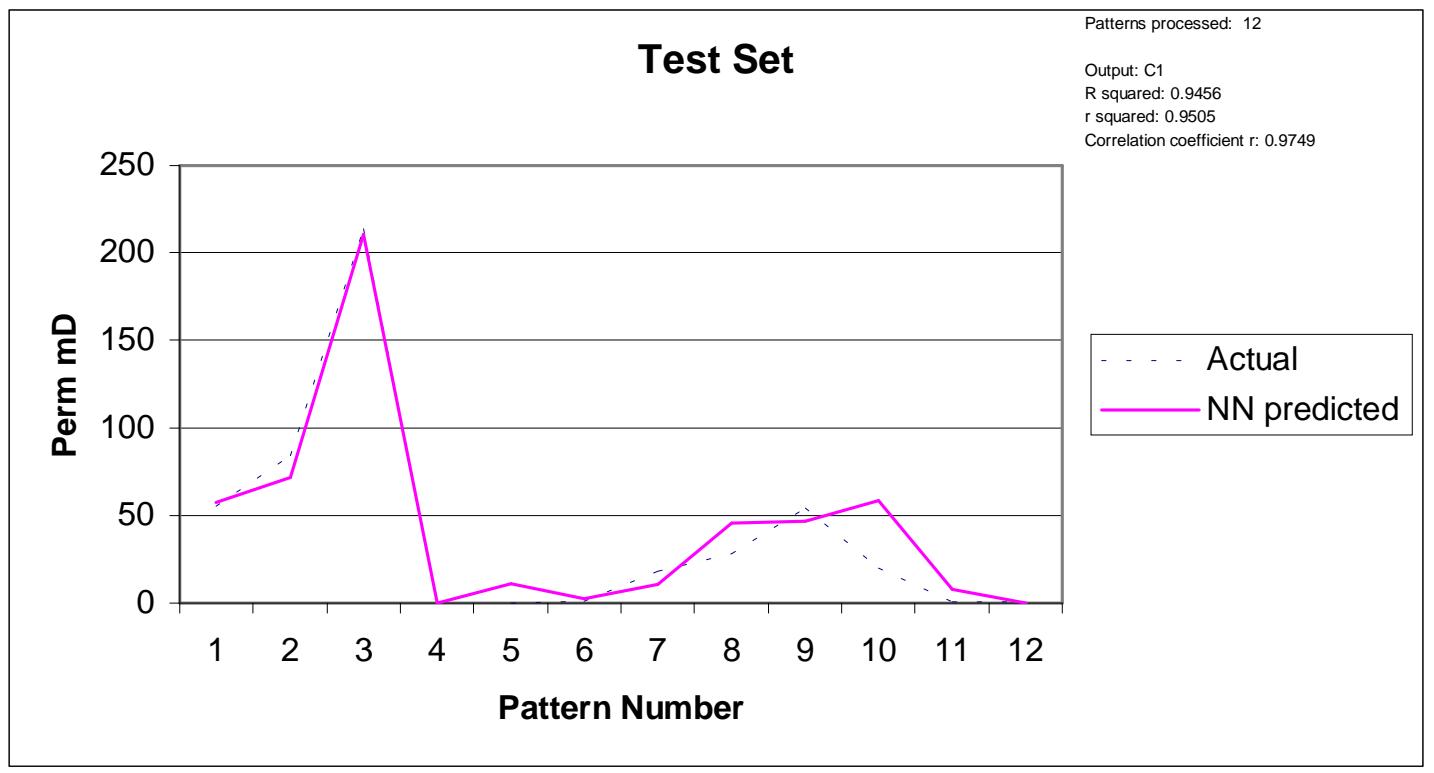

Figures A26. NN One Verification Net(Cw1), Test Set 


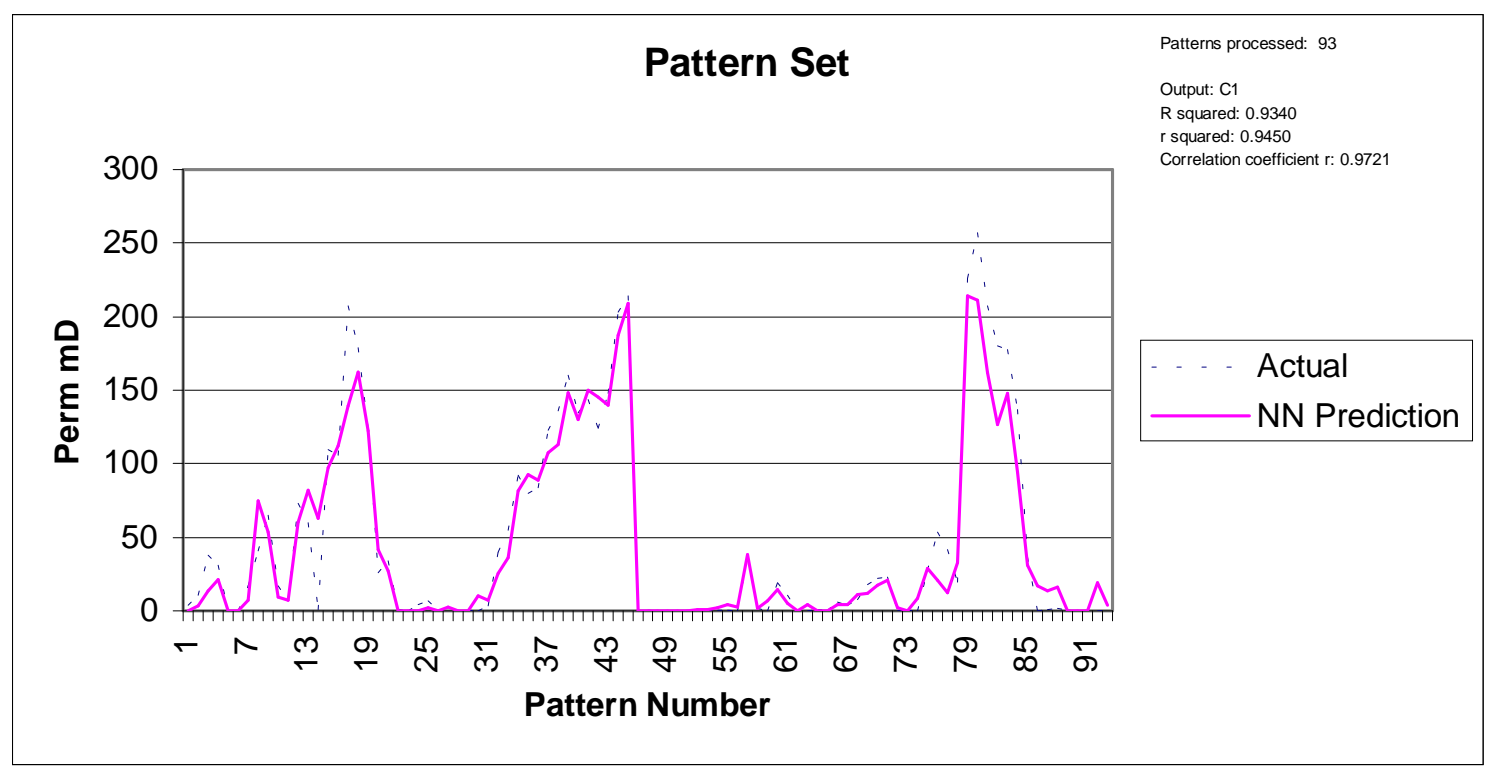

Figures A27. NN One Verification Net(Cw1), Pattern Set 


\section{Appendix V - NN One Verification Network with Cw2 as Production Set:}

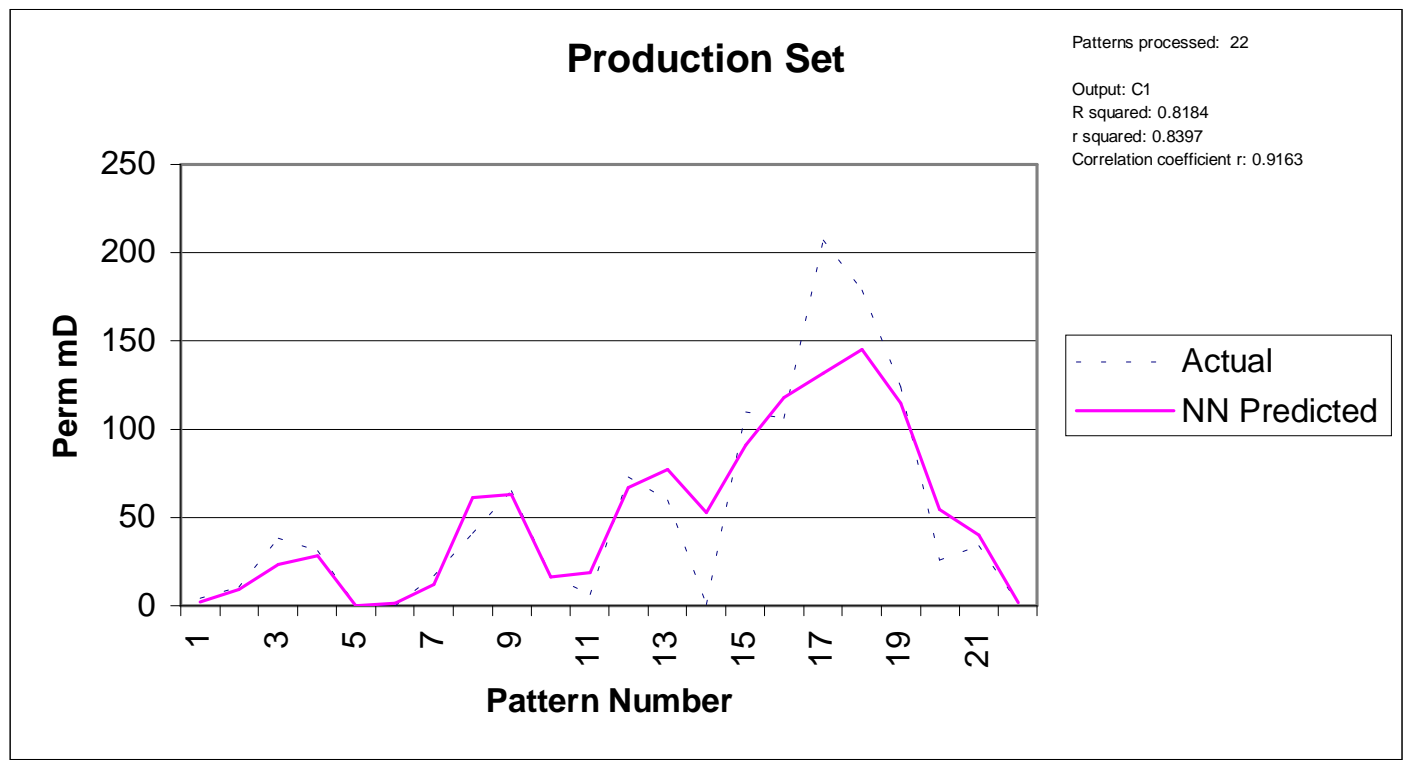

Figure A28. NN One Verification Net(Cw2), Production Set

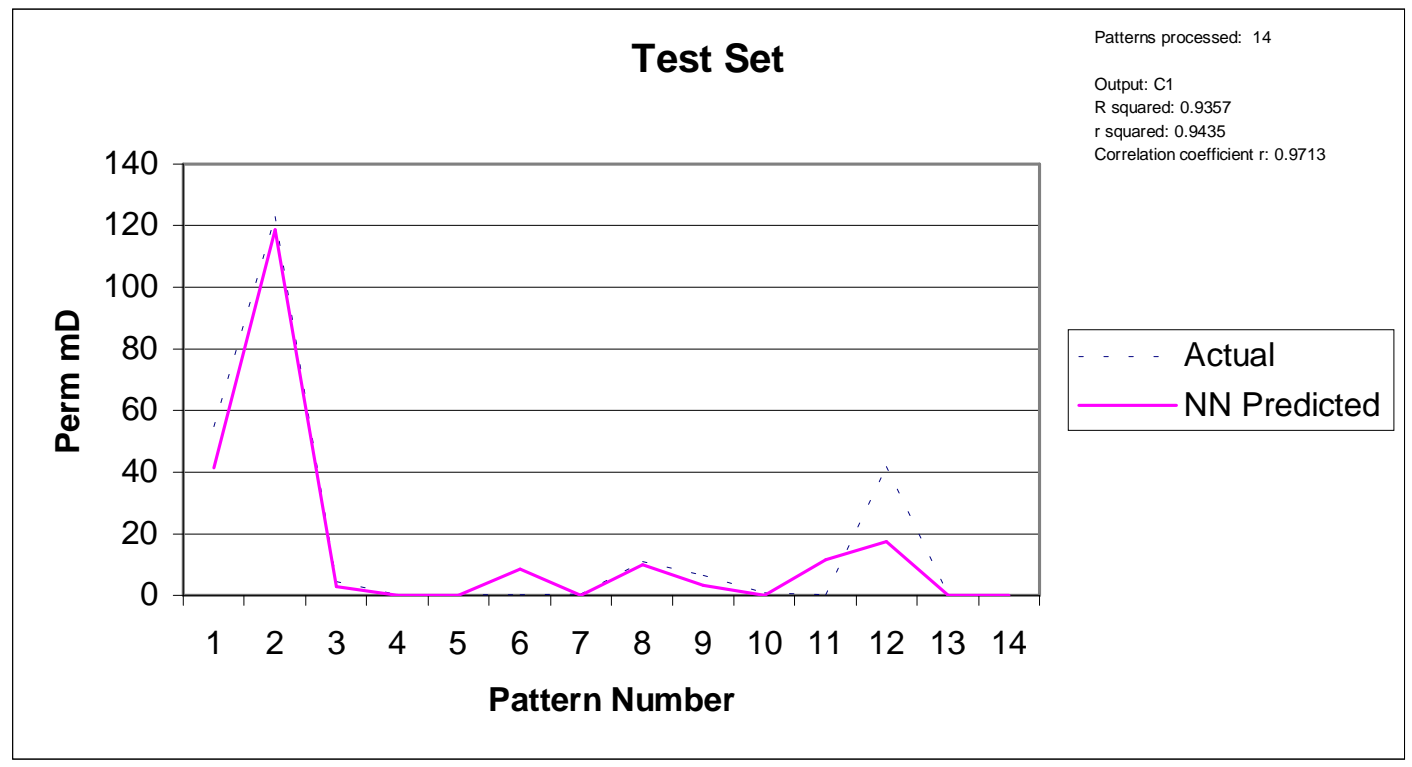

Figure A29. NN One Verification Net(Cw2), Test Set 


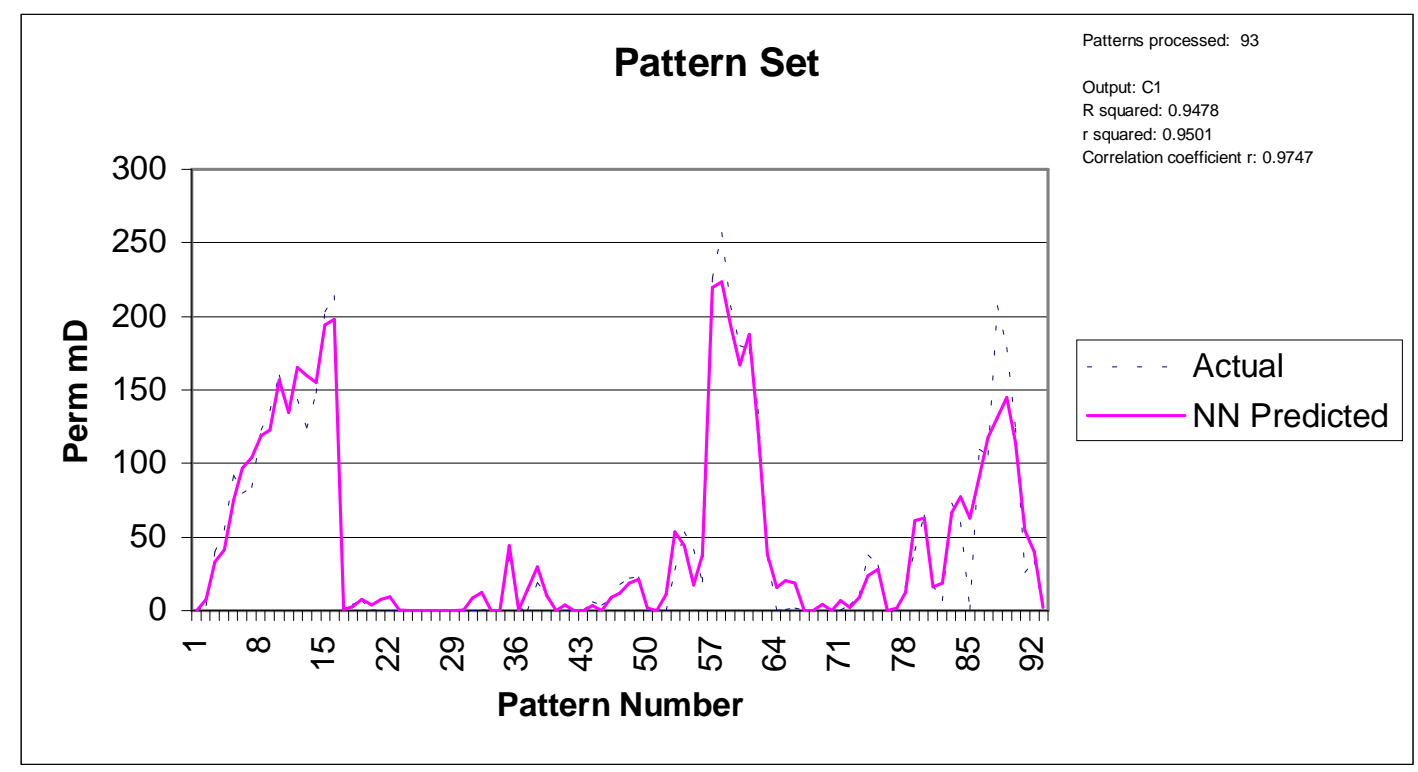

Figure A30. NN One Verification Net(Cw2), Pattern Set 


\section{Appendix VI - NN One Verification Network with Cw3 as Production Set:}

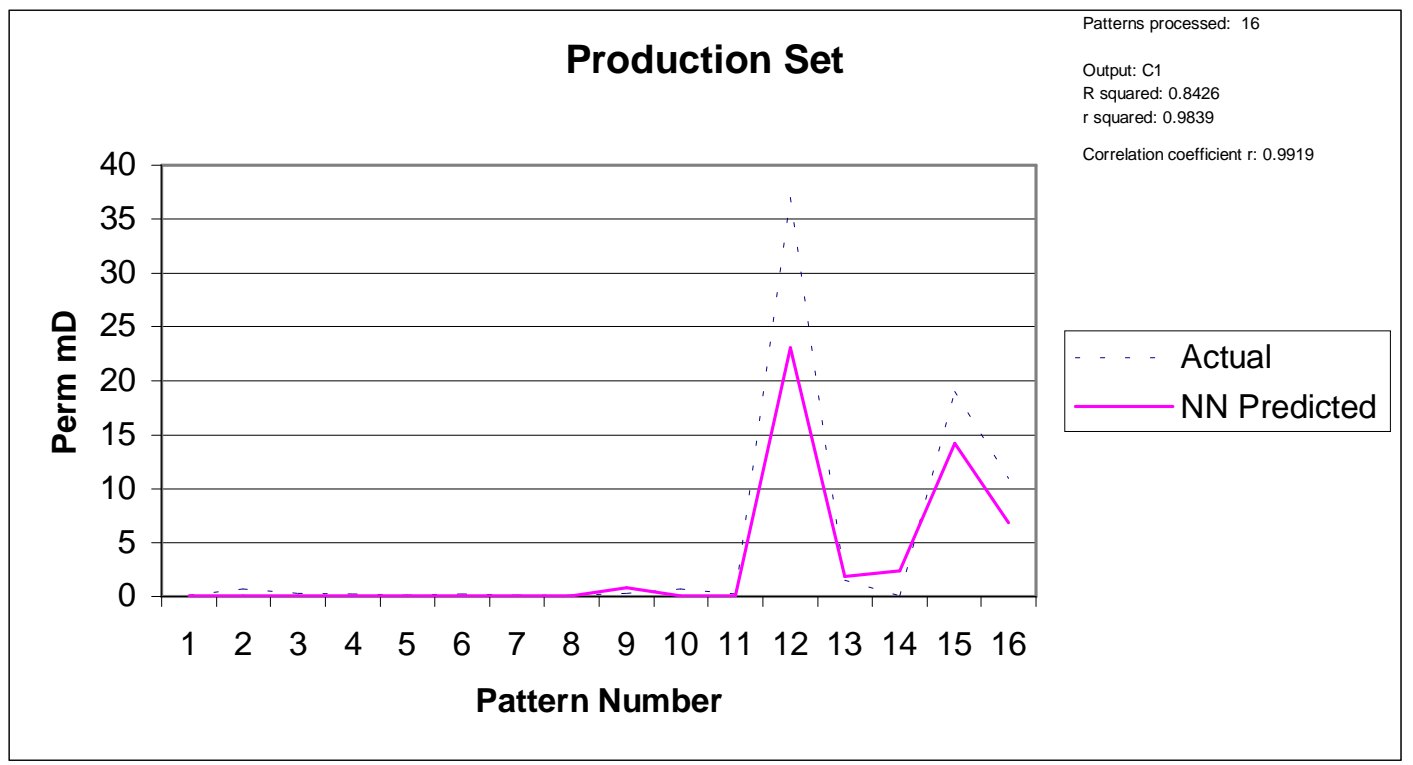

Figure A31. NN One Verification Net(Cw3), Production Set

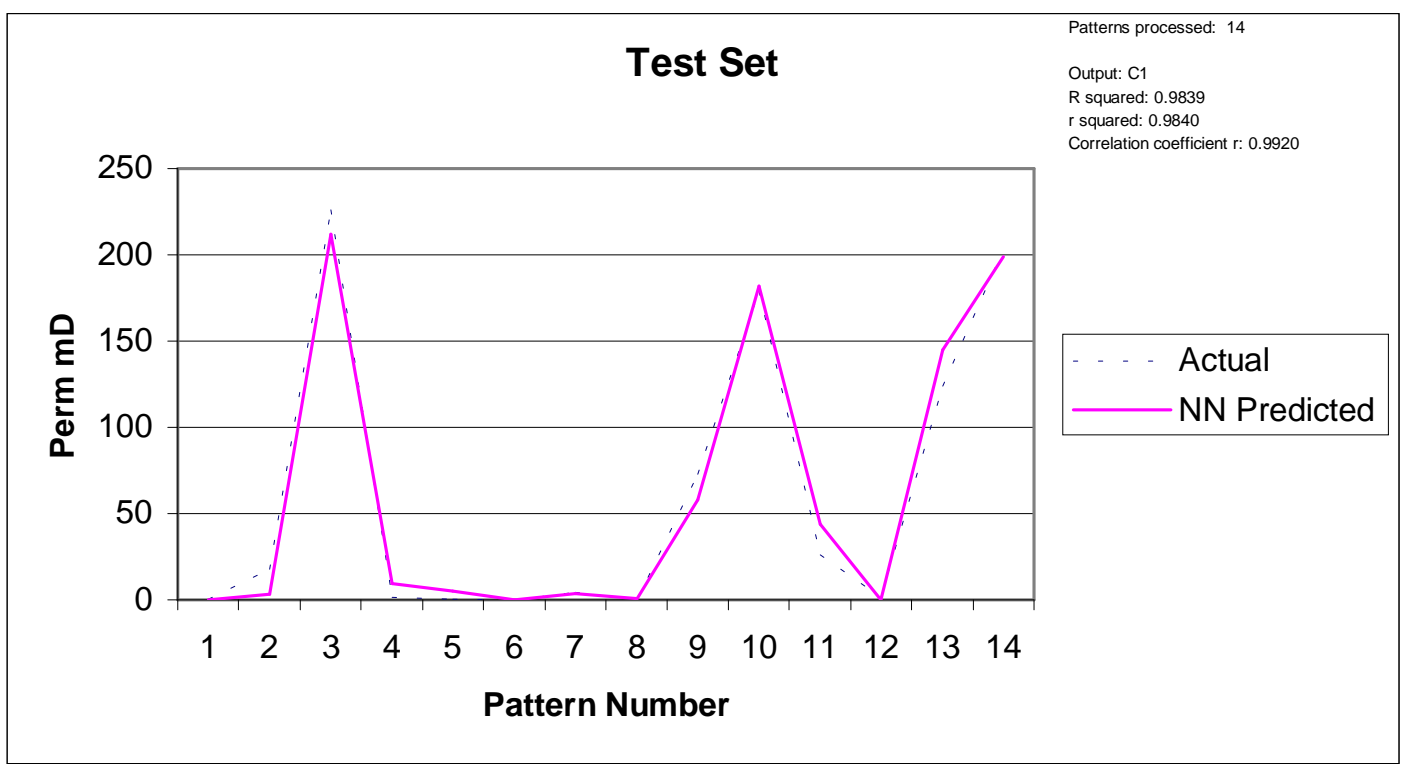

Figure A32. NN One Verification Net(Cw3), Test Set 


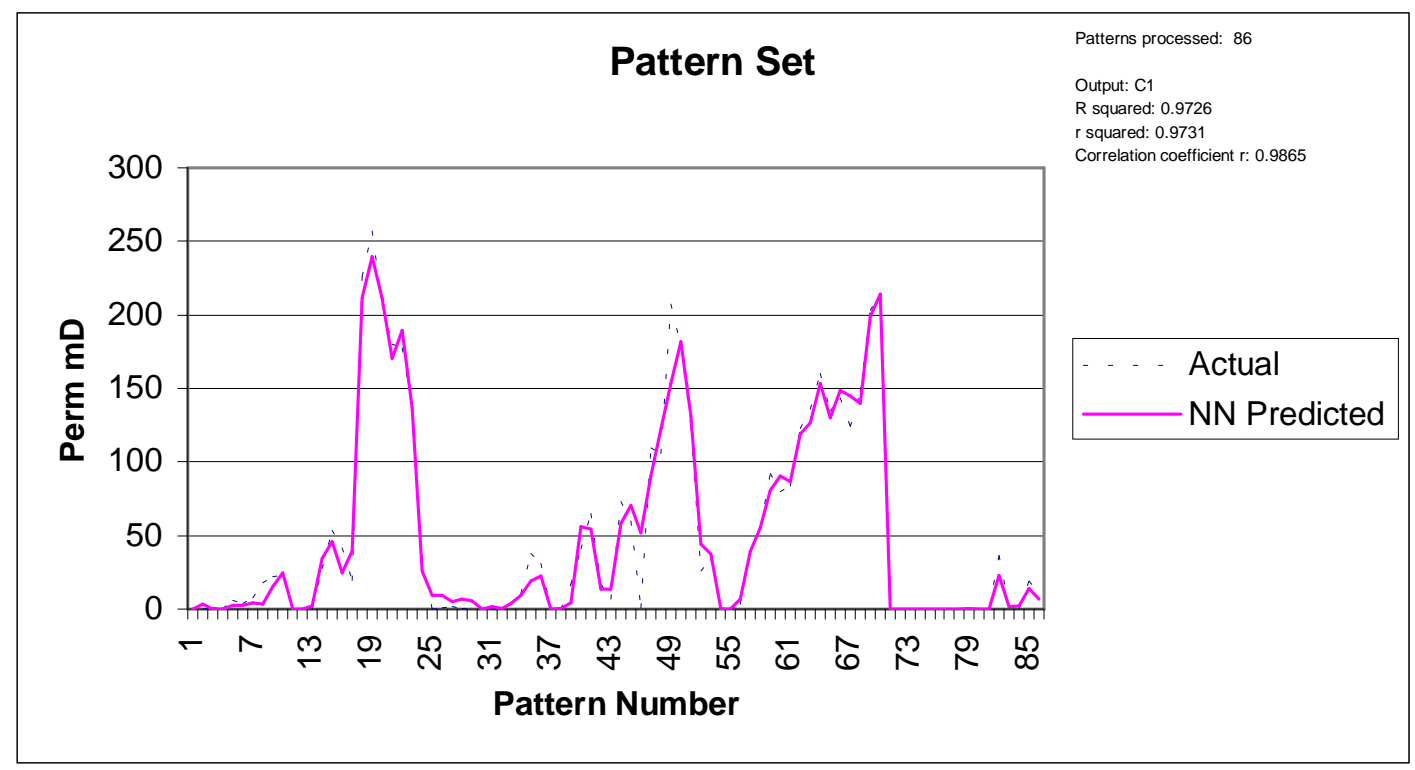

Figure A33. NN One Verification Net(Cw3), Pattern Set 
Appendix VII - NN One Verification Network with Cw4 as Production Set:

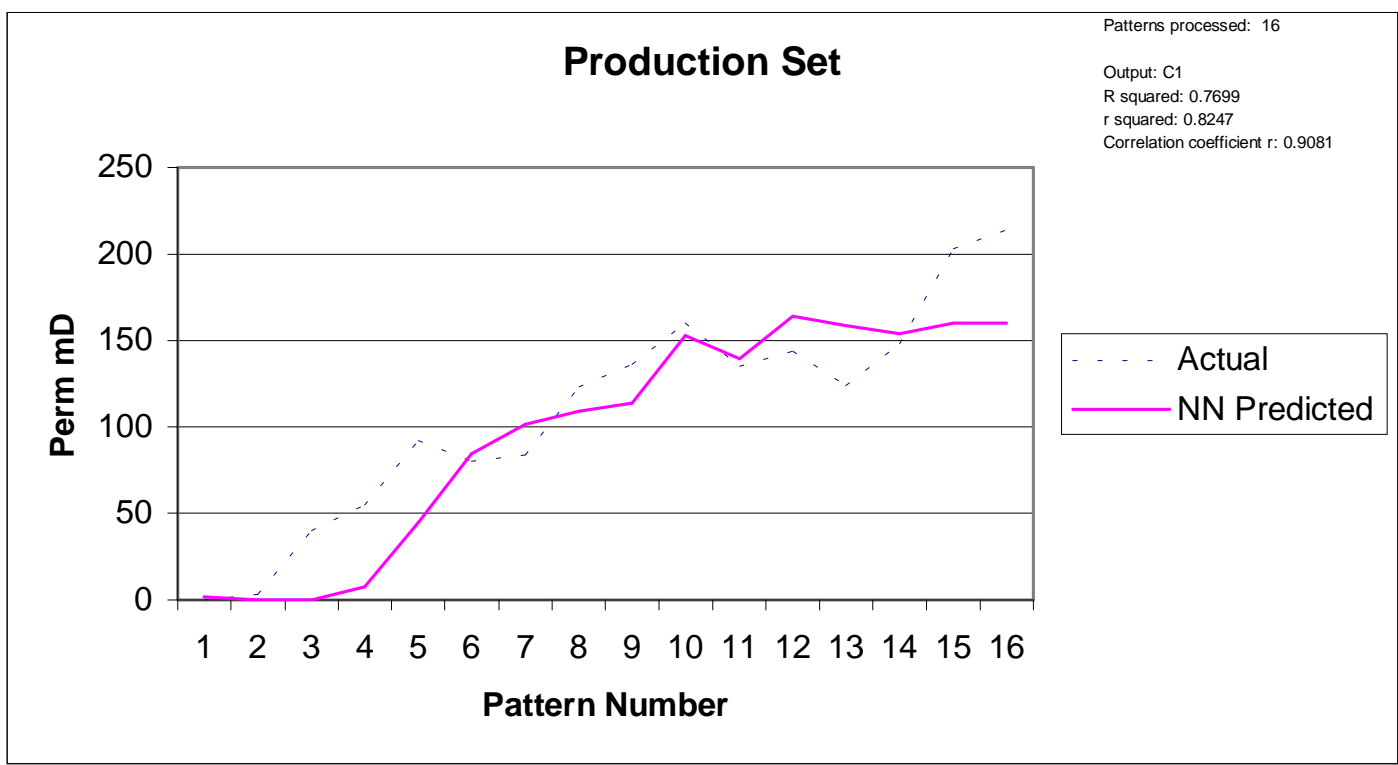

Figure A34. NN One Verification Net(Cw4), Production Set

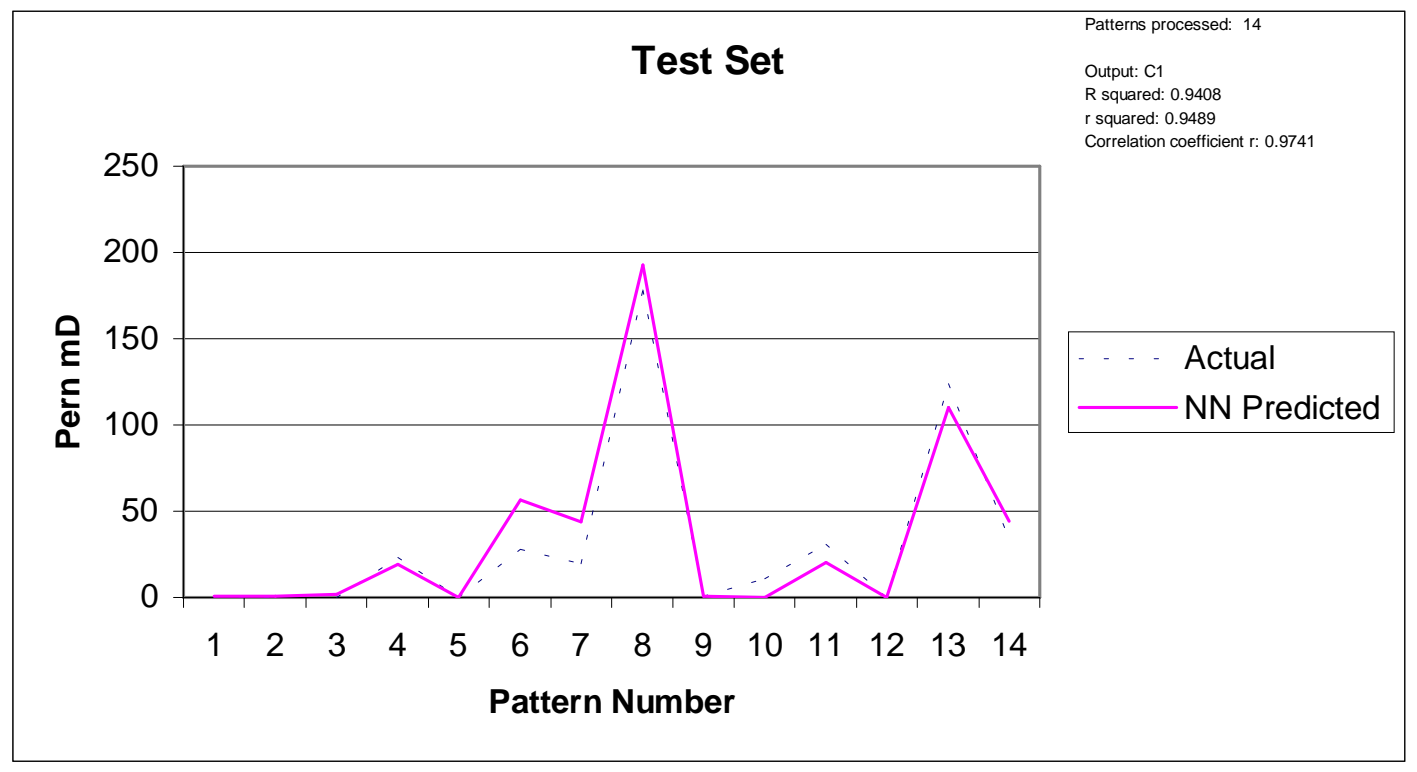

Figure A35. NN One Verification Net(Cw4), Test Set 


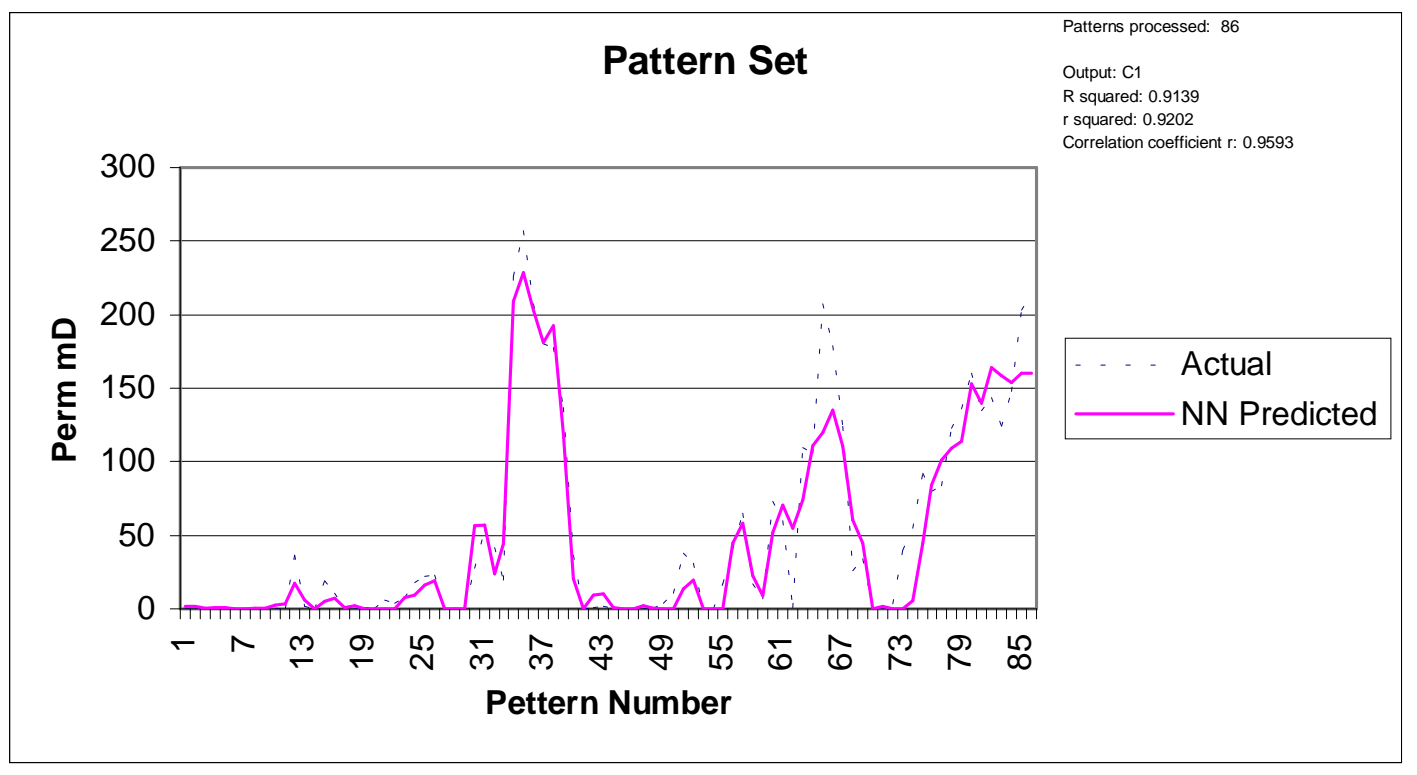

Figure A36. NN One Verification Net(Cw4), Pattern Set 


\section{Appendix VIII - NN One Verification Network with Cw5 as Production Set:}

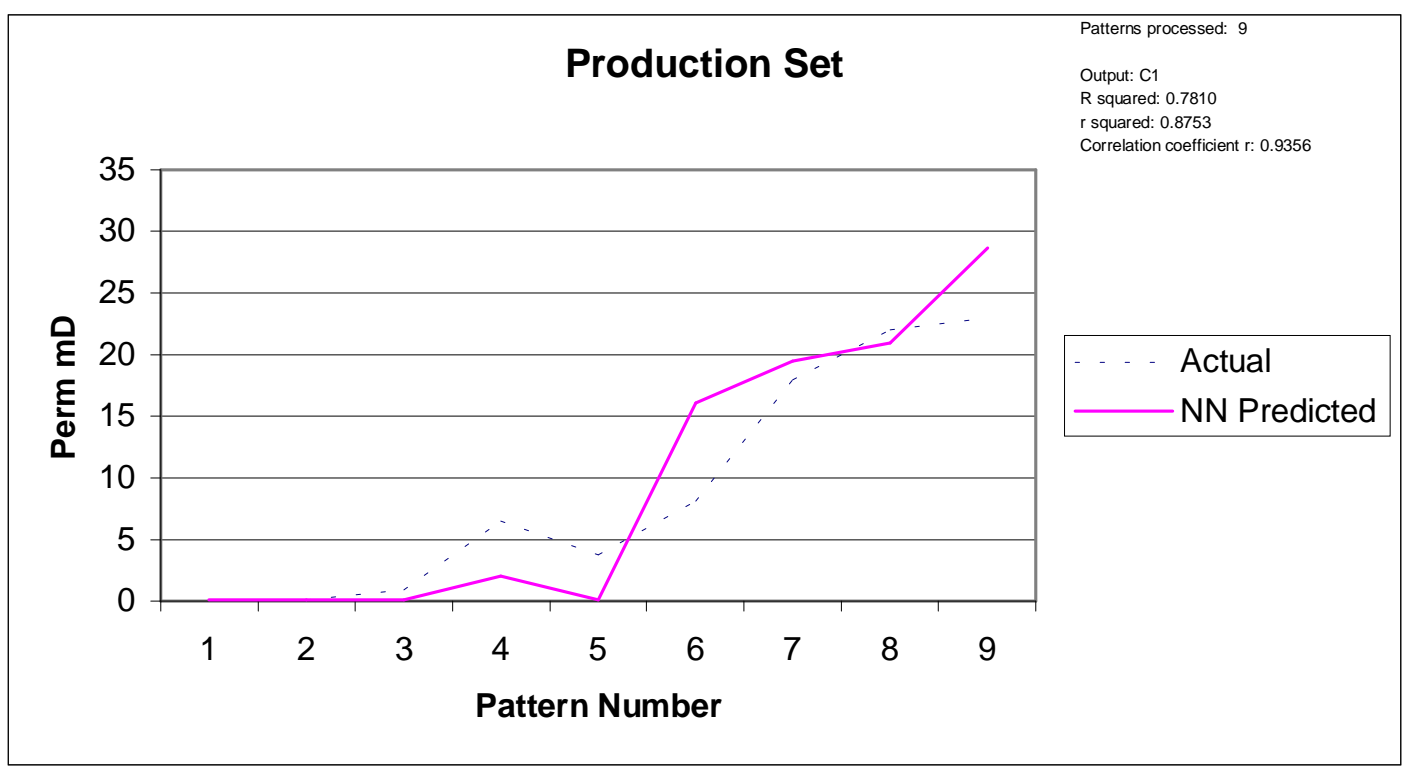

Figure A37. NN One Verification Net(Cw5), Production Set

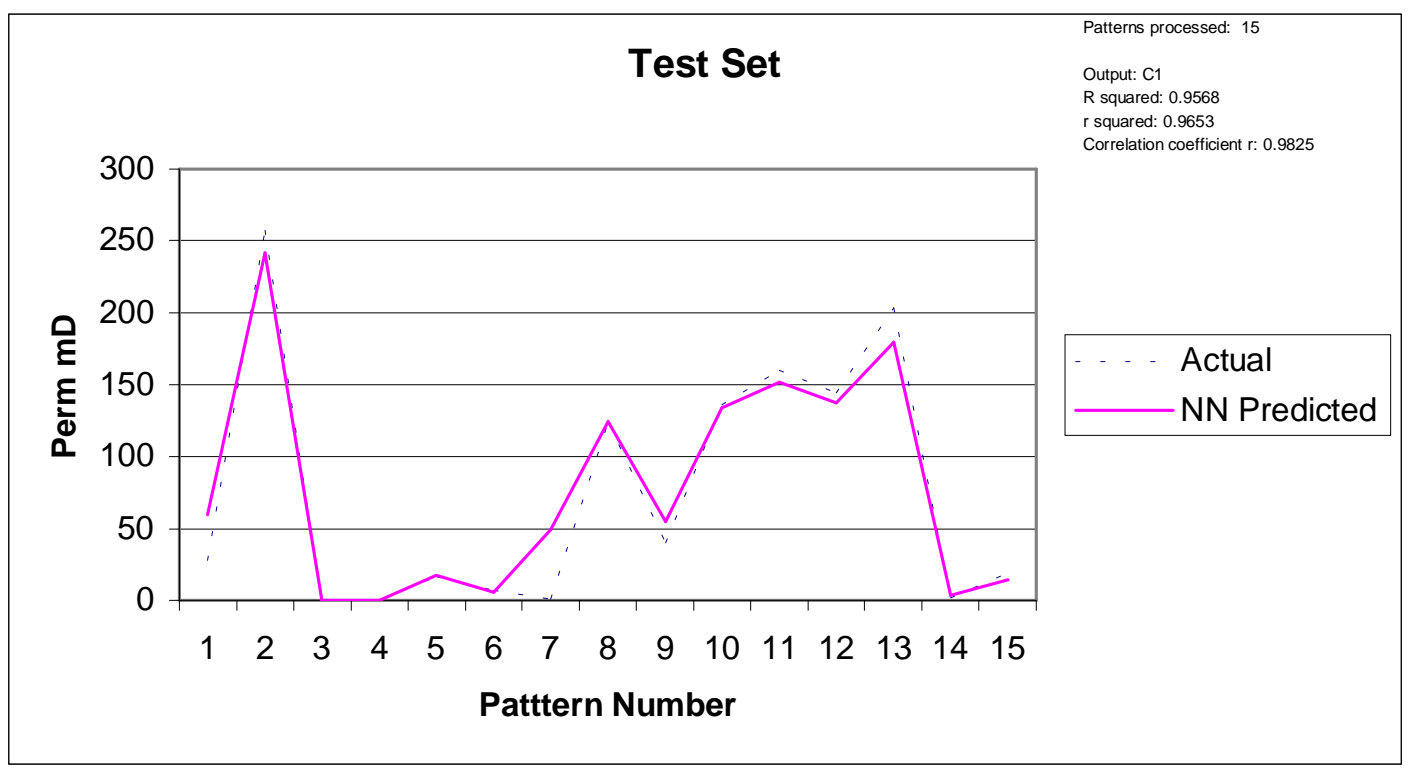

Figure A38. NN One Verification Net(Cw5), Test Set 


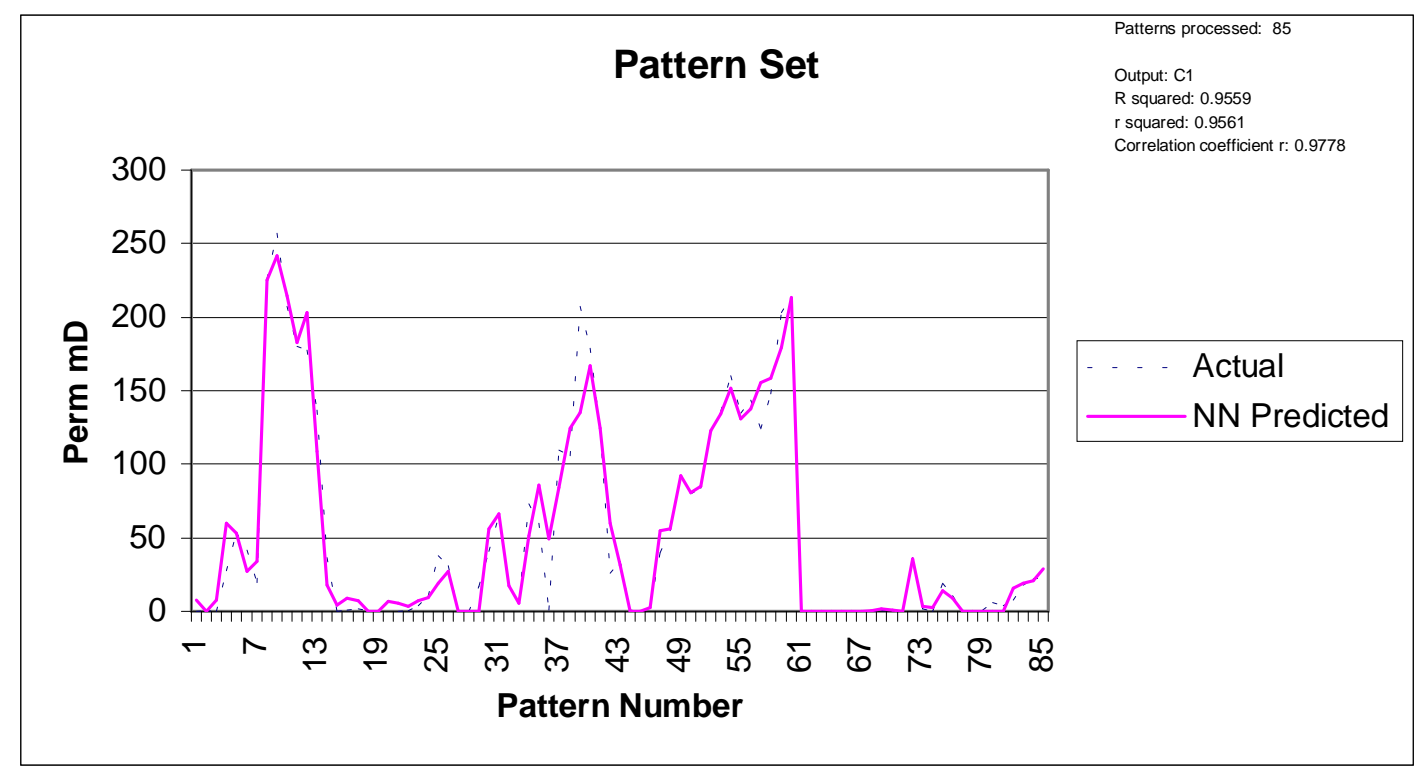

Figure A39. NN One Verification Net(Cw5), Pattern Set 
Appendix IX - Patterns for NN Two, NN Predicted and Actual

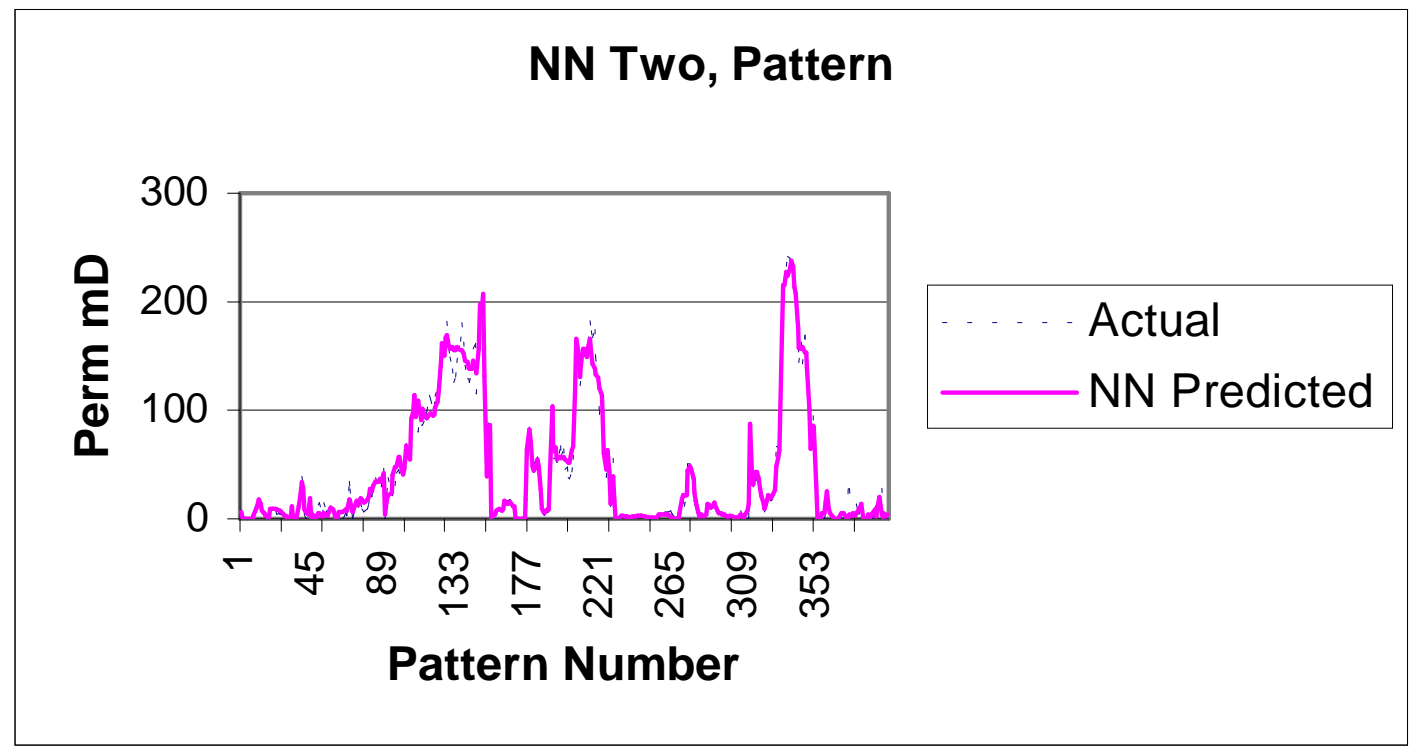

Figure A40. NN Two, NN Predicted and Actual Perm., Pattern Set

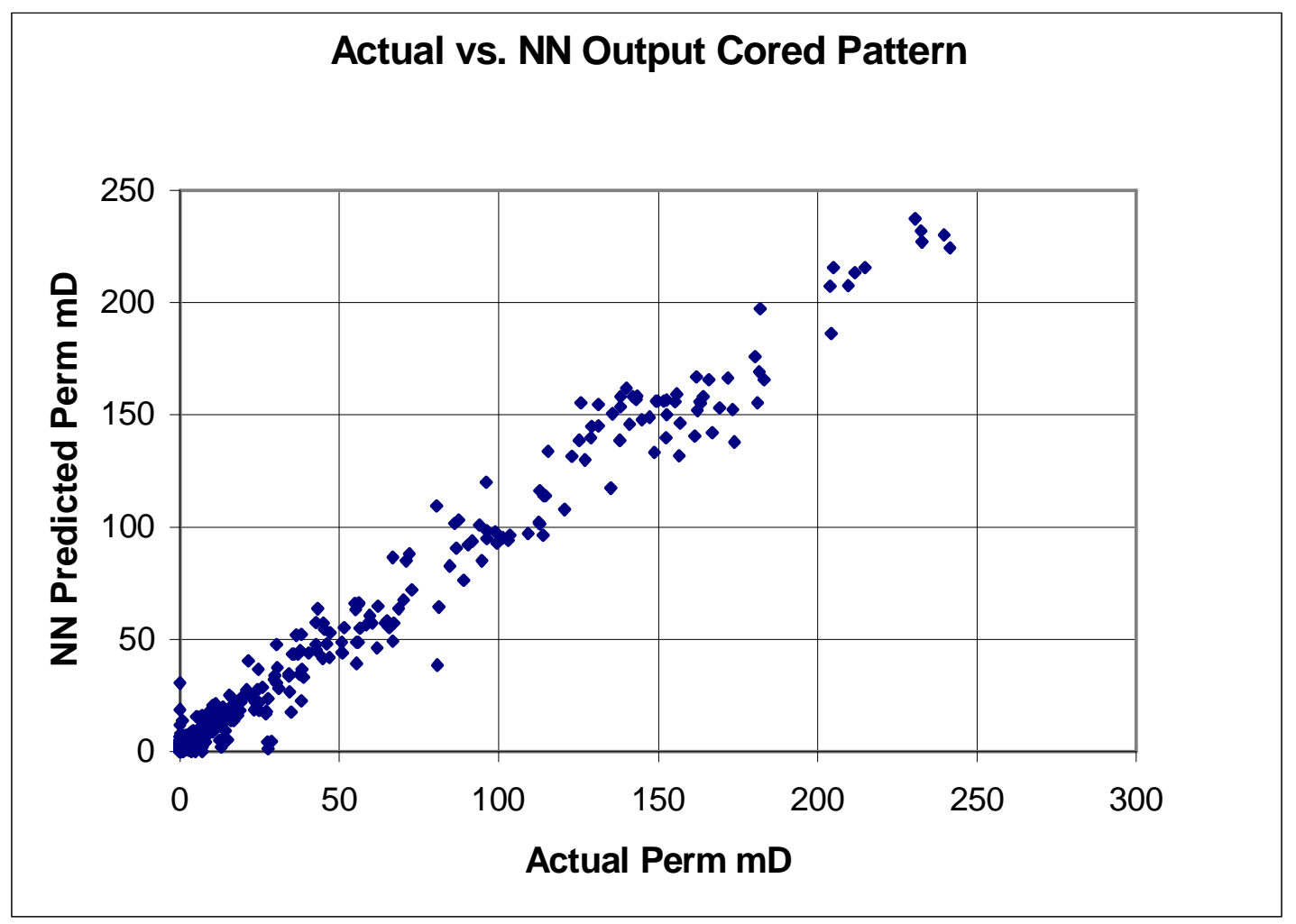

Figure A41. Actual vs. NN Predicted Perm. for NN Two Applied to Pattern Set 


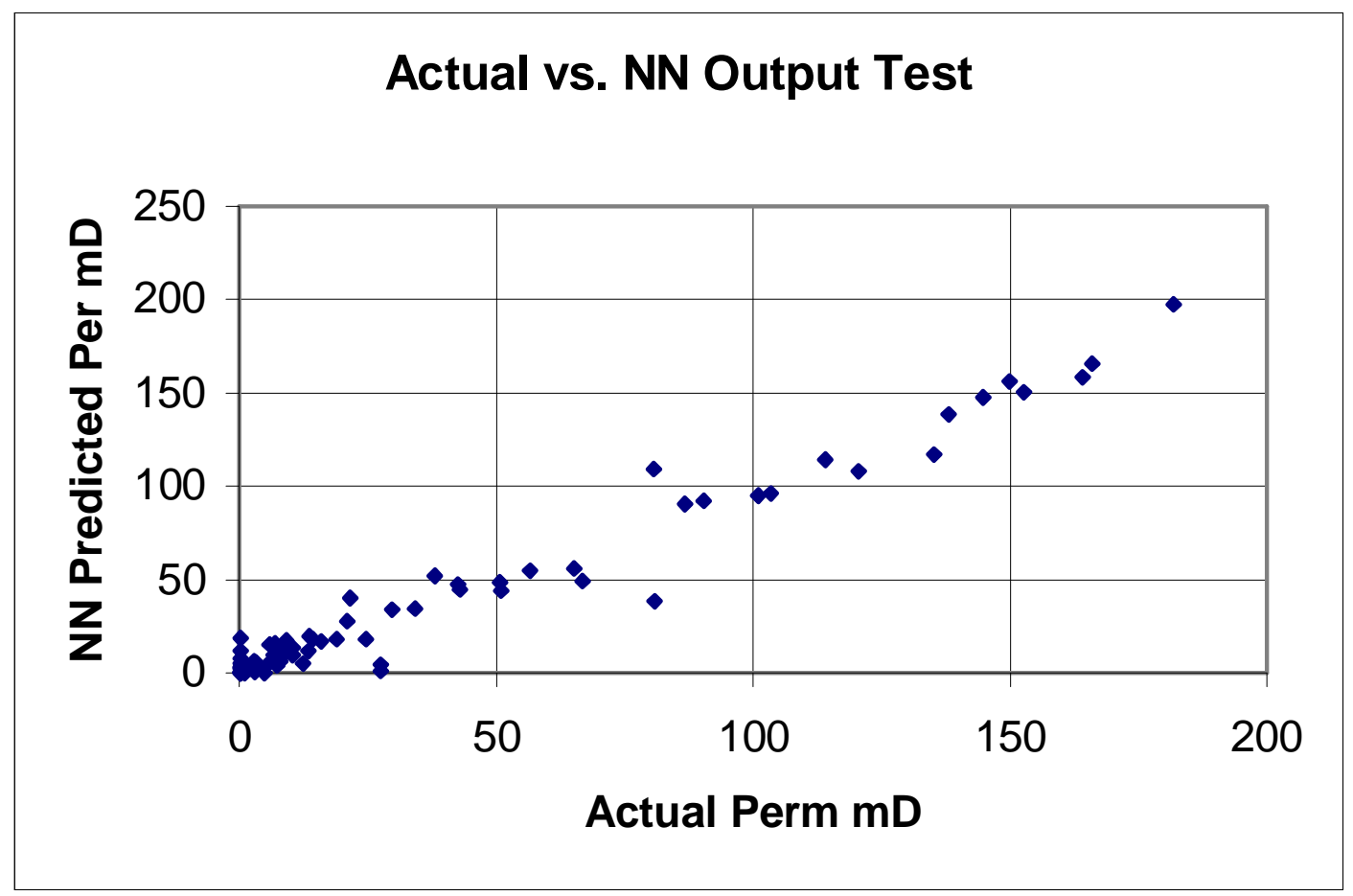

Figure A42. Actual vs. NN Predicted for NN Two Applied to Test Set

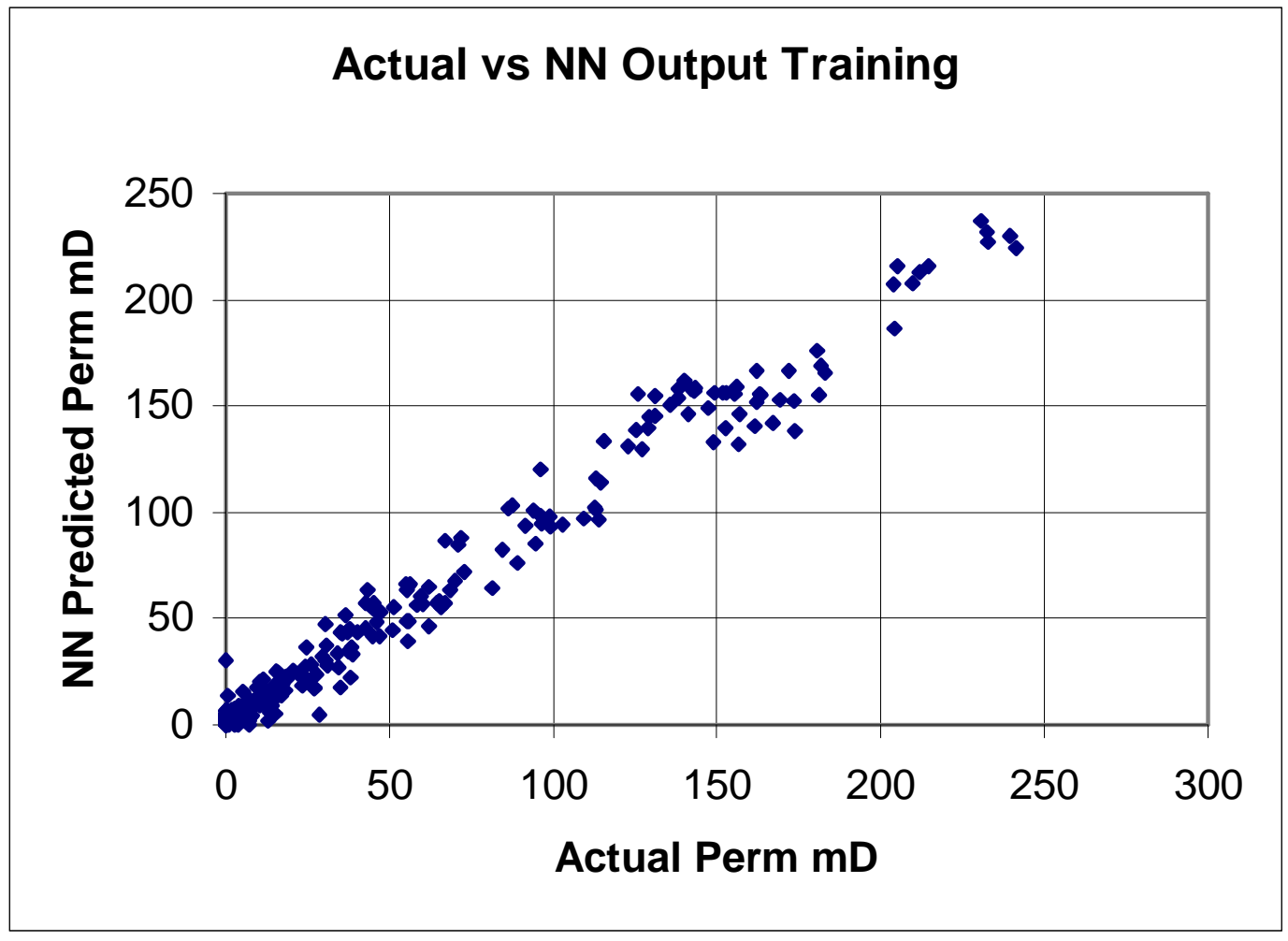

Fiaure A43. Actual vs. NN Predicted Perm. for NN Two Apblied to Trainina Set 


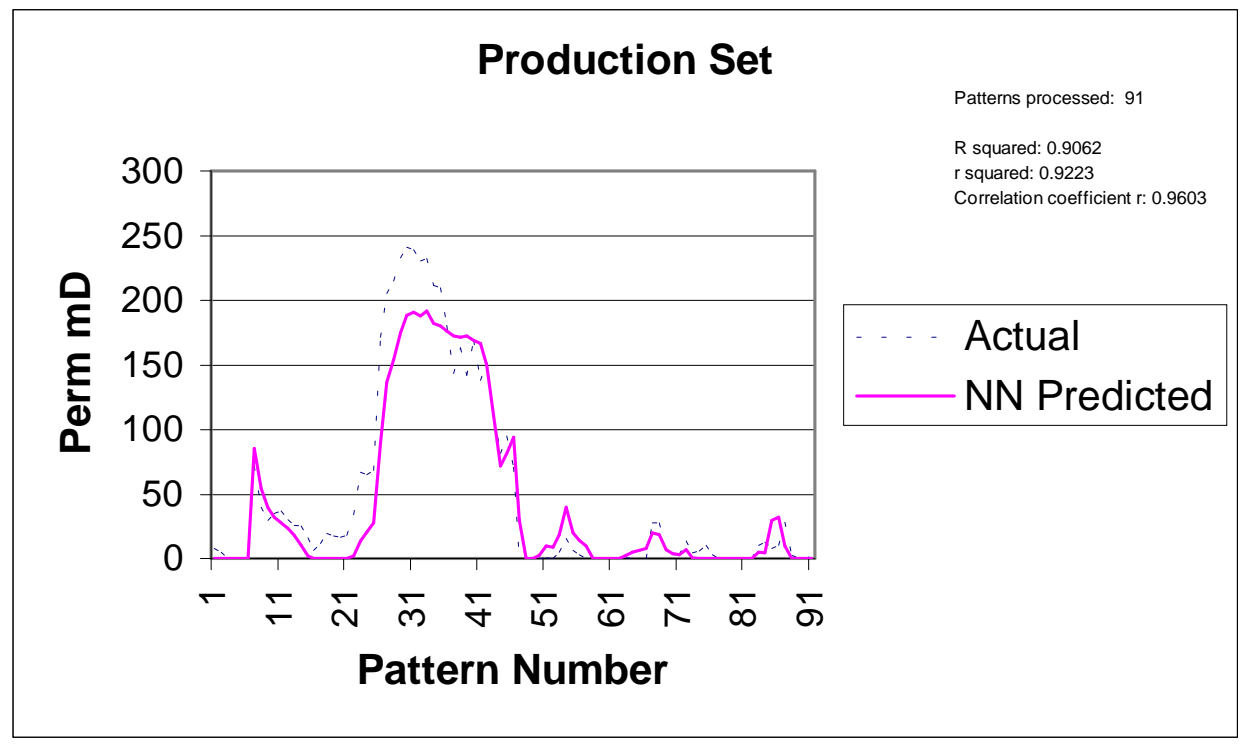

Figure A44. NN Two Verification Net(Cw1), Production Set

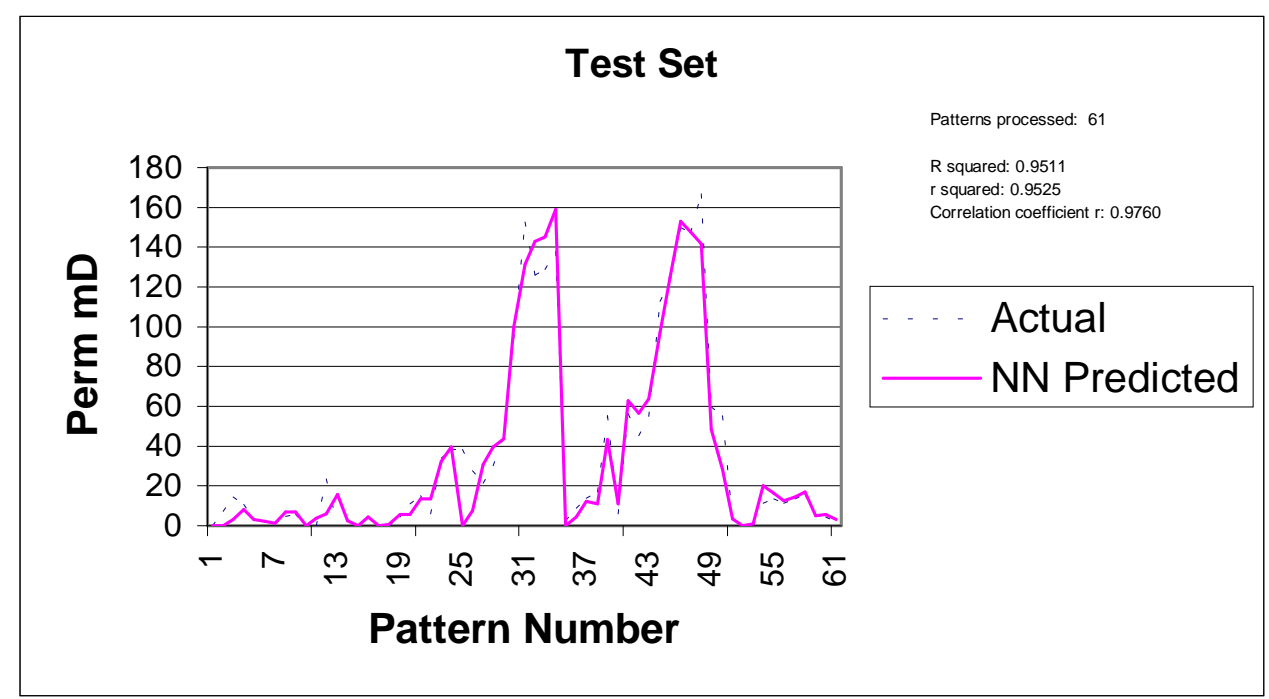

Figure A45. NN Two Verification Net(Cw1), Test Set 


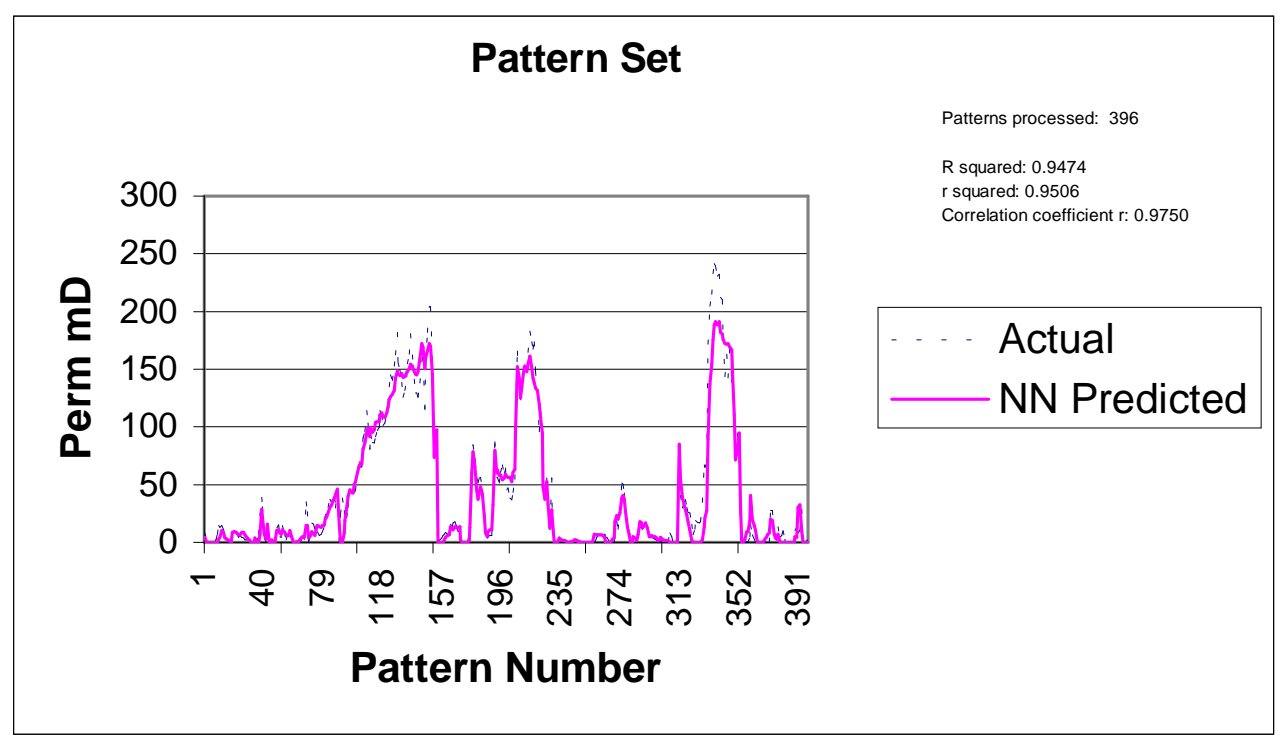

Figure A46. NN Two Verification Net(Cw1), Pattern Set 


\section{Appendix XI - NN Two Verification Network with Cw2 as Production Set:}

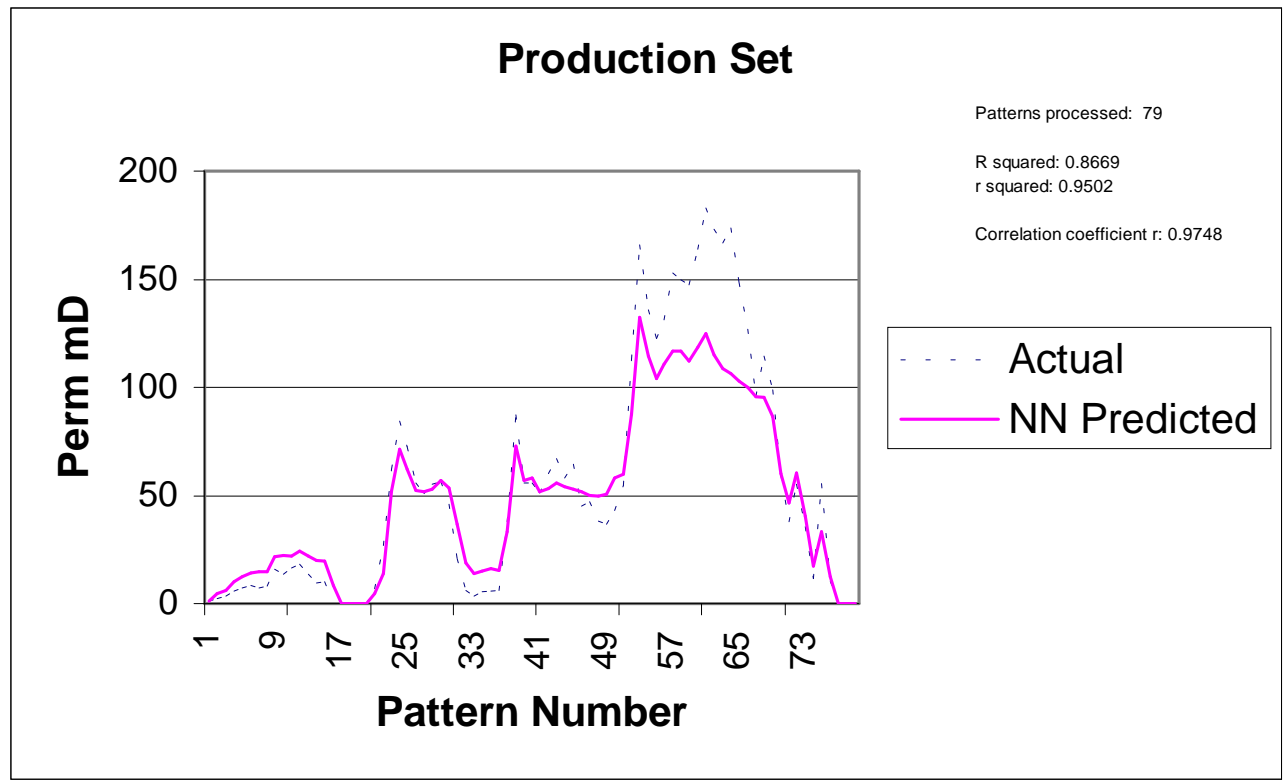

Figure A47. NN Two Verification Net(Cw2), Production Set

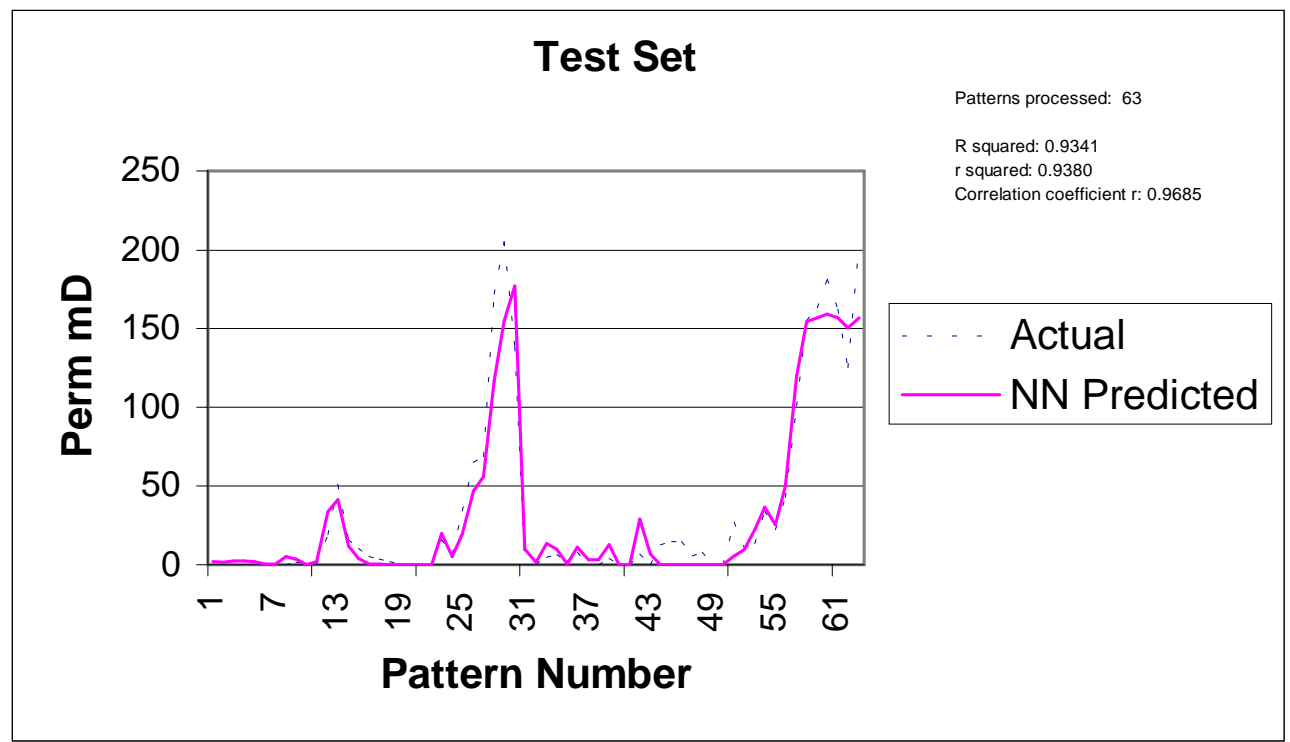

Figure A48. NN Two Verification Net(Cw2), Test Set 


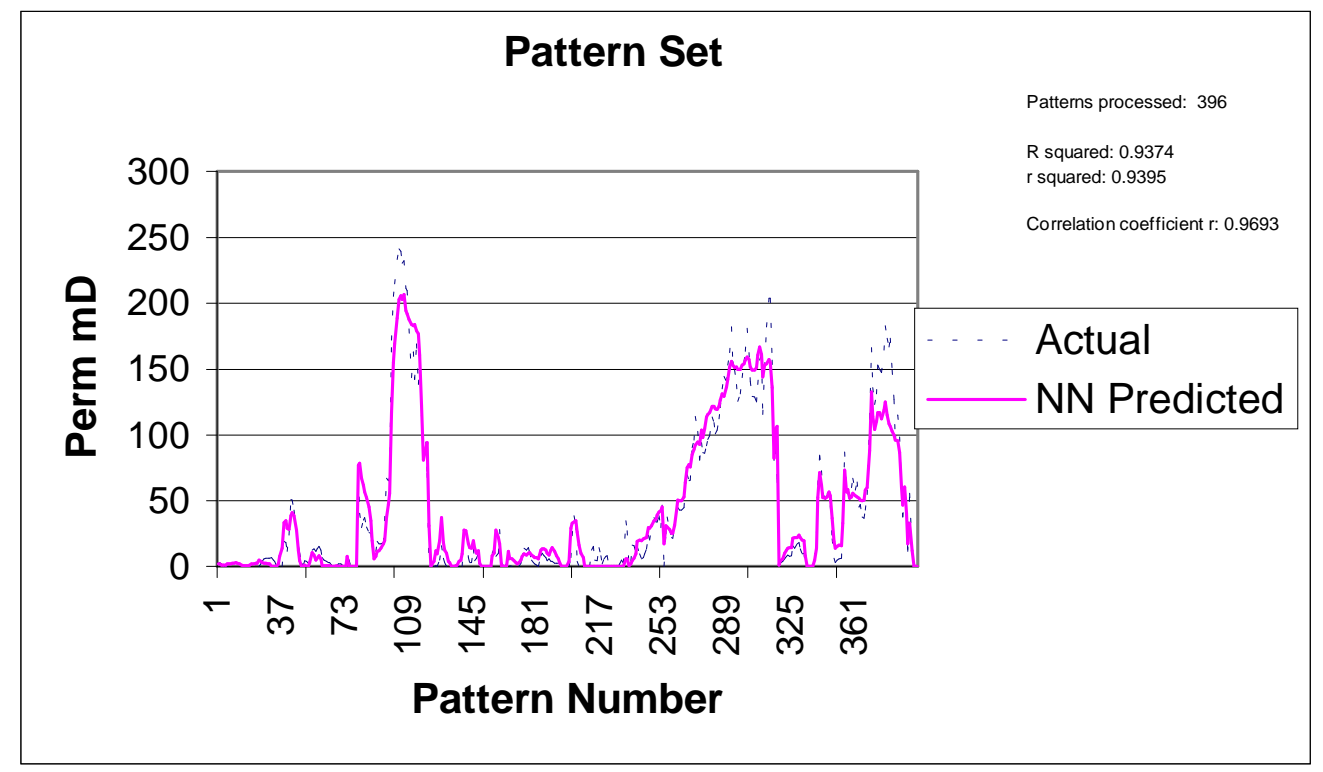

Figure A49. NN Two Verification Net(Cw2), Pattern Set 
Appendix XII - NN Two Verification Network with Cw3 as Production Set:

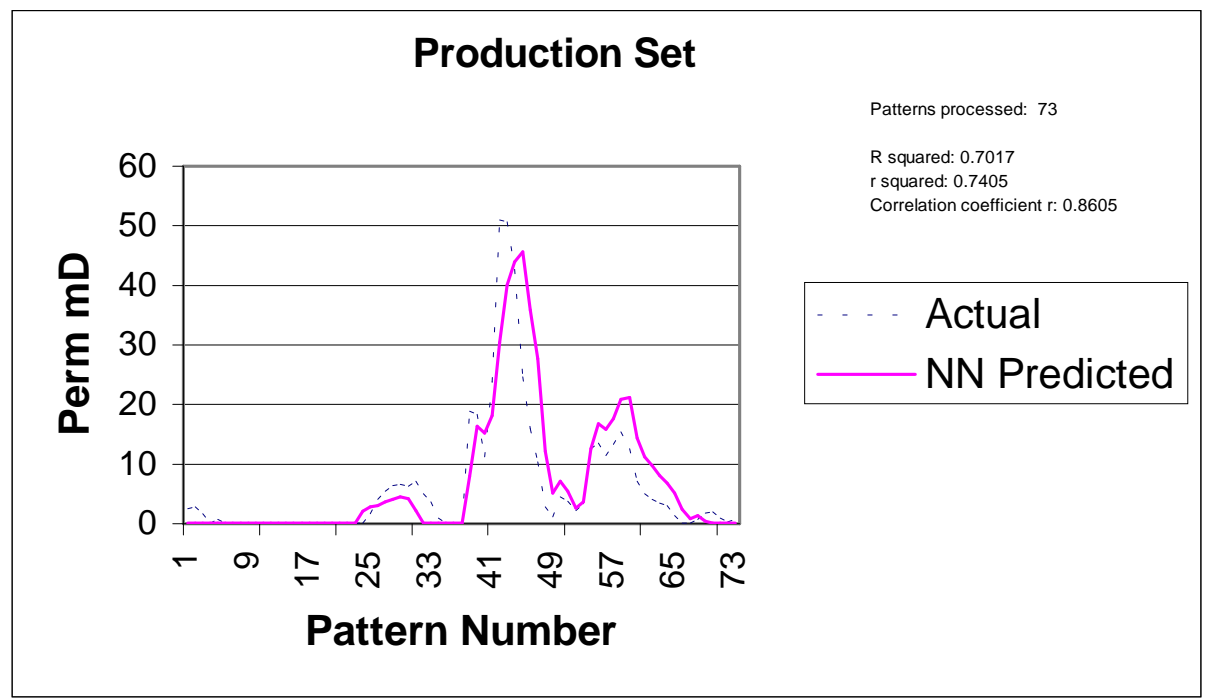

Figure A50. NN Two Verification Net(Cw3), Production Set

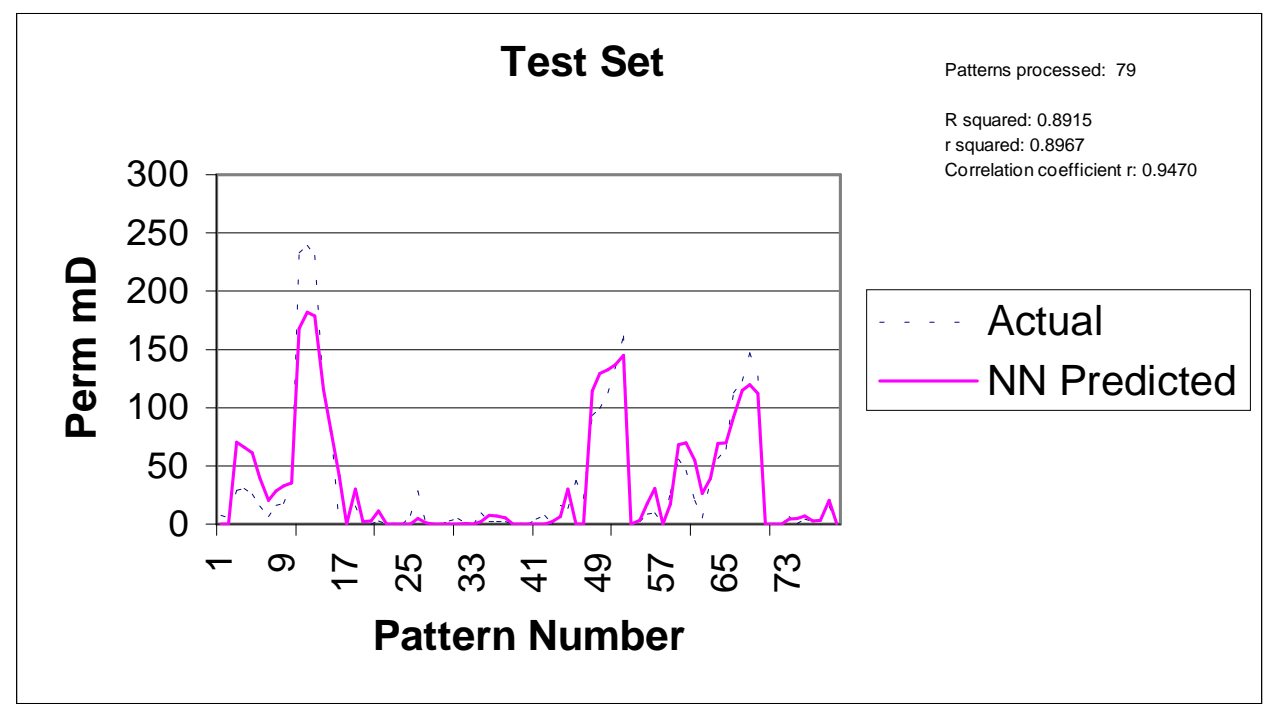

Figure A51. NN Two Verification Net(Cw3), Test Set 


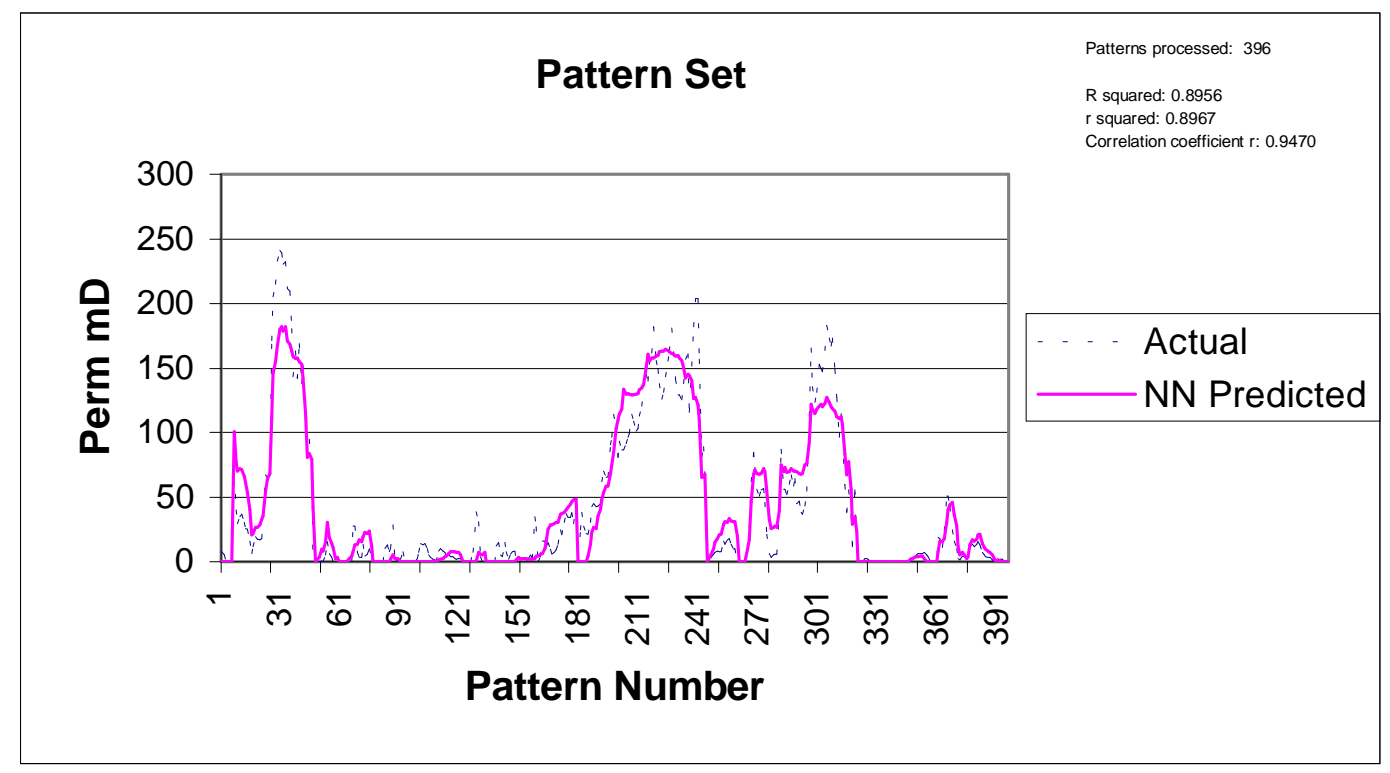

Figure A52. NN Two Verification Net(Cw3), Pattern Set 


\section{Appendix XIII - NN Two Verification Network with Cw4 as Production Set}

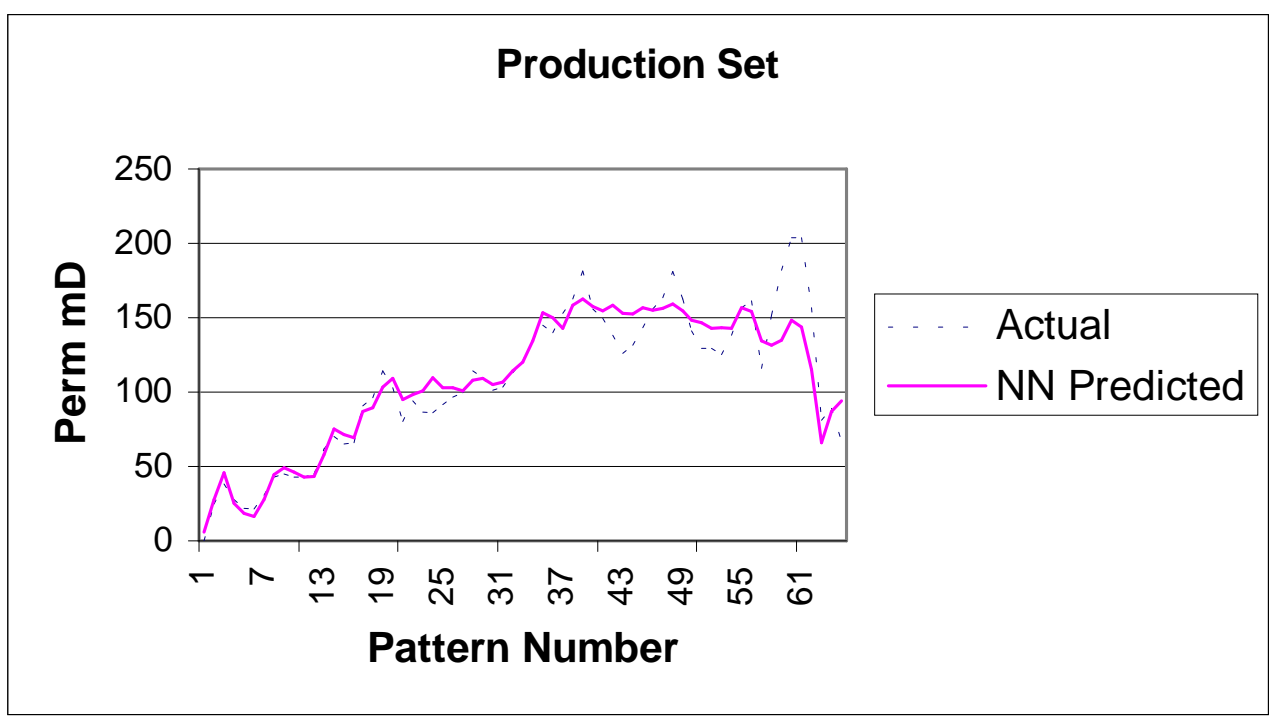

Figure A53. NN Two Verification Net(Cw4), Production Set

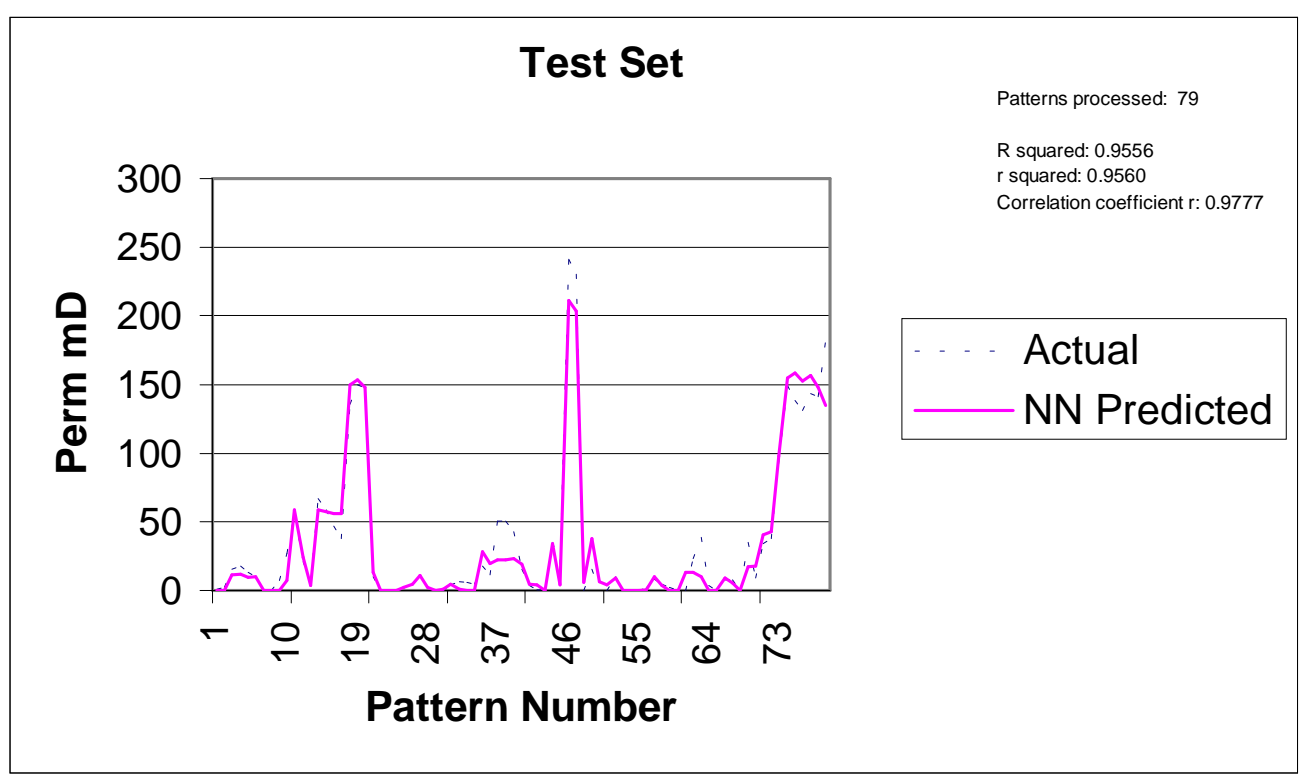

Figure A54. NN Two Verification Net(Cw4), Test Set 


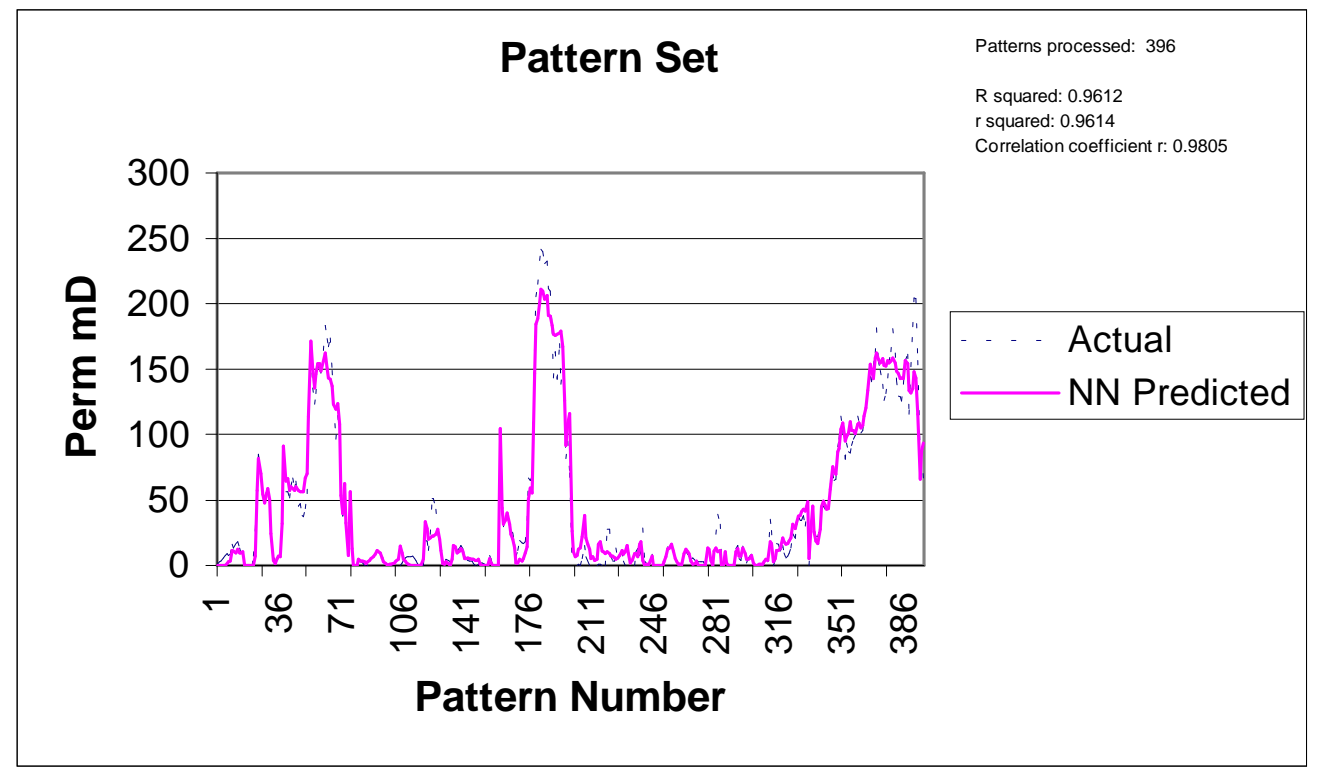

Figure A55. NN Two Verification Net(Cw4), Pattern Set 


\section{Appendix XIV - NN Two Verification Network with Cw5 as Production Set}

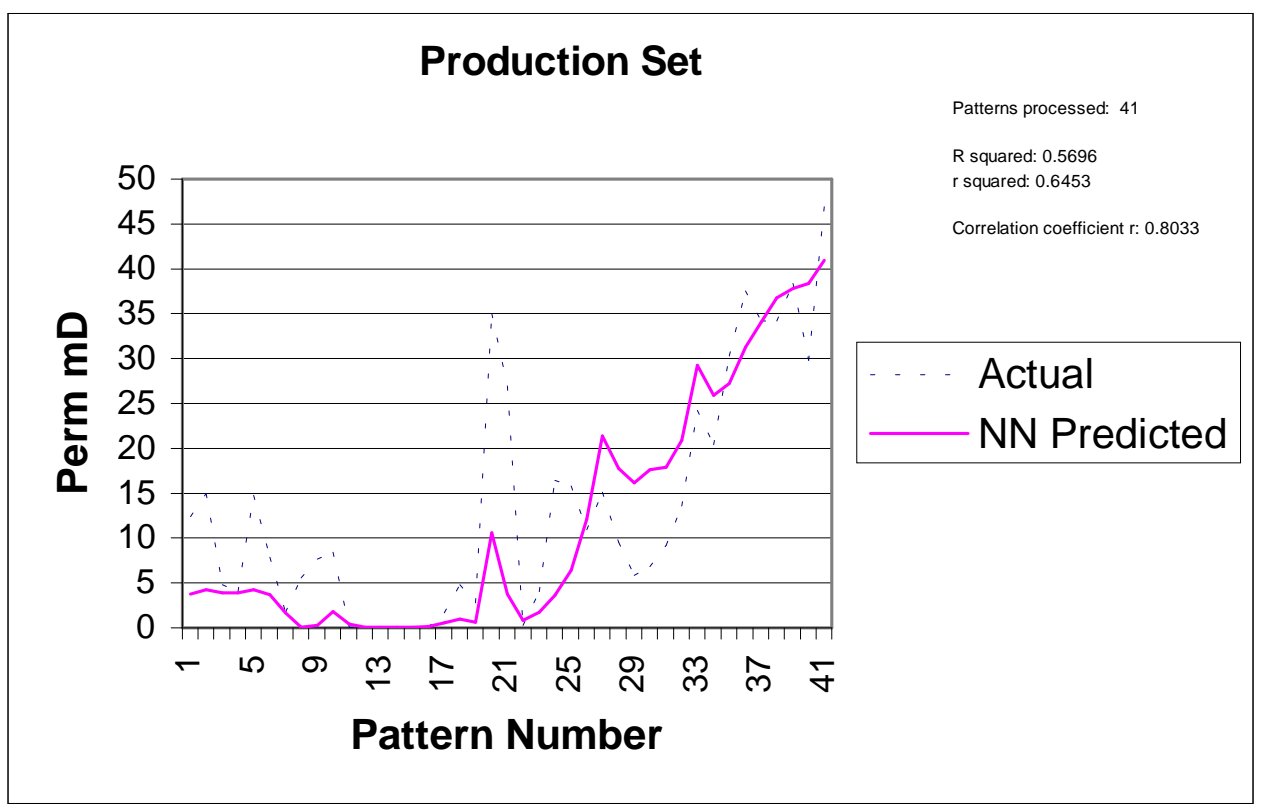

Figure A56. NN Two Verification Net(Cw5), Production Set

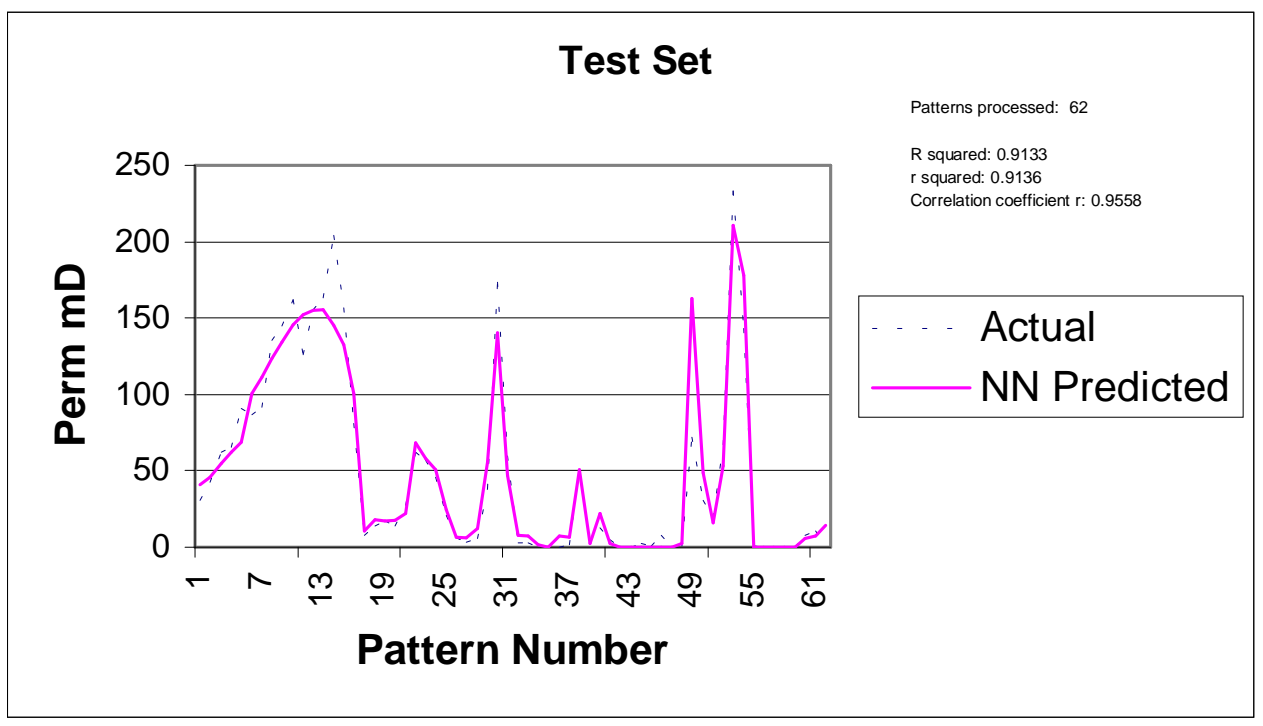

Figure A57. NN Two Verification Net(Cw5), Test Set 


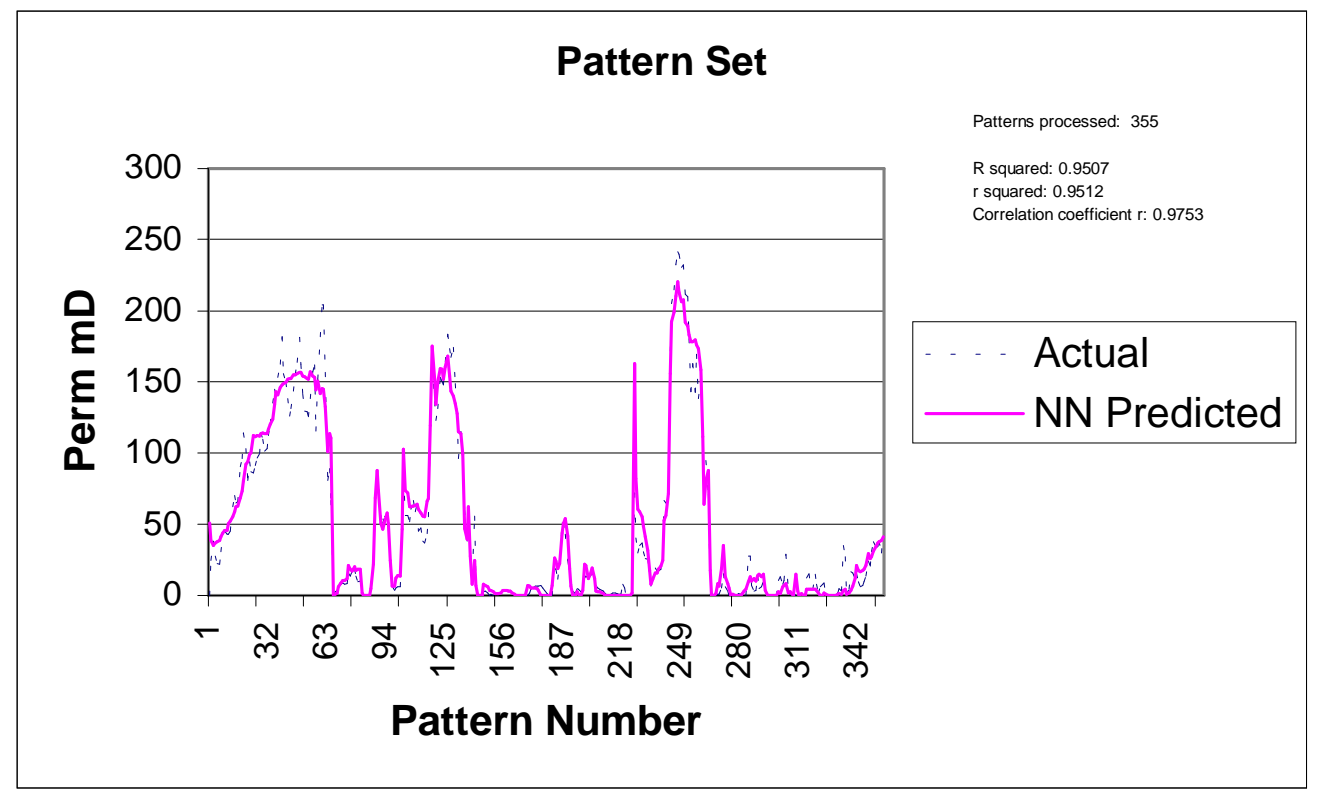

Figure A58. NN Two Verification Net(Cw5), Pattern Set 


\section{Appendix XV - Pilot Area Predictions of Permeability:}

Table A1. Predictions for Pilot Well PI 1

\begin{tabular}{|c|c|}
\hline Depth & NN Predicted Perm mD \\
\hline 2954.75 & 117.94 \\
\hline 2955 & 134.46 \\
\hline 2955.25 & 167.59 \\
\hline 2955.5 & 183.68 \\
\hline 2955.75 & 156.77 \\
\hline 2956 & 166.31 \\
\hline 2956.25 & 162.05 \\
\hline 2956.5 & 159.72 \\
\hline 2956.75 & 152.37 \\
\hline 2957 & 149.76 \\
\hline 2957.25 & 148.51 \\
\hline 2957.5 & 145.24 \\
\hline 2957.75 & 149.48 \\
\hline 2958 & 160.13 \\
\hline 2958.25 & 167.57 \\
\hline 2958.5 & 166.47 \\
\hline 2958.75 & 174.00 \\
\hline 2959 & 158.01 \\
\hline 2959.25 & 146.49 \\
\hline 2959.5 & 146.48 \\
\hline 2959.75 & 145.96 \\
\hline 2960 & 143.13 \\
\hline 2960.25 & 142.48 \\
\hline 2960.5 & 140.19 \\
\hline 2960.75 & 138.79 \\
\hline 2961 & 137.41 \\
\hline 2961.25 & 134.61 \\
\hline 2961.5 & 129.13 \\
\hline 2961.75 & 136.22 \\
\hline 2962 & 141.22 \\
\hline 2962.25 & 147.53 \\
\hline 2962.5 & 81.01 \\
\hline 2962.75 & 119.49 \\
\hline 2963 & 110.82 \\
\hline 2963.25 & 48.71 \\
\hline 2963.5 & 47.25 \\
\hline 2963.75 & 10.20 \\
\hline 2964 & 14.36 \\
\hline 2964.25 & 30.70 \\
\hline 2964.5 & 25.88 \\
\hline 2964.75 & 33.93 \\
\hline 2965 & 41.53 \\
\hline 2965.25 & 81.91 \\
\hline 2965.5 & 53.23 \\
\hline
\end{tabular}


Table A2. Predictions for Pilot Well PI 2

\begin{tabular}{|c|c|}
\hline Depth & $\begin{array}{l}\text { NN Predicted } \\
\text { Perm mD }\end{array}$ \\
\hline 2793.75 & 35.26 \\
\hline 2794 & 27.92 \\
\hline 2794.25 & 17.73 \\
\hline 2794.5 & 19.11 \\
\hline 2794.75 & 23.50 \\
\hline 2795 & 65.91 \\
\hline 2795.25 & 138.39 \\
\hline 2795.5 & 194.48 \\
\hline 2795.75 & 169.24 \\
\hline 2796 & 167.10 \\
\hline 2796.25 & 157.58 \\
\hline 2796.5 & 163.80 \\
\hline 2796.75 & 170.31 \\
\hline 2797 & 148.51 \\
\hline 2797.25 & 122.07 \\
\hline 2797.5 & 113.15 \\
\hline 2797.75 & 105.97 \\
\hline 2798 & 99.20 \\
\hline 2798.25 & 93.38 \\
\hline 2798.5 & 77.61 \\
\hline 2798.75 & 64.30 \\
\hline 2799 & 64.69 \\
\hline 2799.25 & 67.30 \\
\hline 2799.5 & 57.57 \\
\hline 2799.75 & 43.91 \\
\hline 2800 & 49.93 \\
\hline 2800.25 & 89.90 \\
\hline 2800.5 & 80.48 \\
\hline 2800.75 & 40.23 \\
\hline 2801 & 23.46 \\
\hline
\end{tabular}


Table A3. Predictions for Pilot Well PI 3

\begin{tabular}{|c|c|}
\hline Depth & NN Predicted Perm mD \\
\hline 2839.25 & 25.33 \\
\hline 2839.5 & 33.44 \\
\hline 2839.75 & 41.33 \\
\hline 2840 & 51.72 \\
\hline 2840.25 & 88.56 \\
\hline 2840.5 & 95.25 \\
\hline 2840.75 & 79.91 \\
\hline 2841 & 113.42 \\
\hline 2841.25 & 136.49 \\
\hline 2841.5 & 135.29 \\
\hline 2841.75 & 98.03 \\
\hline 2842 & 90.45 \\
\hline 2842.25 & 124.07 \\
\hline 2842.5 & 138.68 \\
\hline 2842.75 & 147.38 \\
\hline 2843 & 161.55 \\
\hline 2843.25 & 163.54 \\
\hline 2843.5 & 150.28 \\
\hline 2843.75 & 138.90 \\
\hline 2844 & 137.40 \\
\hline 2844.25 & 121.70 \\
\hline 2844.5 & 120.28 \\
\hline 2844.75 & 119.07 \\
\hline 2845 & 111.52 \\
\hline 2845.25 & 62.61 \\
\hline 2845.5 & 69.74 \\
\hline 2845.75 & 44.03 \\
\hline & \\
\hline
\end{tabular}


Table A4. Predictions for Pilot Well PI 4

\begin{tabular}{|c|c|}
\hline DEPTH & NN Predicted Perm mD \\
\hline 3082.5 & 4.51 \\
\hline 3082.75 & 8.27 \\
\hline 3083 & 7.15 \\
\hline 3083.25 & 20.14 \\
\hline 3083.5 & 36.72 \\
\hline 3083.75 & 27.49 \\
\hline 3084 & 18.22 \\
\hline 3084.25 & 15.07 \\
\hline 3084.5 & 28.44 \\
\hline 3084.75 & 39.79 \\
\hline 3085 & 28.86 \\
\hline 3085.25 & 11.06 \\
\hline 3085.5 & 5.23 \\
\hline 3085.75 & 8.31 \\
\hline 3086 & 7.22 \\
\hline 3087.75 & 17.45 \\
\hline 3088 & 43.41 \\
\hline 3088.25 & 14.65 \\
\hline 3088.5 & 136.18 \\
\hline 3088.75 & 120.86 \\
\hline 3089 & 88.64 \\
\hline 3089.25 & 120.85 \\
\hline 3089.5 & 124.36 \\
\hline 3089.75 & 104.51 \\
\hline 3090 & 94.30 \\
\hline 3090.25 & 108.94 \\
\hline 3090.5 & 112.62 \\
\hline 3090.75 & 124.35 \\
\hline 3091 & 129.48 \\
\hline 3091.25 & 142.89 \\
\hline 3091.5 & 148.66 \\
\hline 3091.75 & 144.55 \\
\hline 3092 & 137.10 \\
\hline 3092.25 & 90.23 \\
\hline 3092.5 & 81.98 \\
\hline 3092.75 & 75.19 \\
\hline 3093 & 17.77 \\
\hline 3095 & 2.90 \\
\hline 3095.25 & 1.29 \\
\hline 3097.75 & 0.59 \\
\hline 3098.25 & 3.04 \\
\hline 3098.5 & 10.06 \\
\hline 3098.75 & 10.90 \\
\hline 3099 & 11.14 \\
\hline 3099.25 & 6.01 \\
\hline
\end{tabular}


Table A5. Predictions for Pilot Well PI 5

\begin{tabular}{|c|c|}
\hline Depth & NN Predicted Perm mD \\
\hline 2862 & 14.98 \\
\hline 2862.25 & 16.72 \\
\hline 2862.5 & 21.51 \\
\hline 2862.75 & 23.96 \\
\hline 2863 & 25.36 \\
\hline 2863.25 & 23.59 \\
\hline 2863.5 & 25.99 \\
\hline 2863.75 & 26.02 \\
\hline 2864 & 25.05 \\
\hline 2864.25 & 28.26 \\
\hline 2864.5 & 38.73 \\
\hline 2864.75 & 54.78 \\
\hline 2865 & 46.51 \\
\hline 2865.25 & 41.12 \\
\hline 2865.5 & 34.65 \\
\hline 2865.75 & 34.14 \\
\hline 2866 & 45.82 \\
\hline 2866.25 & 46.96 \\
\hline 2866.5 & 53.36 \\
\hline 2866.75 & 49.19 \\
\hline 2867 & 46.43 \\
\hline 2867.25 & 67.57 \\
\hline 2867.5 & 75.37 \\
\hline 2867.75 & 63.48 \\
\hline 2868 & 81.03 \\
\hline 2868.25 & 112.54 \\
\hline 2868.5 & 138.53 \\
\hline 2868.75 & 164.86 \\
\hline 2869 & 185.30 \\
\hline 2869.25 & 218.51 \\
\hline 2869.5 & 203.58 \\
\hline 2869.75 & 208.83 \\
\hline 2870 & 185.74 \\
\hline 2870.25 & 181.35 \\
\hline 2870.5 & 182.94 \\
\hline 2870.75 & 184.25 \\
\hline 2871 & 183.13 \\
\hline 2871.25 & 183.90 \\
\hline 2871.5 & 186.76 \\
\hline 2871.75 & 187.66 \\
\hline 2872 & 189.74 \\
\hline 2872.25 & 196.80 \\
\hline 2872.5 & 196.45 \\
\hline 2872.75 & 196.41 \\
\hline 2873 & 202.32 \\
\hline 2873.25 & 203.55 \\
\hline
\end{tabular}


Permeability Profile for Pilot Well Five Continued

\begin{tabular}{|c|c|}
\hline 2873.5 & 206.20 \\
\hline 2873.75 & 207.31 \\
\hline 2874 & 202.50 \\
\hline 2874.25 & 202.04 \\
\hline 2874.5 & 202.57 \\
\hline 2874.75 & 201.53 \\
\hline 2875 & 197.29 \\
\hline 2875.25 & 196.93 \\
\hline 2875.5 & 198.30 \\
\hline 2875.75 & 195.45 \\
\hline 2876 & 193.23 \\
\hline 2876.25 & 196.25 \\
\hline 2876.5 & 194.33 \\
\hline 2876.75 & 200.81 \\
\hline 2877 & 218.26 \\
\hline 2877.25 & 221.15 \\
\hline 2877.5 & 217.29 \\
\hline 2877.75 & 197.51 \\
\hline 2878 & 163.37 \\
\hline 2878.25 & 165.86 \\
\hline 2878.5 & 161.26 \\
\hline 2878.75 & 134.24 \\
\hline 2879 & 125.07 \\
\hline 2879.25 & 92.20 \\
\hline 2879.5 & 72.88 \\
\hline 2879.75 & 42.02 \\
\hline 2880 & 22.32 \\
\hline 2880.25 & 4.28 \\
\hline 2880.5 & 5.31 \\
\hline
\end{tabular}


Table A6. Predictions for Pilot Well PI 6

\begin{tabular}{|c|c|}
\hline Depth & NN Predicted Perm mD \\
\hline 2943.5 & 16.60 \\
\hline 2943.75 & 9.74 \\
\hline 2944 & 21.82 \\
\hline 2944.25 & 97.65 \\
\hline 2944.5 & 46.20 \\
\hline 2944.75 & 44.44 \\
\hline 2945 & 40.67 \\
\hline 2945.25 & 36.04 \\
\hline 2945.5 & 48.45 \\
\hline 2945.75 & 104.59 \\
\hline 2946 & 109.14 \\
\hline 2946.25 & 87.11 \\
\hline 2946.5 & 84.69 \\
\hline 2946.75 & 83.07 \\
\hline 2947 & 76.31 \\
\hline 2947.25 & 65.66 \\
\hline 2947.5 & 98.05 \\
\hline 2947.75 & 83.76 \\
\hline 2948 & 99.77 \\
\hline 2948.25 & 75.97 \\
\hline 2948.5 & 106.98 \\
\hline 2948.75 & 65.92 \\
\hline 2949 & 56.54 \\
\hline 2949.25 & 61.11 \\
\hline 2949.5 & 61.91 \\
\hline 2949.75 & 92.06 \\
\hline 2950 & 13.99 \\
\hline 2950.25 & 13.80 \\
\hline 2950.5 & 13.86 \\
\hline 2950.75 & 17.64 \\
\hline 2951 & 19.91 \\
\hline 2951.25 & 19.04 \\
\hline
\end{tabular}


Table A7. Predictions for Pilot Well PI 7

\begin{tabular}{|c|c|}
\hline DEPTH & NN Predicted Perm mD \\
\hline 2888.5 & 3.83 \\
\hline 2888.75 & 4.84 \\
\hline 2889 & 6.77 \\
\hline 2889.25 & 8.51 \\
\hline 2889.5 & 26.24 \\
\hline 2889.75 & 28.47 \\
\hline 2890 & 22.80 \\
\hline 2890.25 & 17.20 \\
\hline 2890.5 & 11.66 \\
\hline 2890.75 & 4.46 \\
\hline 2891 & 18.21 \\
\hline 2891.25 & 22.45 \\
\hline 2891.5 & 23.70 \\
\hline 2891.75 & 24.32 \\
\hline 2892 & 40.23 \\
\hline 2892.25 & 47.58 \\
\hline 2892.5 & 47.44 \\
\hline 2892.75 & 57.11 \\
\hline 2893 & 57.38 \\
\hline 2893.25 & 44.40 \\
\hline 2893.5 & 41.72 \\
\hline 2893.75 & 46.16 \\
\hline 2894 & 67.62 \\
\hline 2894.25 & 58.13 \\
\hline 2894.5 & 55.42 \\
\hline 2894.75 & 92.25 \\
\hline 2895 & 98.45 \\
\hline 2895.25 & 113.96 \\
\hline 2895.5 & 93.98 \\
\hline 2895.75 & 109.21 \\
\hline 2896 & 100.93 \\
\hline 2896.25 & 90.67 \\
\hline 2896.5 & 101.72 \\
\hline 2896.75 & 93.76 \\
\hline 2897 & 94.74 \\
\hline 2897.25 & 93.10 \\
\hline 2897.5 & 96.41 \\
\hline 2897.75 & 97.03 \\
\hline 2898 & 95.28 \\
\hline 2898.25 & 96.34 \\
\hline 2898.5 & 102.32 \\
\hline 2898.75 & 107.78 \\
\hline 2899 & 117.30 \\
\hline 2899.25 & 147.83 \\
\hline 2899.5 & 161.92 \\
\hline 2899.75 & 150.26 \\
\hline
\end{tabular}


Permeability Profile for Pilot Well Seven Continued

\begin{tabular}{|c|c|}
\hline 2900 & 166.74 \\
\hline 2900.25 & 169.04 \\
\hline 2900.5 & 159.30 \\
\hline 2900.75 & 156.13 \\
\hline 2901 & 158.01 \\
\hline 2901.25 & 155.56 \\
\hline 2901.5 & 154.88 \\
\hline 2901.75 & 158.42 \\
\hline 2902 & 155.93 \\
\hline 2902.25 & 155.21 \\
\hline 2902.5 & 155.33 \\
\hline 2902.75 & 152.20 \\
\hline 2903 & 146.10 \\
\hline 2903.25 & 144.99 \\
\hline 2903.5 & 139.91 \\
\hline 2903.75 & 138.54 \\
\hline 2904 & 138.83 \\
\hline 2904.25 & 146.22 \\
\hline 2904.5 & 140.68 \\
\hline 2904.75 & 133.70 \\
\hline 2905 & 156.14 \\
\hline 2905.25 & 197.51 \\
\hline 2905.5 & 186.38 \\
\hline 2905.75 & 207.41 \\
\hline 2906 & 131.99 \\
\hline 2906.25 & 38.60 \\
\hline 2906.5 & 76.39 \\
\hline 2906.75 & 86.54 \\
\hline 2907 & 35.11 \\
\hline & \\
\hline
\end{tabular}




\section{References:}

1. Mohaghegh, Ameri, S., Arefi, R., "Virtual Measurement of Heterogeneous Formation Permeability Using Geophysical Well Log Responses", Log Analyst, March-April 1996

2. Molnar, D., Aminian, K., Ameri, S., "The Use of Well Log Data for Estimating Permeability in a Heterogeneous Reservoir", SPE 29175, Proceedings of SPE Eastern Regional Meeting, Charleston, WV, November 1994.

3. Riera, Alexis J., "Predicting Permeability and Flow Capacity Distribution with Back-Propagation Artificial Neural Networks", West Virginia University, Thesis, 2000.

4. Mohaghegh, S., Arefi, R., Bilgesu, H. I., Ameri, S., Rose, D. "Design and Development of an Artificial Neural Network for Estimation of Formation Permeability”, SPE 28237, Proceedings of SPE Petroleum Computer Conference, Dallas, TX, July-August 1994.

5. Ameri, S., Aminian, K., Avary, K. L., Bilgesu, H. I., McDowell, R., Matchen, D., "Reservoir Characterization of Upper Devonian Gordon Sandstone, Stringtown Oilfield, Northwestern West Virginia", WVU and Appalachian Oil and Natural Gas Research Consortium, Semi-Annual Report, October 1999.

6. Aminian, K., "Characterization of a Complex Reservoir in West Virginia", SPE 26939, Proceedings of SPE Eastern Regional Meeting, Pittsburgh, PA, November 1993.

7. Hohn, M., Avary, K. L., McDowell, R., Matchen, D., "Reservoir Characterization of Upper Devonian Gordon Sandstone, Stringtown Oilfield, Northwestern West Virginia", WVU and Appalachian Oil and Natural Gas Research Consortium, Semi-Annual Report, April 1999. 
8. Ameri, S. "Petroleum and Natural Gas Engineering 235 v.1", "Well Log Interpretation", West Virginia University Notebook, 1998.

9. Mohaghegh, S., "Neural Network: What It Can Do for Petroleum Engineers", Journal of Petroleum Technology, January 1995.

10. NeuroShell 2 Help File, Release 4.0, (C) Ward Systems Group, Inc. 1993-1998

11. Kumoluyi, A., Daltaban, T., "Higher-Order Neural Networks in Petroleum Engineering", SPE 27905, Proceedings of SPE Western Regional Meeting, Long Beach, CA, March 1994. 